A modular invariant bulk theory for the $c=\mathbf{0}$ triplet model

This content has been downloaded from IOPscience. Please scroll down to see the full text. 2011 J. Phys. A: Math. Theor. 44015204

(http://iopscience.iop.org/1751-8121/44/1/015204)

View the table of contents for this issue, or go to the journal homepage for more

Download details:

IP Address: 150.203.179.232

This content was downloaded on 08/07/2014 at 00:17

Please note that terms and conditions apply. 


\title{
A modular invariant bulk theory for the $c=0$ triplet model
}

\author{
Matthias R Gaberdiel ${ }^{1}$, Ingo Runkel ${ }^{2}$ and Simon Wood ${ }^{1}$ \\ ${ }^{1}$ Institute for Theoretical Physics, ETH Zürich, 8093 Zürich, Switzerland \\ ${ }^{2}$ Department Mathematik, Universität Hamburg, Bundesstraße 55, 20146 Hamburg, Germany \\ E-mail: gaberdiel@itp.phys.ethz.ch,ingo.runkel@uni-hamburg.de and swood@itp.phys.ethz.ch
}

Received 17 August 2010, in final form 13 October 2010

Published 30 November 2010

Online at stacks.iop.org/JPhysA/44/015204

\begin{abstract}
A proposal for the bulk space of the logarithmic $\mathcal{W}_{2,3}$-triplet model at central charge zero is made. The construction is based on the idea that one may reconstruct the bulk theory of a rational conformal field theory from its boundary theory. The resulting bulk space is a quotient of the direct sum of projective representations, which is isomorphic, as a vector space, to the direct sum of tensor products of the irreducible representations with their projective covers. As a consistency check of our analysis, we show that the partition function of the bulk theory is modular invariant, and that the boundary state analysis is compatible with the proposed annulus partition functions of this model.
\end{abstract}

PACS number: $11.25 . \mathrm{Hf}$

Mathematics Subject Classification: 81T40, 17B68, 81R10

\section{Introduction and summary}

Logarithmic conformal field theories [1] with $c=0$ appear quite generically in models with a non-trivial fixed point theory but a trivial generating function [2]. Prime examples of such theories are systems with quenched disorder [3] that include, among others, percolation [4-10] and polymers [11-14]. Another area where $c=0$ conformal field theories have recently made an appearance is the dual theory for chiral gravity in $\mathrm{AdS}_{3}$ [15], and also in this context there are indications that the conformal field theory is logarithmic [16, 17].

Much has been learned over the last 15 years about logarithmic conformal field theories. In particular, the triplet theory at $c=-2$ [18] has been understood in great detail [19-22], as have been the WZW models with supergroup target spaces [23-28]. There has also been some progress towards understanding WZW models at a fractional level that shows logarithmic behaviour [29-32], logarithmic extensions of minimal models [33] and the structure of general indecomposable Virasoro representations [34]. However, most of the models that have been 
understood behave quite differently from the theory at $c=0$. Indeed, $c=0$ is special since the Virasoro field is 'null' at $c=0$ in the sense that it has a vanishing two-point function. As a consequence the vacuum representation of the chiral algebra is not irreducible, and a number of novel phenomena appear. Many of these are also shared by the whole family of logarithmic minimal $\mathcal{W}_{p, q}$-models with $p, q \geqslant 2$ [35] that have attracted a lot of attention during the last four years [9, 36-46]. Significant progress has been made for these theories, and the fusion rules as well as the modular properties of the chiral representations are now fairly well understood. In [41], a consistent boundary theory was proposed for the simplest member of this family of models, the theory with $c_{2,3}=0$, and the generalization to all $\mathcal{W}_{p, q}$-models was sketched in [43]. However, so far an understanding of the corresponding bulk theory is missing.

In this paper, we shall construct the bulk space of states for the $\mathcal{W}_{2,3}$-model that corresponds to the boundary theory of [41]. Our strategy is to reconstruct the bulk theory, starting from the boundary ansatz. This idea goes back to [47-49] (see also [50] and [51, 52]), and was successfully applied to the logarithmic $\mathcal{W}_{1, p}$-triplet models (and in particular to the theory at $c=-2$ ) in [53]. There are a number of subtleties that arise in the present context, and as a consequence the analysis is technically considerably harder than in [53]. However, the resulting space of bulk states still has all properties one should expect it to have. In particular, the partition function is modular invariant, and it allows for the construction of boundary states in agreement with the prediction of [41]. Both of these are non-trivial consistency checks, and we are therefore confident that our bulk ansatz defines a consistent bulk conformal field theory.

Many structural features are similar to what happens for the $\mathcal{W}_{1, p}$-models (and in particular at $c=-2$ ). For example, the space $\mathcal{H}_{\text {bulk }}$ of bulk states is of the form

$$
\mathcal{H}_{\text {bulk }}=\bigoplus_{i \in \text { Irr }} \mathcal{W}(i) \otimes_{\mathbb{C}} \overline{\mathcal{P}}(i) \quad \text { as }\left(L_{0}^{\text {diag }}, \bar{L}_{0}^{\text {diag }}\right) \text {-graded vector space }
$$

where the sum runs over all irreducible $\mathcal{W}_{2,3}$-representations $\mathcal{W}(i), \mathcal{P}(i)$ denotes the projective cover of $\mathcal{W}(i)$, and $L_{0}^{\text {diag }}$ (resp. $\bar{L}_{0}^{\text {diag }}$ ) refers to the diagonal part of the action of $L_{0}$ (resp. $\bar{L}_{0}$ ). However, there are also a number of remarkable new phenomena that appear for the $\mathcal{W}_{2,3}$-model at $c=0$. Firstly, in distinction to all rational CFTs and the $\mathcal{W}_{1, p}$-models, the bulk theory does not contain $\mathcal{W}_{2,3} \otimes_{\mathbb{C}} \overline{\mathcal{W}}_{2,3}$ as a sub-representation, but only as a sub-quotient. The second surprising feature is that in order to reproduce the annulus amplitudes one only needs ten Ishibashi states, while the characters of the representations labelling the boundary states of [41] span a 12-dimensional space. The actual boundary states must involve additional Ishibashi states in order for the bulk-boundary correlators to be non-degenerate in the bulk entry, but these additional Ishibashi states do not contribute to the annulus diagrams (without any additional insertions), and are hence invisible from the point of view of the usual annulus analysis.

The paper is organized as follows. In the remainder of the introduction section, we shall first review some of the results from [41] that we shall need in the rest of the paper. In particular, section 1.1 reviews the representation theory of the $\mathcal{W}_{2,3}$-algebra in question, while section 1.2 summarizes the boundary theory that was proposed in [41]. In section 1.3, we sketch the general strategy for the construction of the bulk theory starting from this boundary ansatz. Finally, section 1.4 describes the main features of the corresponding boundary state analysis. These sections are meant to give a non-technical account of these considerations; the actual details are then spelled out in sections 2 and 3, respectively, while section 4 contains our conclusions. Some of the tables, diagrams and technical constructions have been delegated into appendices. 


\subsection{The chiral $\mathcal{W}_{2,3}$-model}

The $\mathcal{W}_{p, q}$-models have central charge

$$
c_{p, q}=1-6 \frac{(p-q)^{2}}{p q},
$$

where $p$ and $q$ are a pair of positive co-prime integers. In the following, we shall only consider the case $(p, q)=(2,3)$ for which $c_{2,3}=0$. The chiral algebra $\mathcal{W} \equiv \mathcal{W}_{2,3}$ is generated by the Virasoro algebra, as well as by a triplet of fields of conformal weight $h=15$. In the vacuum representation $L_{-1} \Omega=0$, but $T=L_{-2} \Omega \neq 0$. Since the positive modes annihilate $T$ (in particular, $c=0$ implies that $L_{2} T=0$ ), the vacuum representation contains a proper sub-representation, namely the highest weight representation $\mathcal{W}(2)$ generated from $T$ by the action of the negative modes. Thus, $\mathcal{W}$ is not irreducible, but has the schematic structure

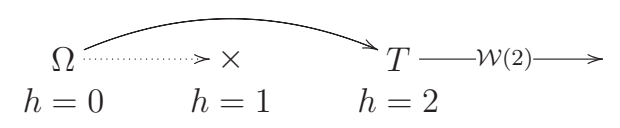

The irreducible representations of the $\mathcal{W}$ algebra are described by the finite Kac table [35]:

\begin{tabular}{c|ccc} 
& $s=1$ & $s=2$ & $s=3$ \\
\hline$r=1$ & $0,2,7$ & $0,1,5$ & $\frac{1}{3}, \frac{10}{3}$ \\
$r=2$ & $\frac{5}{8}, \frac{33}{8}$ & $\frac{1}{8}, \frac{21}{8}$ & $\frac{-1}{24}, \frac{35}{24}$
\end{tabular}

Here each entry $h$ is the conformal dimension of the highest weight states of an irreducible representation, which we shall denote by $\mathcal{W}(h)$. There is only one representation corresponding to $h=0$, namely the one-dimensional vacuum representation $\mathcal{W}(0)$, spanned by the vacuum vector $\Omega$. The significance of the grey boxes will be explained below in section 1.2.

As is familiar from other logarithmic theories, the 13 irreducible representations in (1.4) do not close among themselves under fusion. The minimal set of representations which includes (1.4) and is closed under fusion and taking conjugates involves in addition the 22 indecomposable representations

$$
\begin{aligned}
& \mathcal{W}, \mathcal{W}^{*}, \mathcal{Q}, \mathcal{Q}^{*}, \quad \mathcal{R}^{(2)}(0,2)_{7}, \quad \mathcal{R}^{(2)}(2,7), \quad \mathcal{R}^{(2)}(0,1)_{5}, \quad \mathcal{R}^{(2)}(1,5), \\
& \mathcal{R}^{(2)}(0,2)_{5}, \quad \mathcal{R}^{(2)}(2,5), \quad \mathcal{R}^{(2)}(0,1)_{7}, \quad \mathcal{R}^{(2)}(1,7), \quad \mathcal{R}^{(2)}\left(\frac{1}{3}, \frac{1}{3}\right), \\
& \mathcal{R}^{(2)}\left(\frac{1}{3}, \frac{10}{3}\right), \quad \mathcal{R}^{(2)}\left(\frac{5}{8}, \frac{5}{8}\right), \quad \mathcal{R}^{(2)}\left(\frac{5}{8}, \frac{21}{8}\right), \quad \mathcal{R}^{(2)}\left(\frac{1}{8}, \frac{1}{8}\right), \quad \mathcal{R}^{(2)}\left(\frac{1}{8}, \frac{33}{8}\right), \\
& \mathcal{R}^{(3)}(0,0,1,1), \quad \mathcal{R}^{(3)}(0,0,2,2), \quad \mathcal{R}^{(3)}(0,1,2,5), \quad \mathcal{R}^{(3)}(0,1,2,7),
\end{aligned}
$$

whose structure (including characters and embedding diagrams) is described in some detail in [41, appendix A] and A below. Note that in addition to the chiral algebra $\mathcal{W}$ also its conjugate representation $\mathcal{W}^{*}$ appears; because of the structure of (1.3) it is not isomorphic to $\mathcal{W}$. (Indeed, the embedding structure of $\mathcal{W}^{*}$ can be obtained from that of $\mathcal{W}$ by reversing the direction of the arrow $\Omega \rightarrow T$.)

The associative fusion rules of all of these $13+22=35$ representations were given explicitly in [41]. They were derived based on a direct calculation of the fusion rules for the Virasoro subalgebra (compare [36]) and agree with what was obtained based on a lattice analysis in $[9,40]$. 
For the construction of the bulk theory, the projective covers of the irreducible representations play an important role. Let us denote the projective cover of the representation $\mathcal{W}(h)$ by $\mathcal{P}(h)$. Then we have the identifications (this was already suggested in [41])

$$
\begin{array}{lll}
\mathcal{P}(1)=\mathcal{R}^{(3)}(0,0,1,1), & \mathcal{P}(2)=\mathcal{R}^{(3)}(0,0,2,2), & \mathcal{P}(5)=\mathcal{R}^{(3)}(0,1,2,5), \\
\mathcal{P}(7)=\mathcal{R}^{(3)}(0,1,2,7), & \mathcal{P}\left(\frac{1}{3}\right)=\mathcal{R}^{(2)}\left(\frac{1}{3}, \frac{1}{3}\right), & \mathcal{P}\left(\frac{10}{3}\right)=\mathcal{R}^{(2)}\left(\frac{1}{3}, \frac{10}{3}\right), \\
\mathcal{P}\left(\frac{5}{8}\right)=\mathcal{R}^{(2)}\left(\frac{5}{8}, \frac{5}{8}\right), & \mathcal{P}\left(\frac{21}{8}\right)=\mathcal{R}^{(2)}\left(\frac{5}{8}, \frac{21}{8}\right), & \mathcal{P}\left(\frac{1}{8}\right)=\mathcal{R}^{(2)}\left(\frac{1}{8}, \frac{1}{8}\right), \\
\mathcal{P}\left(\frac{33}{8}\right)=\mathcal{R}^{(2)}\left(\frac{1}{8}, \frac{33}{8}\right), & \mathcal{P}\left(\frac{-1}{24}\right)=\mathcal{W}\left(\frac{-1}{24}\right), & \mathcal{P}\left(\frac{35}{24}\right)=\mathcal{W}\left(\frac{35}{24}\right) .
\end{array}
$$

As was also explained in [41], none of the representations in (1.4) and (1.5) can be the projective cover of $\mathcal{W}(0)$. On the other hand, on general grounds [54-56] one may expect that every irreducible representation has a projective cover. We shall propose the structure of the projective cover $\mathcal{P}(0)$ for $\mathcal{W}(0)$ in section 2.2.2 below. In appendix $\mathrm{B}$, we shall derive some further properties of $\mathcal{P}(0)$ and deduce its fusion rules with most of the representations in (1.4) and (1.5).

\subsection{The boundary theory}

For non-logarithmic rational conformal field theories with charge-conjugation modular invariant (the 'Cardy case'), the boundary conditions that preserve the chiral symmetry are in one-to-one correspondence with the representations of the chiral algebra [57]. The situation is similar for the $\mathcal{W}_{1, p}$-models, but as was explained in [41], the situation is more complicated for the $\mathcal{W}_{2,3}$-model at $c=0$. Indeed, consistent boundary conditions can only be associated with a subset of the 35 representations above, namely to those 26 representations that are not in grey boxes ${ }^{3}$. These allowed representations are characterized by the property that the conjugate representation agrees with the dual representation. This condition guarantees that the space of boundary fields has a non-degenerate bilinear pairing, i.e. that the boundary two-point functions are non-degenerate (for more details see [41]). In particular, this then also implies that the space of boundary fields of any consistent boundary condition cannot just be $\mathcal{W}_{2,3}$ itself.

The space of open string states $\mathcal{H}_{\mathcal{R} \rightarrow \mathcal{S}}$ between two boundary conditions labelled by $\mathcal{R}$ and $\mathcal{S}$ is given by the fusion product $\mathcal{H}_{\mathcal{R} \rightarrow \mathcal{S}}=\mathcal{S} \otimes_{f} \mathcal{R}^{*}$, where $\otimes_{f}$ denotes the fusion product of $\mathcal{W}$-representations and $\mathcal{R}^{*}$ is the conjugate representation to $\mathcal{R}$; this is as in the usual Cardy case.

A convenient tool to analyse the annulus partition functions, i.e. the characters of the $\mathcal{W}$-representation $\mathcal{H}_{\mathcal{R} \rightarrow \mathcal{S}}$, is provided by the (additive) Grothendieck group $\mathrm{K}_{0}$. It consists of equivalence classes [R] of representations $\mathcal{R}$, where two representations $\mathcal{R}$ and $\mathcal{R}^{\prime}$ are equivalent iff they have the same $\operatorname{character}^{4}, \chi_{\mathcal{R}}(q)=\chi_{\mathcal{R}^{\prime}}(q)$. Thus, giving the element $[\mathcal{R}]$ in $\mathrm{K}_{0}$ for a representation $\mathcal{R}$ is the same as specifying its character, and we can describe the annulus partition functions as elements $\left[\mathcal{H}_{\mathcal{R} \rightarrow \mathcal{S}}\right] \in \mathrm{K}_{0}$.

The additive group $\mathrm{K}_{0}$ is generated by 13 elements, corresponding to the characters of the irreducible representations. The representations which lead to consistent boundary conditions, i.e. those not in the grey boxes, generate a subgroup $\mathrm{K}_{0}^{b}$ of $\mathrm{K}_{0}$ spanned (for example) by the following 12 independent elements [41, section 2.4]:

$$
\begin{aligned}
\mathrm{K}_{0}^{b}= & \operatorname{span}_{\mathbb{Z}}\left([\mathcal{W}(h)] \mid h=0, \frac{1}{3}, \frac{10}{3}, \frac{5}{8}, \frac{33}{8}, \frac{1}{8}, \frac{21}{8}, \frac{-1}{24}, \frac{35}{24}\right) \\
& \oplus 2 \mathbb{Z}([\mathcal{W}(2)]+[\mathcal{W}(7)]) \oplus 2 \mathbb{Z}([\mathcal{W}(1)]+[\mathcal{W}(5)]) \oplus 2 \mathbb{Z}([\mathcal{W}(2)]+[\mathcal{W}(5)]) .
\end{aligned}
$$

\footnotetext{
3 There may, however, be additional boundary conditions associated to other classes of representations.

4 This description of $\mathrm{K}_{0}$ is correct if the irreducible representations of $\mathcal{W}$ have linearly independent characters, which is the case for $\mathcal{W}_{2,3}$.
} 
The fusion product makes $\mathrm{K}_{0}^{b}$ (but not $\mathrm{K}_{0}$ ) into a ring. By definition, this means that the annulus partition function for the boundary conditions $\mathcal{R}$ and $\mathcal{S}$ can be written as

$$
\left[\mathcal{H}_{\mathcal{R} \rightarrow \mathcal{S}}\right]=[\mathcal{S}] \cdot\left[\mathcal{R}^{*}\right] .
$$

Clearly, two representations $\mathcal{R}$ and $\mathcal{R}^{\prime}$ lead to the same annulus partition function $\left[\mathcal{H}_{\mathcal{R} \rightarrow \mathcal{S}}\right]=$ $\left[\mathcal{H}_{\mathcal{R}^{\prime} \rightarrow \mathcal{S}}\right]$ if they have the same character, because then $[\mathcal{R}]=\left[\mathcal{R}^{\prime}\right]$. However, the converse is not true: the ring $\mathrm{K}_{0}^{b}$ contains a two-dimensional null ideal spanned by

$N_{1}=\left[\mathcal{W}\left(\frac{5}{8}\right)\right]-\left[\mathcal{W}\left(\frac{33}{8}\right)\right]-\left[\mathcal{W}\left(\frac{1}{8}\right)\right]+\left[\mathcal{W}\left(\frac{21}{8}\right)\right]-\left[\mathcal{W}\left(\frac{-1}{24}\right)\right]+\left[\mathcal{W}\left(\frac{35}{24}\right)\right], N_{2}=[\mathcal{W}(0)]$

These two elements have the property that $N_{1} \cdot C=0=N_{2} \cdot C$ for all $C \in \mathrm{K}_{0}^{b}$. Thus, any two representations $\mathcal{R}$ and $\mathcal{R}^{\prime}$ with the property that $[\mathcal{R}]-\left[\mathcal{R}^{\prime}\right] \in \operatorname{span}_{\mathbb{Z}}\left(\left[N_{1}\right],\left[N_{2}\right]\right)$ also lead to identical annulus partition functions. For example, the two representations $\mathcal{R}=\mathcal{W}\left(\frac{5}{8}\right) \oplus \mathcal{W}\left(\frac{21}{8}\right) \oplus \mathcal{W}\left(\frac{35}{24}\right)$ and $\mathcal{R}^{\prime}=\mathcal{W}\left(\frac{33}{8}\right) \oplus \mathcal{W}\left(\frac{1}{8}\right) \oplus \mathcal{W}\left(\frac{-1}{24}\right)$ have the property that $[\mathcal{R}]-\left[\mathcal{R}^{\prime}\right]=\left[N_{1}\right]$. As a consequence, their annulus amplitudes with any boundary condition $\mathcal{S}$ agree, $\left[\mathcal{H}_{\mathcal{R} \rightarrow \mathcal{S}}\right]=\left[\mathcal{H}_{\mathcal{R}^{\prime} \rightarrow \mathcal{S}}\right]$. This does not imply that $\mathcal{R}$ and $\mathcal{R}^{\prime}$ have identical boundary states $\| \mathcal{R}\rangle\rangle$ and $\left.\left.\| \mathcal{R}^{\prime}\right\rangle\right\rangle$; indeed, one may expect that suitable correlators of bulk fields on a disc can distinguish two boundary conditions $\mathcal{R}$ and $\mathcal{R}^{\prime}$ whenever $[\mathcal{R}] \neq\left[\mathcal{R}^{\prime}\right]$ in $\mathrm{K}_{0}^{b}$. However, it does mean that ten Ishibashi states are sufficient to reproduce all annulus partition functions as overlaps $\left\langle\left\langle\mathcal{R}\left\|q^{L_{0}} \bar{q}^{\bar{L}_{0}}\right\| \mathcal{S}\right\rangle\right\rangle$.

\subsection{From boundary to bulk}

For non-logarithmic rational conformal field theories, one can reconstruct the bulk theory from one consistent boundary condition $[48,49]$. The same method was also successfully applied in [53] to the case of the logarithmic $\mathcal{W}_{1, p}$-models. The idea behind the construction is as follows.

Suppose we are given a boundary condition together with its space of boundary fields and the associated operator product expansion (OPE). We consider the disc correlator involving one boundary field and one field from the yet-to-be-constructed space of bulk states. This correlator defines a pairing between the bulk and boundary degrees of freedom

$$
\mathcal{H}_{\text {bulk }}^{(\text {ans })} \times \mathcal{H}_{\text {bnd }} \rightarrow \mathbb{C}:(\psi, \phi) \mapsto\langle V(\psi, 0) V(\phi, u)\rangle_{\text {disc }},
$$

where $|u|=1$ is a point on the perimeter of the disc, and the index '(ans)' indicates that at this stage we can only make an ansatz for the bulk space of states. If $\phi \in \mathcal{R} \otimes_{\mathbb{C}} \overline{\mathcal{S}}$ is in a representation of the left- and right-moving chiral algebra, then the map (1.10) is uniquely determined up to some constants (one for each allowed fusion channel) since, by the usual doubling trick, the correlator can be thought of as a chiral three-point function. These constants encode the bulk-boundary OPE, and they are constrained by two necessary requirements. First of all, it follows from general principles (non-degeneracy of the bulk twopoint function on the sphere) that the map (1.10) has to be non-degenerate in the first (bulk) entry. Furthermore, given the OPE of the boundary fields, it also defines correlators involving more than one boundary field, and these have to satisfy the appropriate locality conditions, see section 2.1.2. The 'correct' space of bulk states is then simply the largest possible space compatible with these requirements. It was proven in [51, 52] that in the non-logarithmic setting, this construction reproduces indeed the unique space of bulk states that is compatible with the given boundary condition. For the logarithmic $\mathcal{W}_{1, p}$-models, the construction was also shown to lead to sensible results; in particular, the known $c=-2$ bulk theory of [20] was correctly reproduced by this method in [53]. 
In all of these constructions, the analysis is simplest if the space of boundary fields just consists of the chiral algebra (or VOA) itself. This is the case for the 'identity Cardy brane', and it leads to the charge-conjugation modular invariant theory. Indeed, such a brane exists for all the $\mathcal{W}_{1, p}$-models, and it was taken as the starting point for the analysis of [53]. As already noted at the beginning of section 1.2, one of the complications for $c=0$ is that such a boundary condition does not exist since the non-degeneracy of the boundary two-point function forbids the space of boundary fields to consist just of the chiral algebra itself. However, as we shall propose in section 2.2, for the purpose of analysing the consistency of the bulk ansatz, one may assume that the space of boundary fields just consists of $\mathcal{W}^{*}$, the conjugate of the VOA $\mathcal{W}$, so that we can proceed very similar to [53]. In particular, the solution to the maximality condition is

$$
\mathcal{H}_{\text {bulk }}=\left(\bigoplus_{j} \mathcal{P}(j) \otimes_{\mathbb{C}} \overline{\mathcal{P}}(j)^{*}\right) / \mathcal{N},
$$

where the sum runs over all irreducible representations, $\mathcal{P}(j)$ denotes the corresponding projective cover, and $\mathcal{N}$ is a certain subspace that can be calculated as in [53], and that guarantees that the bulk-boundary map is non-degenerate in the bulk entry. For the $\mathcal{W}_{2,3^{-}}$ model, we will see in section 2.2 that the space of bulk states has the form

$$
\mathcal{H}_{\text {bulk }}=\mathcal{H}_{0} \oplus \mathcal{H}_{\frac{1}{8}} \oplus \mathcal{H}_{\frac{5}{8}} \oplus \mathcal{H}_{\frac{1}{3}} \oplus \mathcal{H}_{\frac{-1}{24}} \oplus \mathcal{H}_{\frac{35}{24}},
$$

where we have labelled the individual blocks $\mathcal{H}_{h}$ by the conformal weight of the lowest state. The corresponding characters are explicitly given by

$$
\begin{aligned}
& \operatorname{tr}_{\mathcal{H}_{0}}\left(q^{L_{0}} \bar{q}^{\bar{L}_{0}}\right)= 2\left|\chi_{\mathcal{W}(0)}(q)\right|^{2}+\mid \chi_{\mathcal{W}(0)}(q)+2 \chi_{\mathcal{W}(1)}(q)+2 \chi_{\mathcal{W}(2)}(q) \\
&+2 \chi_{\mathcal{W}(5)}(q)+\left.2 \chi_{\mathcal{W}(7)}(q)\right|^{2}, \\
& \operatorname{tr}_{\mathcal{H}_{\frac{1}{8}}}\left(q^{L_{0}} \bar{q}^{L_{0}}\right)= 2\left|\chi_{\mathcal{W}\left(\frac{1}{8}\right)}(q)+\chi_{\mathcal{W}\left(\frac{33}{8}\right)}(q)\right|^{2}, \\
& \operatorname{tr}_{\mathcal{H}_{\frac{5}{8}}}\left(q^{L_{0}} \bar{q}^{L_{0}}\right)= 2\left|\chi_{\mathcal{W}\left(\frac{5}{8}\right)}(q)+\chi_{\mathcal{W}\left(\frac{21}{8}\right)}(q)\right|^{2}, \\
& \operatorname{tr}_{\mathcal{H}_{\frac{1}{3}}}\left(q^{L_{0}} \bar{q}^{\bar{L}_{0}}\right)= 2\left|\chi_{\mathcal{W}\left(\frac{1}{3}\right)}(q)+\chi_{\mathcal{W}\left(\frac{10}{3}\right)}(q)\right|^{2}, \\
& \operatorname{tr}_{\mathcal{H}_{\frac{-1}{24}}}\left(q^{L_{0}} \bar{q}^{L_{0}}\right)=\left|\chi_{\mathcal{W}\left(-\frac{1}{24}\right)}(q)\right|^{2}, \quad \text { and } \quad \operatorname{tr}_{\mathcal{H}_{\frac{35}{24}}}\left(q^{L_{0}} \bar{q}^{\bar{L}_{0}}\right)=\left|\chi_{\mathcal{W}\left(\frac{35}{24}\right)}(q)\right|^{2},
\end{aligned}
$$

where $\chi_{\mathcal{W}(h)}(q)$ denotes the character of the irreducible representation $\mathcal{W}(h)$; see [35] or [41, appendix A.1] for explicit expressions. Our construction, however, contains much more information than just these characters; in fact, our analysis leads to a description of the $\mathcal{H}_{h}$ as a representation of $\mathcal{W} \otimes_{\mathbb{C}} \overline{\mathcal{W}}$.

A convenient way to represent the structure of these (not fully reducible) representations is the composition series, which is defined as follows. Starting from a representation $M_{1}$, one finds the largest sub-representation $R_{1}$ which can be written as a direct sum of irreducible representations - these are called composition factors. Then one takes the quotient of $M_{1}$ by $R_{1}$ and repeats the procedure with $M_{2}=M_{1} / R_{1}$. In other words, one constructs a chain of sub-representations

$$
M_{1}=A_{n} \supset A_{n-1} \supset \cdots \supset A_{2} \supset A_{1}=R_{1}
$$

such that $R_{i}=A_{i} / A_{i-1}$ is a direct sum of irreducible representations ${ }^{5}$. We represent the quotients $R_{i}$ of a composition series as

$$
R_{n} \rightarrow R_{n-1} \rightarrow \cdots \rightarrow R_{2} \rightarrow R_{1}
$$

5 It is more common to require each quotient $A_{i} / A_{i-1}$ to be irreducible, rather than fully reducible. In this case, the composition series is only unique up to permutations of its composition factors. 
The action of $\mathcal{W} \otimes_{\mathbb{C}} \overline{\mathcal{W}}$ either maps states within a representation $R_{j}$ into one another, or moves them along arrows in the composition series.

With this technology at hand, we can now describe the composition series of the representations appearing in (1.12). For $\mathcal{H}_{\frac{-1}{24}}$ and $\mathcal{H}_{\frac{35}{24}}$, the composition series consists just of a single term

$$
\mathcal{H}_{\frac{-1}{24}}: \mathcal{W}\left(\frac{-1}{24}\right) \otimes_{\mathbb{C}} \overline{\mathcal{W}}\left(\frac{-1}{24}\right), \quad \mathcal{H}_{\frac{35}{24}}: \mathcal{W}\left(\frac{35}{24}\right) \otimes_{\mathbb{C}} \overline{\mathcal{W}}\left(\frac{35}{24}\right)
$$

For $\mathcal{H}_{\frac{1}{8}}$ it has the same structure as in the $\mathcal{W}_{1, p}$-models (see [28] and [53])

$$
\begin{array}{rlll}
\mathcal{W}\left(\frac{1}{8}\right) \otimes_{\mathbb{C}} \overline{\mathcal{W}}\left(\frac{1}{8}\right) & \oplus \mathcal{W}\left(\frac{33}{8}\right) \otimes_{\mathbb{C}} \overline{\mathcal{W}}\left(\frac{33}{8}\right) \\
\mathcal{H}_{\frac{1}{8}}: & 2 \cdot \mathcal{W}\left(\frac{1}{8}\right) \otimes_{\mathbb{C}} \overline{\mathcal{W}}\left(\frac{33}{8}\right) & \oplus & 2 \cdot \mathcal{W}\left(\frac{33}{8}\right) \otimes_{\mathbb{C}} \overline{\mathcal{W}}\left(\frac{1}{8}\right) \\
\mathcal{W}\left(\frac{1}{8}\right) \otimes_{\mathbb{C}} \overline{\mathcal{W}}\left(\frac{1}{8}\right) & \oplus & \mathcal{W}\left(\frac{33}{8}\right) \otimes_{\mathbb{C}} \overline{\mathcal{W}}\left(\frac{33}{8}\right),
\end{array}
$$

where we have written the composition series vertically. This is easier to visualize if we represent each direct sum by a little table where we indicate the multiplicity of each term $\mathcal{W}(h) \otimes_{\mathbb{C}} \overline{\mathcal{W}}(\bar{h})$. For example, the composition series for $\mathcal{H}_{1 / 8}$ is then written as

\begin{tabular}{c|c|c} 
& $\frac{1}{8}$ & $\frac{33}{8}$ \\
\hline$\frac{1}{8}$ & 1 & 0 \\
\hline$\frac{33}{8}$ & 0 & 1 \\
\hline
\end{tabular}

\begin{tabular}{c|c|c|} 
& $\frac{1}{8}$ & $\frac{33}{8}$ \\
\hline$\frac{1}{8}$ & 0 & 2 \\
\hline$\frac{33}{8}$ & 2 & 0
\end{tabular}$\longrightarrow$\begin{tabular}{c|c|c|} 
& $\frac{1}{8}$ & $\frac{33}{8}$ \\
\hline$\frac{1}{8}$ & 1 & 0 \\
\hline$\frac{33}{8}$ & 0 & 1 \\
\hline
\end{tabular}.

Here the horizontal direction gives $h$ and the vertical direction $\bar{h}$. The picture for $\mathcal{H}_{5 / 8}$ and $\mathcal{H}_{1 / 3}$ looks the same, with $\left\{\frac{1}{8}, \frac{33}{8}\right\}$ replaced by $\left\{\frac{5}{8}, \frac{21}{8}\right\}$ and $\left\{\frac{1}{3}, \frac{10}{3}\right\}$, respectively. For $\mathcal{H}_{0}$, the composition series is more complicated,

\begin{tabular}{l|l|l|l|l|l|l|} 
& 0 & 1 & 2 & 5 & 7 & \\
\hline 0 & 1 & & & & & \\
\hline 1 & & 1 & & & & \\
\hline 2 & & & 1 & & & \\
\hline 5 & & & & 1 & & \\
\hline 7 & & & & & 1 \\
\hline
\end{tabular}

\begin{tabular}{l|l|l|l|l|l|} 
& 0 & 1 & 2 & 5 & 7 \\
\hline 0 & & 1 & 1 & & \\
\hline 1 & 1 & & & 2 & 2 \\
\hline 2 & 1 & & & 2 & 2 \\
\hline 5 & & 2 & 2 & & \\
\hline 7 & & 2 & 2 & & \\
\hline
\end{tabular}$\longrightarrow$

\begin{tabular}{l|l|l|l|l|l|} 
& 0 & 1 & 2 & 5 & 7 \\
\hline 0 & 1 & & & 2 & 2 \\
\hline 1 & & 2 & 4 & & \\
\hline 2 & & 4 & 2 & & \\
\hline 5 & 2 & & & 2 & 4 \\
\hline 7 & 2 & & & 4 & 2 \\
\hline \multicolumn{7}{|c}{$\mid$}
\end{tabular}

\begin{tabular}{c|c|c|c|c|c|} 
& 0 & 1 & 2 & 5 & 7 \\
\hline 0 & & 1 & 1 & & \\
\hline 1 & 1 & & & 2 & 2 \\
\hline 2 & 1 & & & 2 & 2 \\
\hline 5 & & 2 & 2 & & \\
\hline 7 & & 2 & 2 & &
\end{tabular}

All empty entries are equal to ' 0 '.

Adding all the tables in a composition series reproduces the multiplicities given in the partition function for each $\mathcal{H}_{h}$ from (1.13). The complete partition function turns out to be modular invariant, as must be the case for a consistent conformal field theory. This represents a non-trivial consistency check on our analysis. 
It is also worth mentioning that a non-degenerate bulk two-point function requires that $\mathcal{H}_{\text {bulk }}$ is isomorphic to its conjugate representation $\mathcal{H}_{\text {bulk }}^{*}$. A necessary condition for this is that the composition series does not change when reversing all arrows, which indeed holds for the series given above and provides another consistency check of our construction.

\subsection{The boundary states}

With the detailed knowledge of the proposed bulk theory at hand, we can study whether the boundary conditions of [41] can actually be described in terms of appropriate boundary states. More specifically, we can ask whether we can reproduce the annulus partition functions of [41] in terms of suitable boundary states of our proposed bulk theory.

The first step of this analysis can be done without any detailed knowledge of the bulk theory. The proposal of [41] for the boundary conditions makes a prediction for the various annulus partition functions. Because of the two-dimensional null ideal, see equation (1.9), we expect that these annulus amplitudes can be reproduced by boundary states that are linear combinations of 10 (rather than 12) Ishibashi states. This turns out to be correct: if we label the necessary Ishibashi states in the various sectors of the bulk space (1.12) as

$\left.\left.\mathcal{H}_{\frac{-1}{24}}, \mathcal{H}_{\frac{35}{24}}:\left|\frac{-1}{24}\right\rangle\right\rangle, \quad\left|\frac{35}{24}\right\rangle\right\rangle$,

$\left.\left.\left.\left.\left.\left.\mathcal{H}_{\frac{1}{8}}, \mathcal{H}_{\frac{5}{8}}, \mathcal{H}_{\frac{1}{3}}:\left|\frac{1}{8}, A\right\rangle\right\rangle,\left|\frac{1}{8}, B\right\rangle\right\rangle,\left|\frac{5}{8}, A\right\rangle\right\rangle,\left|\frac{5}{8}, B\right\rangle\right\rangle,\left|\frac{1}{3}, A\right\rangle\right\rangle,\left|\frac{1}{3}, B\right\rangle\right\rangle$,

$\left.\mathcal{H}_{0}:|0,+\rangle\right\rangle,|0,-\rangle$,

and assume - this question will be addressed momentarily — that their overlaps can be chosen to be (with $q=\mathrm{e}^{2 \pi \mathrm{i} \tau}$ )

$\left\langle\left\langle\frac{-1}{24}\left|q^{L_{0}+\bar{L}_{0}}\right| \frac{-1}{24}\right\rangle\right\rangle=\sqrt{3} \chi_{\mathcal{W}\left(\frac{-1}{24}\right)}(q)$

$\left\langle\left\langle\frac{35}{24}\left|q^{L_{0}+\bar{L}_{0}}\right| \frac{35}{24}\right\rangle\right\rangle=-\sqrt{3} \chi_{\mathcal{W}\left(\frac{35}{24}\right)}(q)$

$\left\langle\left\langle\frac{1}{8}, A\left|q^{L_{0}+\bar{L}_{0}}\right| \frac{1}{8}, A\right\rangle\right\rangle=-\frac{\mathrm{i} \tau}{3}\left(\chi_{\mathcal{W}\left(\frac{1}{8}\right)}(q)-2 \chi_{\mathcal{W}\left(\frac{33}{8}\right)}(q)\right)$

$\left\langle\left\langle\frac{1}{8}, A\left|q^{L_{0}+\bar{L}_{0}}\right| \frac{1}{8}, B\right\rangle\right\rangle=\frac{2}{\sqrt{3}}\left(\chi_{\mathcal{W}\left(\frac{33}{8}\right)}(q)+\chi_{\mathcal{W}\left(\frac{1}{8}\right)}(q)\right)$

$\left\langle\left\langle\frac{5}{8}, A\left|q^{L_{0}+\bar{L}_{0}}\right| \frac{5}{8}, A\right\rangle\right\rangle=-\frac{\mathrm{i} \tau 2}{3}\left(\chi_{\mathcal{W}\left(\frac{5}{8}\right)}(q)-\frac{1}{2} \chi_{\mathcal{W}\left(\frac{21}{8}\right)}(q)\right)$

$\left\langle\left\langle\frac{5}{8}, A\left|q^{L_{0}+\bar{L}_{0}}\right| \frac{5}{8}, B\right\rangle\right\rangle=\frac{2}{\sqrt{3}}\left(\chi_{\mathcal{W}\left(\frac{21}{8}\right)}(q)+\chi_{\mathcal{W}\left(\frac{5}{8}\right)}(q)\right)$

$\left\langle\left\langle\frac{1}{3}, A\left|q^{L_{0}+\bar{L}_{0}}\right| \frac{1}{3}, A\right\rangle=\mathrm{i} \tau 2 \sqrt{3}\left(\chi_{\mathcal{W}\left(\frac{1}{3}\right)}(q)-\chi_{\mathcal{W}\left(\frac{10}{3}\right)}(q)\right)\right.$

$\left\langle\left\langle\frac{1}{3}, A\left|q^{L_{0}+\bar{L}_{0}}\right| \frac{1}{3}, B\right\rangle\right\rangle=\frac{2}{\sqrt{3}}\left(\chi_{\mathcal{W}\left(\frac{1}{3}\right)}(q)+\chi_{\mathcal{W}\left(\frac{10}{3}\right)}(q)\right)$

$\left\langle\left\langle 0, \pm\left|q^{L_{0}+\bar{L}_{0}}\right| 0, \pm\right\rangle\right\rangle=\frac{1}{2} \chi_{\mathcal{W}(0)}(q)=\frac{1}{2}$

$\left\langle\left\langle 0, \pm\left|q^{L_{0}+\bar{L}_{0}}\right| 0, \mp\right\rangle\right\rangle=\frac{1}{2}\left(\chi_{\mathcal{W}(0)}(q)+2 \chi_{\mathcal{W}(1)}(q)+2 \chi_{\mathcal{W}(2)}(q)+2 \chi_{\mathcal{W}(5)}(q)+2 \chi_{\mathcal{W}(7)}(q)\right)$,

the annulus amplitudes are reproduced by

$$
\begin{aligned}
& \left.\left.\left.\left.\left.\left.\left.\| \mathcal{W}\left(-\frac{1}{24}\right)\right\rangle\right\rangle=\left|\frac{-1}{24}\right\rangle\right\rangle-\left|\frac{35}{24}\right\rangle\right\rangle+3\left|\frac{1}{3}, B\right\rangle\right\rangle+\left|\frac{5}{8}, B\right\rangle\right\rangle-\left|\frac{1}{8}, B\right\rangle\right\rangle \\
& \left.\left.\left.\left.\left.\left.\left.\| \mathcal{W}\left(\frac{35}{24}\right)\right\rangle\right\rangle=\left|\frac{-1}{24}\right\rangle\right\rangle-\left|\frac{35}{24}\right\rangle\right\rangle-3\left|\frac{1}{3}, B\right\rangle\right\rangle+\left|\frac{5}{8}, B\right\rangle\right\rangle-\left|\frac{1}{8}, B\right\rangle\right\rangle \\
& \left.\left.\left.\left.\left.\left.\left.\left.\| \mathcal{W}\left(\frac{1}{3}\right)\right\rangle\right\rangle=\frac{1}{2}\left[\left|\frac{-1}{24}\right\rangle\right\rangle+\left|\frac{35}{24}\right\rangle\right\rangle\right]+\frac{1}{2}\left|\frac{1}{3}, A\right\rangle\right\rangle+\frac{1}{2}\left[\left|\frac{5}{8}, B\right\rangle\right\rangle+\left|\frac{1}{8}, B\right\rangle\right\rangle\right]-\frac{2}{\sqrt{3}}|0+\rangle\right\rangle
\end{aligned}
$$




$$
\begin{aligned}
& \left.\left.\left.\left.\left.\left.\left.\left.\| \mathcal{W}\left(\frac{10}{3}\right)\right\rangle\right\rangle=\frac{1}{2}\left[\left|\frac{-1}{24}\right\rangle\right\rangle+\left|\frac{35}{24}\right|\right\rangle\right]-\frac{1}{2}\left|\frac{1}{3}, A\right\rangle\right\rangle+\frac{1}{2}\left[\left|\frac{5}{8}, B\right\rangle\right\rangle+\left|\frac{1}{8}, B\right|\right\rangle\right]+\frac{2}{\sqrt{3}}|0+\rangle\right\rangle \\
& \left.\left.\left.\left.\left.\left.\left.\left.\left.\| \mathcal{W}\left(\frac{5}{8}\right)\right\rangle\right\rangle=\frac{1}{3}\left[\left|\frac{-1}{24}\right\rangle\right\rangle-\left|\frac{35}{24}\right\rangle\right\rangle\right]+\left|\frac{1}{3}, B\right\rangle\right\rangle+\left|\frac{5}{8}, A\right\rangle\right\rangle+\left|\frac{1}{8}, A\right\rangle\right\rangle-\frac{1}{12}\left[\left|\frac{5}{8}, B\right\rangle\right\rangle-\left|\frac{1}{8}, B\right\rangle \mid\right]-|0-\rangle\right\rangle \\
& \left.\left.\left.\left.\left.\left.\left.\left.\left.\| \mathcal{W}\left(\frac{33}{8}\right)\right\rangle\right\rangle=\frac{1}{3}\left[\left|\frac{-1}{24}\right\rangle\right\rangle-\left|\frac{35}{24}\right\rangle\right\rangle\right]-\left|\frac{1}{3}, B\right\rangle\right\rangle+\left|\frac{5}{8}, A\right\rangle\right\rangle+\left|\frac{1}{8}, A\right\rangle\right\rangle-\frac{1}{12}\left[\left|\frac{5}{8}, B\right\rangle\right\rangle-\left|\frac{1}{8}, B\right\rangle \mid\right]+|0-\rangle\right\rangle \\
& \left.\left.\left.\left.\left.\left.\left.\left.\left.\| \mathcal{W}\left(\frac{1}{8}\right)\right\rangle\right\rangle=\frac{2}{3}\left[\left|\frac{-1}{24}\right\rangle\right\rangle-\left|\frac{35}{24}\right\rangle\right\rangle\right]-2\left|\frac{1}{3}, B\right\rangle\right\rangle-\left|\frac{5}{8}, A\right\rangle\right\rangle-\left|\frac{1}{8}, A\right\rangle\right\rangle-\frac{5}{12}\left[\left|\frac{5}{8}, B\right\rangle\right\rangle-\left|\frac{1}{8}, B\right\rangle \mid\right]-|0-\rangle\right\rangle \\
& \left.\left.\left.\left.\left.\left.\left.\left.\left.\| \mathcal{W}\left(\frac{21}{8}\right)\right\rangle\right\rangle=\frac{2}{3}\left[\left|\frac{-1}{24}\right\rangle\right\rangle-\left|\frac{35}{24}\right\rangle\right\rangle\right]+2\left|\frac{1}{3}, B\right\rangle\right\rangle-\left|\frac{5}{8}, A\right\rangle\right\rangle-\left|\frac{1}{8}, A\right\rangle\right\rangle-\frac{5}{12}\left[\left|\frac{5}{8}, B\right\rangle\right\rangle-\left|\frac{1}{8}, B\right\rangle \mid\right]+|0-\rangle\right\rangle \\
& \left.\left.\left.\left.\left.\left.\left.\| R^{(2)}(0,2)_{7}\right\rangle\right\rangle=\frac{2}{3}\left[\left|\frac{-1}{24}\right\rangle\right\rangle+\left|\frac{35}{24}\right\rangle \mid\right]+2\left[\left|\frac{5}{8}, A\right\rangle\right\rangle-\left|\frac{1}{8}, A\right\rangle\right\rangle\right]-\frac{1}{6}\left[\left|\frac{5}{8}, B\right\rangle\right\rangle+\left|\frac{1}{8}, B\right\rangle\right\rangle\right] \\
& \left.\left.\left.\left.\| \mathcal{R}^{(2)}(2,7)\right\rangle\right\rangle=\| R^{(2)}(0,2)_{7}\right\rangle\right\rangle \\
& \left.\left.\left.\left.\left.\left.\left.\left.\| \mathcal{R}^{(2)}(1,5)\right\rangle\right\rangle=\frac{4}{3}\left[\left|\frac{-1}{24}\right\rangle\right\rangle+\left|\frac{35}{24}\right|\right\rangle\right]-2\left[\left|\frac{5}{8}, A\right\rangle\right\rangle-\left|\frac{1}{8}, A\right|\right\rangle\right]-\frac{5}{6}\left[\left|\frac{5}{8}, B\right\rangle\right\rangle+\left|\frac{1}{8}, B\right\rangle\right\rangle\right] \\
& \left.\left.\left.\left.\left.\left.\left.\left.\| \mathcal{R}^{(2)}(2,5)\right\rangle\right\rangle=\left|\frac{-1}{24}\right\rangle\right\rangle+\left|\frac{35}{24}\right|\right\rangle+\left|\frac{1}{3}, A\right\rangle\right\rangle-\frac{1}{2}\left[\left|\frac{5}{8}, B\right\rangle\right\rangle+\left|\frac{1}{8}, B\right\rangle\right\rangle\right]+\frac{2}{\sqrt{3}}|0+\rangle\right\rangle \text {. }
\end{aligned}
$$

This solution was found using the same technique as in [53, section 5.2], i.e. by recursively constructing the boundary states using the knowledge of the annulus partition functions. The $\mathcal{W}_{2,3}$-representations corresponding to the above boundary states generate all of $\mathrm{K}_{0}^{b}$ (see [41, section 2.4]); thus the boundary state $\| \mathcal{R}\rangle$ for an arbitrary representation in $\mathrm{K}_{0}^{b}$ is a linear combination of the above states with integral coefficients. We should also stress that in the above boundary states we have only included components that have a non-zero overlap with at least one other boundary state- these are the only ones that can be detected in annulus amplitudes. The full boundary state must contain additional contributions, see section 3.3.3.

Up to now we have not used any knowledge about the bulk spectrum of the theory. Next, we want to check whether the above Ishibashi states (1.20) (out of which our boundary states were formed) actually exist within our bulk space $\mathcal{H}_{\text {bulk }}$. It is not difficult to classify all Ishibashi states following [53], and we find

$$
\begin{aligned}
& \mathcal{H}_{\frac{-1}{24}}, \mathcal{H}_{\frac{35}{24}}: 1 \text { Ishibashi state each } \\
& \mathcal{H}_{\frac{1}{8}}, \mathcal{H}_{\frac{5}{8}}, \mathcal{H}_{\frac{1}{3}}: 3 \text { Ishibashi states each } \\
& \mathcal{H}_{0}: 9 \text { Ishibashi states. }
\end{aligned}
$$

The Ishibashi states (1.20) can be expressed in terms of linear combinations of the general Ishibashi states in (1.23). Furthermore, the structure of the bulk theory constrains the form of the corresponding overlaps, and this is perfectly compatible with (1.21). This is a highly non-trivial consistency check of our analysis.

\section{The construction of the bulk space}

\subsection{From boundary to bulk-the original construction}

Let us begin by reviewing how the original construction for the space of bulk fields starting from a given boundary condition works. This construction was developed for rational conformal field theories in [47-49,51], and then successfully applied to the logarithmic $\mathcal{W}_{1, p}$-models in $[22,53]$ (for the logarithmic analogue of the 'charge-conjugation modular invariant', i.e. the Cardy case).

The discussion in sections 2.1.1-2.2.1 is generic and does not rely on $\mathcal{W}=\mathcal{W}_{2,3}$. On the other hand, the arguments from section 2.2.2 onwards are specific to $\mathcal{W}=\mathcal{W}_{2,3}$.

2.1.1. The algebra of boundary fields. We want to explain more precisely what we mean by a consistent boundary theory. First, we recall that for each $\mathcal{W}$-representation $U$ there exists a conjugate representation $U^{*}$ that is defined on the graded dual of $U$ [54, definition 2.35]. 
The operation of taking the conjugate of a representation defines a contravariant functor from $\operatorname{Rep}(\mathcal{W})$ to itself which we denote by $(-)^{*}$.

With this definition, we can then explain what we mean by a $\mathcal{W}$-symmetric algebra of boundary fields (or boundary algebra for short): it is a collection of data $\left(\mathcal{H}_{\mathrm{bnd}}, m, \eta, \varepsilon\right)$ where (see also [53, section 1.2])

(1) $\mathcal{H}_{\text {bnd }}$ is the space of boundary fields and forms a $\mathcal{W}$-representation.

(2) $m \in \operatorname{Hom}\left(\mathcal{H}_{\text {bnd }} \otimes_{f} \mathcal{H}_{\text {bnd }}, \mathcal{H}_{\text {bnd }}\right)$ is an intertwiner describing the associative boundary $\mathrm{OPE}^{6}$. That is, if we denote the corresponding intertwining operator by $V_{m}$ : $\mathcal{H}_{\text {bnd }} \otimes_{\mathbb{C}} \mathcal{H}_{\text {bnd }} \rightarrow \mathcal{H}_{\text {bnd }}$, for each $\psi \in \mathcal{H}_{\text {bnd }}$ we obtain a linear map $V_{m}(\psi, x)$ from $\mathcal{H}_{\text {bnd }}$ to (a completion of) $\mathcal{H}_{\text {bnd }}$, and the OPE $\psi(x) \psi^{\prime}(0)$ of two boundary fields $\psi, \psi^{\prime} \in \mathcal{H}_{\text {bnd }}$ is written as $V_{m}(\psi, x) \psi^{\prime}$.

(3) $\eta: \mathcal{W} \rightarrow \mathcal{H}_{\text {bnd }}$ is an injective $\mathcal{W}$-intertwiner such that $\eta(\Omega)$ is the identity field in $\mathcal{H}_{\text {bnd }}$. Since $\eta$ is injective, the entire VOA $\mathcal{W}$ is contained in $\mathcal{H}_{\text {bnd }}$, as is required for a $\mathcal{W}$-symmetric boundary condition.

(4) $\varepsilon: \mathcal{H}_{\text {bnd }} \rightarrow \mathcal{W}^{*}$ is a $\mathcal{W}$-intertwiner describing one-point functions of boundary fields. It has to give rise to a non-degenerate and symmetric two-point function on the boundary.

We will sometimes just write $\mathcal{H}_{\mathrm{bnd}}$ in place of $\left(\mathcal{H}_{\mathrm{bnd}}, m, \eta, \varepsilon\right)$. Given a boundary algebra, the $n$-point function of boundary fields $\psi_{1}, \ldots, \psi_{n} \in \mathcal{H}_{\text {bnd }}$ on the upper half plane is given by

$$
\left\langle\psi_{1}\left(x_{1}\right) \cdots \psi_{n}\left(x_{n}\right)\right\rangle_{\mathrm{uhp}}=\left\langle\varepsilon\left(V_{m}\left(\psi_{1}, x_{1}\right) \cdots V_{m}\left(\psi_{n-1}, x_{n-1}\right) \psi_{n}\right), \Omega\right\rangle,
$$

where we take $x_{n}=0$ and $x_{1}>x_{2}>\ldots>x_{n}$.

2.1.2. Bulk-boundary maps. For the rational theories and the $\mathcal{W}_{1, p}$-models, one could define the bulk space as a solution to a universal property. In order to formulate this construction, we need the notion of a bulk-boundary map.

Fix a boundary algebra $\mathcal{H}_{\text {bnd }}$ and let $\mathcal{H}$ be a $\mathcal{W} \otimes_{\mathbb{C}} \overline{\mathcal{W}}$-representation. By a bulk-boundary map, we mean a linear map $\beta(-, y): \mathcal{H} \rightarrow \mathcal{H}_{\text {bnd }}$ (here $y>0$, and we again suppress the algebraic completion of the target from the notation) which satisfies the following two conditions.

(i) $\beta$ is compatible with the $\mathcal{W}$-symmetry. Given $\phi \in \mathcal{H}$, the element $\beta(\phi, y)$ is to be thought of as an insertion of the bulk field $\phi$ on the upper half plane at position iy, expanded in terms of boundary fields at position 0 . The compatibility condition is formulated as follows. Write $\mathcal{H}$ as a quotient $\mathcal{H}=\hat{\mathcal{H}} / Q$, where $\hat{\mathcal{H}}=\bigoplus_{a} M_{a} \otimes_{\mathbb{C}} \bar{N}_{a}$ for some index set $a$ and $Q$ is a sub-representation of $\hat{\mathcal{H}}$. Denote by $\beta_{a}$ the composition

$$
\beta_{a}(-, y)=M_{a} \otimes_{\mathbb{C}} \bar{N}_{a} \hookrightarrow \hat{\mathcal{H}} \rightarrow \mathcal{H} \stackrel{\beta(-, y)}{\longrightarrow} \mathcal{H}_{\text {bnd }}
$$

Then we require that

$$
\beta_{a}\left(\phi \otimes_{\mathbb{C}} \phi^{\prime}, y\right)=V_{l}(\phi, \mathrm{i} y) V_{r}\left(\phi^{\prime},-\mathrm{i} y\right) \Omega,
$$

for appropriate intertwining operators $V_{r}(-, x): N_{a} \times \mathcal{W} \rightarrow N_{a}$ and $V_{l}(-, x)$ : $M_{a} \times N_{a} \rightarrow \mathcal{H}_{\text {bnd }}$. Here, $V_{r}(-, x) \Omega$ can be taken to be just translation by $x$. In particular, the space of all maps $\beta_{a}$ is isomorphic to $\operatorname{Hom}\left(M_{a} \otimes_{f} N_{a}, \mathcal{H}_{\text {bnd }}\right)$. We denote by $\tilde{\beta}_{a} \in \operatorname{Hom}\left(M_{a} \otimes_{f} N_{a}, \mathcal{H}_{\mathrm{bnd}}\right)$ the image of $\beta_{a}$ under that isomorphism.

${ }^{6}$ Here, $\otimes_{f}$ denotes the fusion product. By construction of $\otimes_{f}$, for three $\mathcal{W}$-representations $R, S$, and $U$, the vector space of intertwining operators $V(-, x): R \otimes_{\mathbb{C}} S \rightarrow U$ (we suppress the formal variable and algebraic completion in the notation of the target vector space) is canonically isomorphic to the space of $\mathcal{W}$-intertwiners $\operatorname{Hom}\left(R \otimes_{f} S, U\right)$, see [54, definition 3.18 and proposition 4.15$]$. 
(ii) $\beta$ is central. Since $\beta(\phi, y)$ corresponds to a bulk field $\phi$ inserted at iy, an $n$-point correlator of boundary fields involving $\beta(\phi, y)$ should be continuous when a boundary field is taken past zero. This gives rise to the centrality condition: write $\mathcal{H}=\hat{\mathcal{H}} / Q$ as in (a). The map $\beta(-, y)$ is called central iff for all $\psi \in \mathcal{H}_{\text {bnd }}$ :

$$
\lim _{x \searrow 0} V_{m}(\psi, x) \beta(\phi, y)=\lim _{x \searrow 0} V_{m}(\beta(\phi, y), x) \psi .
$$

This can also be expressed in terms of the braiding $c_{U, V}: U \otimes_{f} V \rightarrow V \otimes_{f} U$ in the category of $\mathcal{W}$-representations, using the component maps $\tilde{\beta}_{a}$ introduced in (a),

$$
m \circ\left(\operatorname{id}_{\mathcal{H}_{\text {bnd }}} \otimes_{f} \tilde{\beta}_{a}\right) \circ\left(c_{M_{a}, \mathcal{H}_{\text {bnd }}} \otimes_{f} \operatorname{id}_{N_{a}}\right)=m \circ\left(\tilde{\beta}_{a} \otimes_{f} \operatorname{id}_{\mathcal{H}_{\text {bnd }}}\right) \circ\left(\operatorname{id}_{M_{a}} \otimes_{f} c_{\mathcal{H}_{\text {bnd }}, N_{a}}\right) .
$$

A graphical representation of condition (2.5) is ${ }^{7}$.

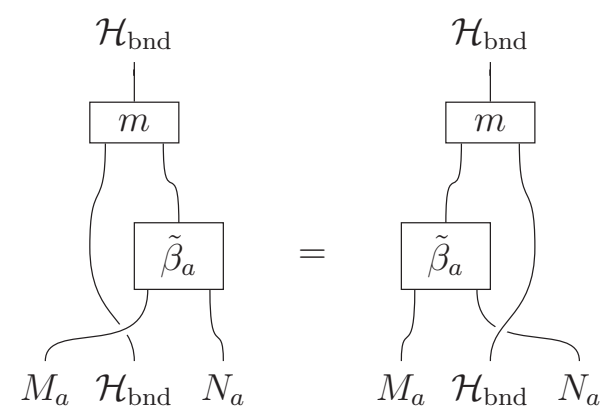

We will sometimes also refer to $\tilde{\beta}_{a}$ as a 'bulk-boundary map'. In the non-logarithmic rational case, bulk-boundary maps were first systematically studied in $[59,60]$; the centrality condition amounts to [60, figure $9(d)]$. In the framework of vertex operator algebras and intertwining operators, the bulk-boundary map and its categorical expression (2.5) have appeared in [61, sections 1.8 and 3.2].

2.1.3. Characterizing the bulk space. After these definitions, we can now explain how the bulk space of states could be characterized for the rational theories [47-49, 51], and for the $\mathcal{W}_{1, p}$-models in the case of the 'charge-conjugation modular invariant' $[22,53]$, the logarithmic analogue of the Cardy case.

Let us fix a boundary algebra $\mathcal{H}_{\text {bnd }}$. We consider pairs $(\mathcal{H}, \beta)$, where $\mathcal{H}$ is a $\mathcal{W} \otimes_{\mathbb{C}} \overline{\mathcal{W}}$-representation and $\beta: \mathcal{H} \rightarrow \mathcal{H}_{\text {bnd }}$ is a bulk-boundary map. By an arrow $f:(\mathcal{H}, \beta) \rightarrow\left(\mathcal{H}^{\prime}, \beta^{\prime}\right)$ between two such pairs we mean a $\mathcal{W} \otimes_{\mathbb{C}} \overline{\mathcal{W}}$-intertwiner $f: \mathcal{H} \rightarrow \mathcal{H}^{\prime}$ such that the diagram

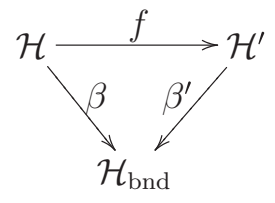

commutes. The space of bulk fields $\mathcal{H}_{\mathrm{bulk}}$ and the bulk-boundary OPE $\beta_{\mathrm{bb}}(-, y)$ for the boundary algebra $\mathcal{H}_{\text {bnd }}$ are then defined to be a terminal object in the category formed by

7 Here we use the usual graphical notation of braided tensor categories [58]; our diagrams are read from bottom to top. 
these pairs and arrows. In other words, the pair $\left(\mathcal{H}_{\text {bulk }}, \beta_{\text {bb }}\right)$ has the property that for all other pairs $\left(\mathcal{H}^{\prime}, \beta^{\prime}\right)$ there exists a unique $\mathcal{W} \otimes_{\mathbb{C}} \overline{\mathcal{W}}$-intertwiner $f: \mathcal{H}^{\prime} \rightarrow \mathcal{H}_{\text {bulk }}$ such that we have $\beta^{\prime}=\beta_{\mathrm{bb}} \circ f: \mathcal{H}^{\prime} \rightarrow \mathcal{H}_{\text {bnd }}$.

A terminal object need not exist, but if it does, it is unique up to unique isomorphism. Indeed, for another terminal object $\left(\mathcal{H}_{\text {bulk }}^{\prime}, \beta_{\text {bb }}^{\prime}\right)$, there are unique intertwiners $\mathcal{H}_{\text {bulk }} \rightarrow \mathcal{H}_{\text {bulk }}^{\prime}$ and $\mathcal{H}_{\text {bulk }}^{\prime} \rightarrow \mathcal{H}_{\text {bulk }}$ which-again by uniqueness-have to compose to the identity.

The bulk-boundary OPE $\beta_{\mathrm{bb}}(-, y)$ is necessarily injective. To see this, note that if $f: M \rightarrow \mathcal{H}_{\text {bulk }}$ is any $\mathcal{W} \otimes_{\mathbb{C}} \overline{\mathcal{W}}$-intertwiner such that $\beta_{\mathrm{bb}} \circ f=0$, then $f$ is an arrow from $(M, 0)$ to $\left(\mathcal{H}_{\text {bulk }}, \beta\right)$. As 0 is also an arrow between these pairs, by uniqueness we have $f=0$. This shows that the present formulation is equivalent to the one given in [53, section 3.1].

For the actual computations, the simplest case (and in many situations the only tractable case) is to take as the boundary condition the 'identity' boundary condition, i.e. the boundary condition whose space of states just consists of the $\mathcal{W}$-algebra itself. Such a boundary condition exists for the $\mathcal{W}_{1, p}$-models $[22,53]$. In that case, the objects of the category are $(\mathcal{H}, \beta)$, where $\beta$ is a bulk-boundary map $\mathcal{H} \rightarrow \mathcal{W}$. Since the image lies in $\mathcal{W}$, the centrality condition (b) in the definition of the bulk-boundary map is trivial, and the problem simplifies considerably. This approach was successfully applied to the $\mathcal{W}_{1, p}$-models in [53].

\subsection{The proposal for the bulk space of the $\mathcal{W}_{2,3}$-model}

Next, we want to repeat a similar analysis for the case of the $\mathcal{W}_{2,3}$-model. Unfortunately, as was already mentioned earlier, the $\mathcal{W}_{2,3}$-model does not have an 'identity brane' whose space of states only consists of $\mathcal{W}_{2,3}$ itself. Thus, we cannot directly apply the same method that worked for the $\mathcal{W}_{1, p}$-models. One could obviously try to apply the general formalism to a different boundary condition, but then the centrality condition (b) plays an important role, and we have not been able to characterize the most general bulk-boundary maps in any useful way.

Instead, we shall proceed slightly differently. By assumption, any $\mathcal{W}$-symmetric boundary condition has $\mathcal{W}$ as a subalgebra in its space of boundary fields $\mathcal{H}_{\text {bnd }}$. Since $\mathcal{H}_{\text {bnd }}$ is selfconjugate, it follows that $\mathcal{W}^{*}$ can be obtained as a quotient of $\mathcal{H}_{\text {bnd }}$. It seems reasonable to assume that there is at least one boundary condition, for which the disc correlator of a bulk and a boundary field is already non-degenerate in the bulk entry if we take the boundary insertions only from $\mathcal{W}$. (This is the natural analogue of the 'identity brane case'.) The two-point functions on the boundary define a natural pairing between $\mathcal{W}$ and $\mathcal{W}^{*}$. The above supposition therefore implies that the bulk-boundary map is still injective after projecting from $\mathcal{H}_{\text {bnd }}$ to $\mathcal{W}^{*}$. Obviously, for the $\mathcal{W}_{1, p}$-models $\mathcal{W}^{*} \cong \mathcal{W}$, and the distinction between $\mathcal{W}$ and $\mathcal{W}^{*}$ is immaterial.

We thus propose that the natural analogue of the 'identity brane' situation is to consider bulk-boundary maps $\beta: \mathcal{H} \rightarrow \mathcal{W}^{*}$ whose image is $\mathcal{W}^{*}$, rather than $\mathcal{W}$, and that the terminal object within this category is our desired bulk space $\left(\mathcal{H}_{\text {bulk }}, \beta_{\mathrm{bb}}\right)$. As we shall explain in section 2.3 , it satisfies a number of fairly non-trivial consistency conditions.

2.2.1. Characterizing the terminal object. As demonstrated above, let us consider the category of pairs $(\mathcal{H}, \beta)$, where $\mathcal{H}$ is a $\mathcal{W} \otimes_{\mathbb{C}} \overline{\mathcal{W}}$-representation, and $\beta: \mathcal{H} \rightarrow \mathcal{W}^{*}$ is compatible with the $\mathcal{W}$-symmetry, that is, it satisfies condition (a) of a bulk-boundary map as 
stated in section 2.1.2. The arrows are intertwiners $f: \mathcal{H} \rightarrow \mathcal{H}^{\prime}$ which make the diagram

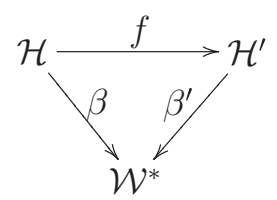

commute. We want to find a terminal object $\left(\mathcal{H}_{\text {bulk }}, \beta_{\mathrm{bb}}\right)$ in this category. As in section 2.1.2, the terminal object need not exist, but if it does it is unique and $\beta_{\mathrm{bb}}$ is injective.

It was argued in [41, section 5.1] that there is an isomorphism $\operatorname{Hom}\left(U, V^{*}\right) \rightarrow$ $\operatorname{Hom}\left(U \otimes_{f} V, \mathcal{W}^{*}\right)$. The image of the natural isomorphism $U \rightarrow U^{* *}$ under this map provides a non-degenerate pairing

$$
e v_{U}: U \otimes_{f} U^{*} \longrightarrow \mathcal{W}^{*}
$$

for all representations $U$. Let $V_{e v_{U}}(-, x): U \times U^{*} \rightarrow \mathcal{W}^{*}$ be the corresponding intertwining operator. Non-degeneracy means that for all $u \in U$ there is a $u^{\prime} \in U^{*}$ such that $V_{e v_{U}}(u, x) u^{\prime} \neq 0$, and vice versa.

Suppose that $\mathcal{W}$ has a finite number of irreducible representations and that each of these has a projective cover $\mathcal{P}(a)$. Let us denote by $\hat{\mathcal{H}}$ the space $\hat{\mathcal{H}}=\bigoplus_{a} \mathcal{P}(a) \otimes_{\mathbb{C}} \overline{\mathcal{P}}(a)^{*}$, where the direct sum is over all irreducible representations, and define the map $\hat{\beta}=\bigoplus_{a} V_{e v_{\mathcal{P}(a)}}$ : $\hat{\mathcal{H}} \rightarrow \mathcal{W}^{*}$. Let $\mathcal{H}_{\text {bulk }}=\hat{\mathcal{H}} / \operatorname{ker}(\hat{\beta})$ and let $\beta_{\text {bb }}: \mathcal{H}_{\text {bulk }} \rightarrow \mathcal{W}^{*}$ be the map induced by $\hat{\beta}$ on the quotient. We claim that

$\left(\mathcal{H}_{\text {bulk }}, \beta_{\text {bb }}\right)$ is a terminal object in the above category.

The proof proceeds analogously to the one in [53, section 3.5]. Every representation $\mathcal{H}^{\prime}$ can be written as

$$
\mathcal{H}^{\prime}=\hat{\mathcal{H}}^{\prime} / Q^{\prime} ; \quad \hat{\mathcal{H}}^{\prime}=\bigoplus_{a, b} N_{a b} \mathcal{P}(a) \otimes_{\mathbb{C}} \overline{\mathcal{P}}(b),
$$

where $N_{a b}$ are multiplicities and $Q^{\prime}$ is a sub-representation of the direct sum. Suppose that $\left(\mathcal{H}^{\prime}, \beta^{\prime}\right)$ is a pair in our category, where we write $\mathcal{H}^{\prime}$ as in (2.10). Denote the corresponding projection by $\pi^{\prime}: \hat{\mathcal{H}}^{\prime} \rightarrow \mathcal{H}^{\prime}$. If we define $\hat{\beta}^{\prime}=\beta^{\prime} \circ \pi^{\prime}$, then $\pi^{\prime}$ describes an arrow from $\left(\hat{\mathcal{H}}^{\prime}, \hat{\beta}^{\prime}\right)$ to $\left(\mathcal{H}^{\prime}, \beta^{\prime}\right)$.

Our first aim is to construct an arrow $\left(\hat{\mathcal{H}}^{\prime}, \hat{\beta}^{\prime}\right) \rightarrow\left(\mathcal{H}_{\text {bulk }}, \beta_{\mathrm{bb}}\right)$. To this end, we denote the restriction of $\hat{\beta}^{\prime}$ to the $k^{\prime}$ th summand $\mathcal{P}(a) \otimes_{\mathbb{C}} \mathcal{P}(b) \hookrightarrow \hat{\mathcal{H}}^{\prime}$ by $\hat{\beta}_{k}^{\prime}$. By the definition of $e v_{\mathcal{P}(a)}$, there exist intertwiners $f_{a, b, k}: \mathcal{P}(b) \rightarrow \mathcal{P}(a)^{*}$ such that

$$
\hat{\beta}_{k}^{\prime}=V_{e v_{\mathcal{P}(a)}} \circ\left(\operatorname{id}_{\mathcal{P}(a)} \otimes_{\mathbb{C}} f_{a, b, k}\right) .
$$

Then, $f=\bigoplus_{a} \sum_{b, k} \operatorname{id}_{\mathcal{P}(a)} \otimes_{\mathbb{C}} f_{a, b, k}: \hat{\mathcal{H}}^{\prime} \rightarrow \hat{\mathcal{H}}$ provides an arrow $f:\left(\hat{\mathcal{H}}^{\prime}, \hat{\beta}^{\prime}\right) \rightarrow(\hat{\mathcal{H}}, \hat{\beta})$. We compose this arrow with the projection $(\hat{\mathcal{H}}, \hat{\beta}) \rightarrow\left(\mathcal{H}_{\text {bulk }}, \beta_{\text {bb }}\right)$ to give the desired arrow $f^{\prime}:\left(\hat{\mathcal{H}}^{\prime}, \hat{\beta}^{\prime}\right) \rightarrow\left(\mathcal{H}_{\text {bulk }}, \beta_{\text {bb }}\right)$.

Next, we want to show that this gives rise to an arrow on $\left(\mathcal{H}^{\prime}, \beta^{\prime}\right)$. First, we note that $Q^{\prime}$ is equal to $\operatorname{ker}\left(\pi^{\prime}\right)$, and thus also $\hat{\beta}^{\prime}$ vanishes on $Q^{\prime}$. But $\hat{\beta}^{\prime}=\beta_{\text {bb }} \circ f^{\prime}$ (as $f^{\prime}: \hat{\mathcal{H}}^{\prime} \rightarrow \mathcal{H}_{\text {bulk }}$ is an arrow in our category), and since $\beta_{\mathrm{bb}}$ is injective, $f^{\prime}$ vanishes on $Q^{\prime}$. Thus, there is a well-defined map $g: \mathcal{H}^{\prime} \rightarrow \mathcal{H}_{\text {bulk }}$ on the quotient, which provides the sought-after arrow $\left(\mathcal{H}^{\prime}, \beta^{\prime}\right) \rightarrow\left(\mathcal{H}_{\text {bulk }}, \beta_{\mathrm{bb}}\right)$. Since $\beta_{\mathrm{bb}}$ is injective, this arrow is unique.

It remains to find a practical way to compute the kernel $\mathcal{N}=\operatorname{ker} \hat{\beta}$ of $\hat{\beta}: \hat{\mathcal{H}} \rightarrow \mathcal{W}^{*}$ to obtain $\mathcal{H}_{\text {bulk }}$ as a quotient. This can be done by the construction in [53, section 3.3], which we briefly review. There we described $\mathcal{N}$ as the image of all possible maps of 
projectives $\mathcal{P}(i) \otimes_{\mathbb{C}} \overline{\mathcal{P}}(j)$, for all $i, j$, into $\mathcal{N}$. For fixed $i, j$ such a map takes the form $h: \mathcal{P}(i) \otimes_{\mathbb{C}} \overline{\mathcal{P}}(j) \rightarrow \hat{\mathcal{H}}, h=\bigoplus_{a} f_{a} \otimes_{\mathbb{C}} \bar{g}_{a}^{*}$, where $f_{a}: \mathcal{P}(i) \rightarrow \mathcal{P}(a)$ and $g_{a}: \mathcal{P}(a) \rightarrow \mathcal{P}(j)^{*}$. Since

$$
\hat{\beta} \circ h=\bigoplus_{a} V_{e v_{\mathcal{P}(a)}} \circ\left(f_{a} \otimes_{\mathbb{C}} \bar{g}_{a}^{*}\right)=\sum_{a} V_{e v_{\mathcal{P}(i)}} \circ\left(\operatorname{id}_{\mathcal{P}(i)} \otimes_{\mathbb{C}}\left(\overline{g_{a} \circ f_{a}}\right)^{*}\right)
$$

$\hat{\beta} \circ h$ is zero if and only if $\sum_{a} g_{a} \circ f_{a}=0$. Since $\mathcal{N}$ itself is a quotient of a direct sum of $\mathcal{P}(i) \otimes_{\mathbb{C}} \mathcal{P}(j)$ with some multiplicities, the above construction produces the entire kernel.

If $\mathcal{P}(j)^{*} \cong \mathcal{P}(j)$, a generic example of such maps $f_{a}$ and $g_{a}$ is found as follows. Let $e: \mathcal{P}(i) \rightarrow \mathcal{P}(j)$ be any morphism. Then

$$
\left(\mathcal{P}(i) \stackrel{e}{\longrightarrow} \mathcal{P}(j) \stackrel{\text { id }}{\longrightarrow} \mathcal{P}(j) \cong \mathcal{P}(j)^{*}\right)+\left(\mathcal{P}(i) \stackrel{\text { id }}{\longrightarrow} \mathcal{P}(i) \stackrel{e}{\longrightarrow} \mathcal{P}(j) \cong \mathcal{P}(j)^{*}\right)=0 .
$$

Thus, the image of $\mathcal{P}(i) \otimes_{\mathbb{C}} \mathcal{P}(j)^{*}$ under $e \otimes_{\mathbb{C}}$ id - id $\otimes_{\mathbb{C}} \bar{e}^{*}$ lies in $\mathcal{N}$.

2.2.2. Projective covers. We now restrict our attention again to the case $\mathcal{W}=\mathcal{W}_{2,3}$. As we have just seen, the construction of $\mathcal{H}_{\text {bulk }}$ relies on the existence of projective covers for all irreducible representations. For $\mathcal{W}(h)$ with $h$ taking all values in (1.4) except $h=0$, these were given in [41] to be (1.6). The argument in [41] was based on the assumption that the projective cover of the irreducible representation $\mathcal{W}\left(\frac{-1}{24}\right)$ is $\mathcal{W}\left(\frac{-1}{24}\right)$ itself. Hence, for every representation $U$ that has a dual, also $\mathcal{W}\left(\frac{-1}{24}\right) \otimes_{f} U$ is projective (see appendix B.1 for details). By the fusion rules in [41, appendix A.4], this implies that the indecomposable representations listed in (1.6) are necessarily projective. From their composition series as given in appendix A.1, one sees that they provide the projective covers of all $\mathcal{W}(h)$ except for $h=0$.

Regarding $\mathcal{P}(0)$, one first notes that the only representations $R$ in (1.4) and (1.5) which allow for a surjection $R \rightarrow \mathcal{W}(0)$ are $\mathcal{W}(0), \mathcal{W}$ and $\mathcal{Q}$. It is verified in [41, appendix A.3] that these are not projective. On the other hand, in [55, theorem 3.24] it is stated that under certain conditions on $\mathcal{W}$, including $C_{1}$-co-finiteness, every irreducible $\mathcal{W}$-representation has a projective cover. We do not know if $\mathcal{W}_{2,3}$ satisfies these conditions, but it seems plausible to us that it does (see also [44]). We will hence assume that $\mathcal{W}(0)$ does have a projective cover. Using these assumptions as well as the result of the recent calculation of Zhu's algebra [46], it follows that $\mathcal{P}(0)$ has the composition series

$\mathcal{W}(0) \rightarrow \mathcal{W}(1) \oplus \mathcal{W}(2) \rightarrow \mathcal{W}(0) \oplus 2 \mathcal{W}(5) \oplus 2 \mathcal{W}(7) \rightarrow \mathcal{W}(1) \oplus \mathcal{W}(2) \rightarrow \mathcal{W}(0)$

The derivation is given in appendix $\mathrm{B}$, and the corresponding embedding diagram can be found in appendix A.1.

2.2.3. Computing the kernel. Next, we want to work out $\left(\mathcal{H}_{\text {bulk }}, \beta_{\mathrm{bb}}\right)$ as defined in the previous subsection explicitly. Recall from section 2.2.1 that $\hat{\mathcal{H}}$ is defined as $\hat{\mathcal{H}}=\bigoplus_{a} \mathcal{P}(a) \otimes_{\mathbb{C}} \overline{\mathcal{P}}(a)^{*}$. We want to determine the quotient space $\mathcal{H}_{\text {bulk }}=\hat{\mathcal{H}} / \operatorname{ker}(\hat{\beta})$, where

$$
\hat{\beta}=\bigoplus_{a} V_{\operatorname{ev}_{\mathcal{P}(a)}}: \hat{\mathcal{H}} \rightarrow \mathcal{W}^{*}
$$

First, we will show that one may choose representatives of vectors in $\mathcal{H}_{\text {bulk }}$ in

$$
\mathcal{H}_{\text {bulk }}^{(0)}=\bigoplus_{a} \mathcal{W}(a) \otimes_{\mathbb{C}} \overline{\mathcal{P}}(a)^{*},
$$

where $\mathcal{W}(a)$ is the top factor in the composition series of $\mathcal{P}(a)$. (This is very similar to what happened in [28] and [53].) Consider some element $\mathcal{W}(i) \otimes_{\mathbb{C}} \overline{\mathcal{W}}(j) \subset \mathcal{P}(a) \otimes_{\mathbb{C}} \overline{\mathcal{P}}(a)^{*}$ in the composition series of $\hat{\mathcal{H}}$. Since $\mathcal{P}(i)$ is the projective cover of $\mathcal{W}(i)$, it follows that there 
exists an intertwiner $e: \mathcal{P}(i) \rightarrow \mathcal{P}(a)$ such that the top factor $\mathcal{W}(i)$ in the composition series of $\mathcal{P}(i)$ is mapped to the given $\mathcal{W}(i) \subset \mathcal{P}(a)$. By (2.13) it then follows that the image of

$$
e \otimes_{\mathbb{C}} \operatorname{id}_{\overline{\mathcal{P}}(a)}-\operatorname{id}_{\mathcal{P}(i)} \otimes_{\mathbb{C}} \bar{e}^{*}
$$

lies in the kernel of $\hat{\beta}$. Thus in $\mathcal{H}_{\text {bulk }}$, any vector in $\mathcal{W}(i) \otimes_{\mathbb{C}} \overline{\mathcal{W}}(j) \subset \mathcal{P}(a) \otimes_{\mathbb{C}} \overline{\mathcal{P}}(a)^{*}$, where $\mathcal{W}(i)$ is not the top component of $\mathcal{P}(a)$, lies in the same equivalence class as a vector in $\mathcal{W}(i) \otimes_{\mathbb{C}} \bar{e}^{*}(\overline{\mathcal{W}}(j)) \subset \mathcal{P}(i) \otimes_{\mathbb{C}} \overline{\mathcal{P}}(i)^{*}$, where $\mathcal{W}(i)$ is the top component of $\mathcal{P}(i)$. Thus, we have shown that $\mathcal{H}_{\text {bulk }}$ is at most as big as $\mathcal{H}_{\text {bulk }}^{(0)}$.

We can collect the terms of $\hat{\mathcal{H}}$ into sectors

$$
\hat{\mathcal{H}}=\hat{\mathcal{H}}_{0} \oplus \hat{\mathcal{H}}_{\frac{1}{8}} \oplus \hat{\mathcal{H}}_{\frac{5}{8}} \oplus \hat{\mathcal{H}}_{\frac{1}{3}} \oplus \hat{\mathcal{H}}_{\frac{35}{24}} \oplus \hat{\mathcal{H}}_{\frac{-1}{24}}
$$

with

$$
\begin{array}{ll}
\hat{\mathcal{H}}_{\frac{-1}{24}}=\mathcal{W}\left(\frac{-1}{24}\right) \otimes_{\mathbb{C}} \overline{\mathcal{W}}\left(\frac{-1}{24}\right)^{*} & \hat{\mathcal{H}}_{\frac{35}{24}}=\mathcal{W}\left(\frac{35}{24}\right) \otimes_{\mathbb{C}} \overline{\mathcal{W}}\left(\frac{35}{24}\right)^{*} \\
\hat{\mathcal{H}}_{\frac{1}{3}}=\bigoplus_{a=\frac{1}{3}, \frac{10}{3}} \mathcal{P}(a) \otimes_{\mathbb{C}} \overline{\mathcal{P}}(a)^{*} & \hat{\mathcal{H}}_{\frac{5}{8}}=\bigoplus_{a=\frac{5}{8}, \frac{21}{8}} \mathcal{P}(a) \otimes_{\mathbb{C}} \overline{\mathcal{P}}(a)^{*} \\
\hat{\mathcal{H}}_{\frac{1}{8}}=\bigoplus_{a=\frac{1}{8}, \frac{33}{8}} \mathcal{P}(a) \otimes_{\mathbb{C}} \overline{\mathcal{P}}(a)^{*} & \hat{\mathcal{H}}_{0}=\bigoplus_{a=0,1,2,5,7} \mathcal{P}(a) \otimes_{\mathbb{C}} \overline{\mathcal{P}}(a)^{*}
\end{array}
$$

It is easy to see that the kernel of $\hat{\beta}$ does not mix the different sectors in (2.18), and we may therefore consider each of them in turn. It follows from the arguments of [53, section 4.3 and appendix D] that in all sectors, except possibly for $\hat{\mathcal{H}}_{0}$, all relations of the quotient space $\mathcal{H}_{\text {bulk }}=\hat{\mathcal{H}} / \operatorname{ker}(\hat{\beta})$ are taken into account by (2.17) and that $\mathcal{H}_{\text {bulk }}$ is isomorphic (as an $\left(L_{0}^{\text {diag }}, \bar{L}_{0}^{\text {diag }}\right)$-graded vector space, see $\left.(1.1)\right)$ to $\mathcal{H}_{\text {bulk }}^{(0)}$. We believe that this will also be the case for $\hat{\mathcal{H}}_{0}$, but we have no proof. We therefore conjecture that $\mathcal{H}_{\text {bulk }}^{(0)}$ is not just an upper bound for $\mathcal{H}_{\text {bulk }}$, but actually isomorphic to it. This gives then the explicit description of $\mathcal{H}_{\text {bulk }}$ that was mentioned before. (The bulk-boundary map $\beta_{\mathrm{bb}}$ is the one induced from $\hat{\beta}$ under the quotient map $\hat{\mathcal{H}} \rightarrow \mathcal{H}_{\text {bulk. }}$.

\subsection{Properties of the resulting bulk space}

Next, we want to describe the resulting bulk space $\mathcal{H}_{\text {bulk }}$ in some more detail. First, we want to explain how $\mathcal{W} \otimes_{\mathbb{C}} \overline{\mathcal{W}}$ acts on it. Given that we have a description of $\mathcal{H}_{\text {bulk }}$ as a quotient by $\mathcal{N}$ of $\hat{\mathcal{H}}$, this can now be easily deduced. Since $\hat{\mathcal{H}}$ has a decomposition as in (2.18), we get a similar decomposition for $\mathcal{H}_{\text {bulk }}$, which we write as

$$
\mathcal{H}_{\text {bulk }}=\hat{\mathcal{H}} / \mathcal{N}=\mathcal{H}_{0} \oplus \mathcal{H}_{\frac{1}{8}} \oplus \mathcal{H}_{\frac{5}{8}} \oplus \mathcal{H}_{\frac{1}{3}} \oplus \mathcal{H}_{\frac{-1}{24}} \oplus \mathcal{H}_{\frac{35}{24}}
$$

In order to describe the resulting structure we analyse, sector by sector, their composition series, following the method outlined in section 1.3. This is to say, we identify first the largest direct sum of irreducible sub-representations; then we quotient by these and find the largest direct sum of irreducible sub-representations in the quotient, etc. The situation is obviously simplest for $\mathcal{H}_{-1 / 24}$ and $\mathcal{H}_{35 / 24}$ since they are already, by themselves, irreducible. As a consequence, the quotient is trivial for these sectors, and the resulting composition series just consists of one term.

2.3.1. The edge of the Kac table. The situation is more interesting for those sectors that come from the 'edge of the Kac table', i.e. the sectors $\mathcal{H}_{1 / 8}, \mathcal{H}_{1 / 3}$ and $\mathcal{H}_{5 / 8}$. In the following, we shall only show the calculation for the first case $\left(h=\frac{1}{8}\right)$ - the other cases then follow upon replacing $\left\{\frac{1}{8}, \frac{33}{8}\right\}$ with $\left\{\frac{5}{8}, \frac{21}{8}\right\}$ or $\left\{\frac{1}{3}, \frac{10}{3}\right\}$, respectively. 
As we have explained above, the representatives are described by $\mathcal{H}_{1 / 8}^{(0)}$, see equation (2.16). It is then not difficult to show that the maximal fully reducible subrepresentation of $M_{1}\left(\frac{1}{8}\right)=\mathcal{H}_{1 / 8}$ is given by

$$
R_{1}\left(\frac{1}{8}\right)=\bigoplus_{i=\frac{1}{8}, \frac{33}{8}} \mathcal{W}(i) \otimes_{\mathbb{C}} \overline{\mathcal{W}}(i)
$$

where $\overline{\mathcal{W}}(i)$ comes from the bottom entry in $\overline{\mathcal{P}}(i)$.

Next, we consider the quotient $M_{2}\left(\frac{1}{8}\right)=M_{1}\left(\frac{1}{8}\right) / R_{1}\left(\frac{1}{8}\right)$ and repeat the analysis. The maximal fully reducible sub-representation of $M_{2}\left(\frac{1}{8}\right)$ is

$$
R_{2}\left(\frac{1}{8}\right)=2 \mathcal{W}\left(\frac{1}{8}\right) \otimes_{\mathbb{C}} \overline{\mathcal{W}}\left(\frac{33}{8}\right) \oplus 2 \mathcal{W}\left(\frac{33}{8}\right) \otimes_{\mathbb{C}} \overline{\mathcal{W}}\left(\frac{1}{8}\right)
$$

These arise from the middle lines in the embedding diagrams of the corresponding projective covers $\overline{\mathcal{P}}(i)$. Finally, the maximal fully reducible sub-representation of $M_{3}\left(\frac{1}{8}\right)=$ $M_{2}\left(\frac{1}{8}\right) / R_{2}\left(\frac{1}{8}\right)$ equals

$$
R_{3}\left(\frac{1}{8}\right)=\bigoplus_{i=\frac{1}{8}, \frac{33}{8}} \mathcal{W}(i) \otimes_{\mathbb{C}} \overline{\mathcal{W}}(i),
$$

which comes from the top entries of $\overline{\mathcal{P}}(i)$. Thus, we obtain precisely the composition series given in (1.18).

2.3.2. The interior of the Kac table. This leaves us with the sector $\mathcal{H}_{0}$, for which the analysis is more complicated. Proceeding as before we find that the maximal fully reducible sub-representation of $M_{1}(0)=\mathcal{H}_{0}$ is given by

$$
R_{1}(0)=\bigoplus_{i=0,1,2,5,7} \mathcal{W}(i) \otimes_{\mathbb{C}} \overline{\mathcal{W}}(i)
$$

As before, these states arise from the bottom component in $\overline{\mathcal{P}}(i)^{*}$.

Next, we find that the maximal fully reducible sub-representation of $M_{2}(0)=$ $M_{1}(0) / R_{1}(0)$ is

$$
\begin{aligned}
R_{2}(0)=(\mathcal{W}(1) & \oplus \mathcal{W}(2)) \otimes_{\mathbb{C}}(2 \overline{\mathcal{W}}(5) \oplus 2 \overline{\mathcal{W}}(7) \oplus \overline{\mathcal{W}}(0)) \\
& \oplus(\mathcal{W}(5) \oplus \mathcal{W}(7)) \otimes_{\mathbb{C}}(2 \overline{\mathcal{W}}(2) \oplus 2 \overline{\mathcal{W}}(1)) \\
& \oplus \mathcal{W}(0) \otimes_{\mathbb{C}}(\overline{\mathcal{W}}(1) \oplus \overline{\mathcal{W}}(2))
\end{aligned}
$$

while that of $M_{3}(0)=M_{2}(0) / R_{2}(0)$ is

$$
\begin{aligned}
R_{3}(0)=\mathcal{W}(1) & \otimes_{\mathbb{C}}(2 \overline{\mathcal{W}}(1) \oplus 4 \overline{\mathcal{W}}(2)) \\
& \oplus \mathcal{W}(2) \otimes_{\mathbb{C}}(2 \overline{\mathcal{W}}(2) \oplus 4 \overline{\mathcal{W}}(1)) \\
& \oplus \mathcal{W}(0) \otimes_{\mathbb{C}}(\overline{\mathcal{W}}(0) \oplus 2 \overline{\mathcal{W}}(5) \oplus 2 \overline{\mathcal{W}}(7)) \\
& \oplus \mathcal{W}(5) \otimes_{\mathbb{C}}(2 \overline{\mathcal{W}}(0) \oplus 2 \overline{\mathcal{W}}(5) \oplus 4 \overline{\mathcal{W}}(7)) \\
& \oplus \mathcal{W}(7) \otimes_{\mathbb{C}}(2 \overline{\mathcal{W}}(0) \oplus 2 \overline{\mathcal{W}}(7) \oplus 4 \overline{\mathcal{W}}(5)) .
\end{aligned}
$$

Similarly, the maximal fully reducible sub-representation of $M_{4}(0)=M_{3}(0) / R_{3}(0)$ is $R_{4}(0)=R_{2}(0)$, while that of $M_{5}(0)=M_{4}(0) / R_{4}(0)$ equals $R_{5}(0)=R_{1}(0)$. This agrees precisely with what we claimed in (1.19). 
2.3.3. Consistency conditions. Finally, let us comment on the consistency conditions our answer satisfies. None of the properties discussed below is built into our ansatz from the start, and they give strong support to our proposed bulk space.

Modular invariance. Given the composition series (1.16), (1.18) and (1.19), it is straightforward to work out the partition function of $\mathcal{H}_{\text {bulk }}$, resulting in

$$
\begin{aligned}
Z(q) & =\sum_{i} \chi_{\mathcal{W}(i)}(q) \cdot \chi_{\mathcal{P}(i)}(\bar{q}) \\
& =(q \bar{q})^{-1 / 24}+3+2(q \bar{q})^{1 / 8}+2(q \bar{q})^{1 / 3}+(q+\bar{q}) \cdot(q \bar{q})^{-1 / 24}+2(q+\bar{q})+\cdots,
\end{aligned}
$$

where the sum runs over all 13 irreducibles (including $\mathcal{W}(0)$ ). This partition function was already proposed in [41, section 4], at least up to the multiplicity of $(q \bar{q})^{0}$, which was left as an arbitrary positive integer there. As was mentioned in [41], $Z(q)$ is modular invariant (see also [35]).

Self-conjugacy. The space of bulk states $\mathcal{H}_{\text {bulk }}$ is actually isomorphic to its conjugate representation, $\mathcal{H}_{\mathrm{bulk}}^{*}$. This is necessary in order for the bulk theory to have a non-degenerate (bulk) two-point function.

Stress tensor. Curiously, the composition series (1.19) of $\mathcal{H}_{\text {bulk }}$ shows that $\mathcal{H}_{\text {bulk }}$ does not contain $\mathcal{W} \otimes_{\mathbb{C}} \overline{\mathcal{W}}$ as a sub-representation. To see this, we note that the composition series of $\mathcal{W} \otimes_{\mathbb{C}} \overline{\mathcal{W}}$ is

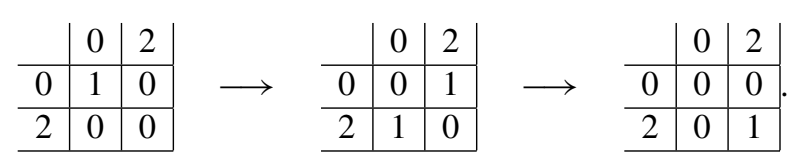

In order to embed $\mathcal{W} \otimes_{\mathbb{C}} \overline{\mathcal{W}}$ into $\mathcal{H}_{\text {bulk }}$, we need to map the level 0 subspace $\mathcal{W}(0) \otimes_{\mathbb{C}} \overline{\mathcal{W}}(0)$ to the summand $\mathcal{W}(0) \otimes_{\mathbb{C}} \overline{\mathcal{W}}(0)$ of $\mathcal{H}_{\text {bulk }}$ at level 2. However, the composition series (A.1) of $\mathcal{P}(0)$ shows that from there one can reach e.g. $\mathcal{W}(0) \otimes_{\mathbb{C}} \overline{\mathcal{W}}(1)$, which is not contained in $\mathcal{W} \otimes_{\mathbb{C}} \overline{\mathcal{W}}$. Nonetheless, $\mathcal{H}_{\text {bulk }}$ does still contain a holomorphic field of weight $(2,0)$ and an anti-holomorphic field of weight $(0,2)$, which form the candidate stress tensor. For example, a representative of the equivalence class of the state of weight $(2,0)$ at level 3 of $\mathcal{H}_{\text {bulk }}$ (see $(1.19))$ is $\mathcal{W}(2) \otimes_{\mathbb{C}} \overline{\mathcal{W}}(0) \subset \mathcal{P}(2) \otimes_{\mathbb{C}} \overline{\mathcal{P}}(2)^{*}$, where $\overline{\mathcal{W}}(0)$ sits at level 3. Acting with $\bar{L}_{-1}$ gives a state of weight $(2,1)$, where the factor with weight 1 has to be at level 4 of $\mathcal{P}(2)$, which, however, does not contain such a state. Thus, the state of weight $(2,0)$ really defines a holomorphic field.

As an aside, we note that this argument also suggests that the state of weight $(1,0)$ at level 3 of $\mathcal{H}_{\text {bulk }}$ is not holomorphic, as acting with $\bar{L}_{-1}$ on its representative $\mathcal{W}(1) \otimes_{\mathbb{C}} \overline{\mathcal{W}}(0) \subset \mathcal{P}(1) \otimes_{\mathbb{C}} \overline{\mathcal{P}}(1)^{*}$, where $\overline{\mathcal{W}}(0)$ sits at level 3 , gives a state of weight $(1,1)$ in $\mathcal{W}(1) \otimes_{\mathbb{C}} \overline{\mathcal{W}}(1) \subset \mathcal{P}(1) \otimes_{\mathbb{C}} \overline{\mathcal{P}}(1)^{*}$, where $\overline{\mathcal{W}}(1)$ sits at level 4, and it seems highly plausible that this state is non-zero.

Boundary states. As a final consistency check, we can study the boundary states one can construct within $\mathcal{H}_{\text {bulk. }}$. In particular, as we shall show in the following section, we are able to reproduce the boundary theory of [41] in this manner.

\section{The boundary state analysis}

In this section, we want to study how the boundary conditions that were proposed in [41] fit into the present analysis. In particular, we want to show how the corresponding boundary states can be constructed in our proposed bulk theory $\mathcal{H}_{\text {bulk }}$. 


\subsection{Reconstructing the boundary states}

Let us begin by identifying the Ishibashi states and their overlaps that reproduce the annulus partition functions of [41]; this first part of the analysis does not require any detailed knowledge about the bulk space $\mathcal{H}_{\text {bulk }}$. The basic idea of the analysis is simple. Given the proposal of [41], we know the annulus partition functions in the 'open string sector'. More specifically, if $\mathcal{R}$ and $\mathcal{R}^{\prime}$ label two consistent boundary conditions, then the open string annulus amplitude is simply

$$
\chi_{\mathcal{R} \otimes_{f} \mathcal{R}^{\prime *}}(\tilde{q}),
$$

where $\chi_{\mathcal{S}}$ denotes the character of $\mathcal{S}$, and $\tilde{q}=\exp (-2 \pi \mathrm{i} / \tau)$ is the open string modular parameter. Thus, the overlap of the corresponding boundary states $\| \mathcal{R}\rangle\rangle$ and $\left.\left.\| \mathcal{R}^{\prime}\right\rangle\right\rangle$ must equal

$$
\left\langle\left\langle\mathcal{R}\left\|q^{L_{0}+\bar{L}_{0}}\right\| \mathcal{R}^{\prime}\right\rangle\right\rangle=\chi_{\mathcal{R} \otimes_{f} \mathcal{R}^{*}}(\tilde{q}),
$$

i.e. the modular $S$-transform of (3.1) — see appendix A.2 for explicit formulae, or [35, 38] for the formula for general $\mathcal{W}_{p, q}$-models.

By considering the different powers of $q$ that appear in (3.2), it is clear which contribution arises from which sector of $\mathcal{H}_{\text {bulk }}$ in (2.20). We can thus identify the overlaps of the various different Ishibashi states that appear. As was explained in section 1.4, the annulus amplitudes can be described in terms of ten Ishibashi states, reflecting the fact that the overlaps have a two-dimensional null ideal, see equation (1.9). We should stress, however, that this does not mean that the actual boundary states can be written in terms of these ten Ishibashi states. It only means that for the calculation of the annulus amplitudes, only the coupling to these ten Ishibashi states is required. (In fact, it seems natural to assume that the actual boundary states will depend on two more Ishibashi states, but we have not managed to prove this.)

So far, we have not used any detailed information about the structure of the bulk spectrum. The non-trivial consistency condition now arises from the requirement that the Ishibashi states (1.20) and their overlaps (1.21) can indeed be obtained within $\mathcal{H}_{\text {bulk }}$. In order to see that this is possible, we begin by classifying the most general Ishibashi states in $\mathcal{H}_{\text {bulk }}$.

\subsection{The Ishibashi states}

As was explained in [53, section 5.1], we may think of the Ishibashi states in terms of 'Ishibashi morphisms'. Indeed, each Ishibashi state defines (and is defined by) a 'bulk-boundary pairing' $\mathcal{H}_{\text {bulk }} \times \mathcal{W} \rightarrow \mathbb{C}$ that is compatible with the $\mathcal{W}$ action. Since the bulk space $\mathcal{H}_{\text {bulk }}$ is the quotient of $\hat{\mathcal{H}}$ by $\mathcal{N}$, the space of all Ishibashi intertwiners consists of those bulk-boundary pairings on $\hat{\mathcal{H}}$ that vanish on $\mathcal{N}$. It was furthermore shown in [53] that every such bulk boundary pairing can be written as

$$
b_{\rho}\left(u \otimes_{\mathbb{C}} \bar{v}, w\right)=\sum_{a}\left(\operatorname{ev}_{\mathcal{P}(a)}\left(\rho_{a}\left(u_{a}\right), \bar{v}_{a}\right)\right)(w)
$$

where $\rho$ is an intertwiner $\rho=\bigoplus_{a} \rho_{a}: \mathcal{P}(a) \rightarrow \mathcal{P}(a)$. Here, $u \otimes_{\mathbb{C}} \bar{v}=\bigoplus_{a} u_{a} \otimes_{\mathbb{C}} \bar{v}_{a} \in \hat{\mathcal{H}}$, and $w \in \mathcal{W}$. Furthermore, as also explained in [53], the condition to vanish on $\mathcal{N}$ can be analysed by very similar methods as above in section 2.2.1. The bulk boundary map $b_{\rho}$ vanishes on $\mathcal{N}$ if and only if

$$
\sum_{a} g_{a} \circ \rho_{a} \circ f_{a}=0
$$

for all $f_{a}: \mathcal{P}(i) \rightarrow \mathcal{P}(a)$ and $g_{a}: \mathcal{P}(a) \rightarrow \mathcal{P}(j)$ such that $\left(\bigoplus_{a} f_{a} \otimes_{\mathbb{C}} \bar{g}_{a}^{*}\right)\left(\mathcal{P}(i) \otimes_{\mathbb{C}} \overline{\mathcal{P}}(j)^{*}\right)$ lies in $\mathcal{N}$. 
As is familiar from the usual rational case, we can analyse the Ishibashi states sector by sector. Again, the situation is easiest for the sectors $\mathcal{H}_{-1 / 24}$ and $\mathcal{H}_{35 / 24}$, corresponding to the irreducible representations at the corner of the Kac table. In either case, Schur's lemma implies that there is only a one-dimensional space of intertwiners, and since $\mathcal{N}$ only intersects these sectors in 0 , there is no additional constraint to worry about. Thus, we have one Ishibashi state from each of these two sectors.

3.2.1. The edge of the Kac table. The situation is more interesting for the sectors from the 'edge of the Kac table', i.e. the sectors $\mathcal{H}_{1 / 8}, \mathcal{H}_{5 / 8}$ and $\mathcal{H}_{1 / 3}$. For concreteness, let us again only consider the case of $\mathcal{H}_{1 / 8}$; the situation in the other sectors is completely analogous. First, we recall that

$$
\hat{\mathcal{H}}_{\frac{1}{8}}=\left(\mathcal{P}\left(\frac{1}{8}\right) \otimes_{\mathbb{C}} \overline{\mathcal{P}}\left(\frac{1}{8}\right)^{*}\right) \oplus\left(\mathcal{P}\left(\frac{33}{8}\right) \otimes_{\mathbb{C}} \overline{\mathcal{P}}\left(\frac{33}{8}\right)^{*}\right) .
$$

As was explained in [41], the space of intertwiners $\rho: \mathcal{P}\left(\frac{1}{8}\right) \rightarrow \mathcal{P}\left(\frac{1}{8}\right)$ is two dimensional, and similarly for $\mathcal{P}\left(\frac{33}{8}\right)$. Thus, in addition to the identity intertwiner $\mathrm{id}_{a}$, there is one linearly independent intertwiner which we denote by $n_{a} \equiv e_{2}^{a \rightarrow a}$; our notation for the intertwiners is explained in appendix A.1. The intertwiner $\operatorname{id}_{a}$ acts as the identity on $\mathcal{P}(a)$, and as zero on the other summand in (3.5), and similarly for $n_{a}$. In total, we therefore have four such intertwiners, and we write the most general ansatz as

$$
\rho=\rho_{\frac{1}{8}} \oplus \rho_{\frac{33}{8}}=\bigoplus_{a=\frac{1}{8}, \frac{33}{8}} A_{a}^{(1)} \mathrm{id}^{a}+A_{a}^{(2)} n^{a} .
$$

The space $\mathcal{N}$ by which we have to quotient out $\hat{\mathcal{H}}_{1 / 8}$ is generated by

$$
\left(e_{1 ; \alpha}^{a \rightarrow b} \otimes_{\mathbb{C}} \mathrm{id}-\mathrm{id} \otimes_{\mathbb{C}}\left(e_{1 ; \alpha}^{a \rightarrow b}\right)^{*}\right)\left(\mathcal{P}(a) \otimes_{\mathbb{C}} \overline{\mathcal{P}}(b)^{*}\right)
$$

where either $\left(a=\frac{1}{8}\right.$ and $b=\frac{33}{8}$ ) or $\left(a=\frac{33}{8}\right.$ and $b=\frac{1}{8}$ ), and $\alpha$ denotes the two different choices of such intertwiners. (This is because the other relations of the form (2.13), involving intertwiners of higher degree, are a consequence of these.) By (3.4), the intertwiner $\rho$ vanishes on (3.7) if and only if for each $\alpha$ we have on $\mathcal{P}(a)$ :

$$
\rho_{b} \circ e_{1 ; \alpha}^{a \rightarrow b}-\left(e_{1 ; \alpha}^{a \rightarrow b}\right) \circ \rho_{a}=0 .
$$

Thus, there is a three-dimensional solution space with basis

$$
\rho^{(1)}=\mathrm{id}^{\frac{1}{8}}+\mathrm{id}^{\frac{33}{8}}, \quad \rho^{(a, 2)}=n^{a}, \quad a=\frac{1}{8}, \frac{33}{8},
$$

since $e_{1 ; \alpha}^{a \rightarrow b} \circ n^{a}=n^{b} \circ e_{1 ; \alpha}^{a \rightarrow b}=0$. This analysis is completely analogous to the discussion in [53].

3.2.2. The interior of the Kac table. This leaves us with analysing the Ishibashi morphisms in the sector $\mathcal{H}_{0}$, corresponding to the 'interior of the Kac table'. The following calculations make frequent reference to the embedding diagrams (A.1)-(A.3) of the projective representations $\mathcal{P}(0), \mathcal{P}(1), \mathcal{P}(2), \mathcal{P}(5)$ and $\mathcal{P}(7)$, and of our conventions regarding intertwiners described there. As is apparent from these embedding diagrams, the space of intertwiners of each of the projective representations $\mathcal{P}(a)$ with $a \in\{1,2,5,7\}$ is four dimensional. For each such $\mathcal{P}(a)$ let id ${ }^{a}$ be the identity intertwiner, while $e_{4}^{a \rightarrow a}$ denotes the unique intertwiner of degree 4. The remaining two intertwiners have degree 2 , and will be denoted by $e_{2 ; \alpha}^{a \rightarrow a}$, where $\alpha$ takes the appropriate two values out of $\{1,2,5,7\}$, as explained in appendix A.1. The most general ansatz for an intertwiner $\mathcal{P}(a) \rightarrow \mathcal{P}(a), a=1,2,5,7$ is therefore

$$
\rho_{a}=A_{a}^{\mathrm{id}} \mathrm{id}^{a}+\sum_{\alpha} A_{a}^{\alpha} e_{2 ; \alpha}^{a \rightarrow a}+A_{a}^{4} e_{4}^{a \rightarrow a} .
$$


For the case of $\mathcal{P}(0)$, the space of intertwiners from $\mathcal{P}(0)$ to itself is three dimensional; apart from the identity intertwiner $\mathrm{id}^{0}$, there are the intertwiners $e_{2}^{0 \rightarrow 0}$ and $e_{4}^{0 \rightarrow 0}$ of degrees 2 and 4 , respectively. The most general ansatz for an intertwiner $\mathcal{P}(0) \rightarrow \mathcal{P}(0)$ is therefore

$$
\rho_{0}=A_{0}^{\mathrm{id}} \mathrm{id}^{0}+A_{0}^{2} e_{2}^{0 \rightarrow 0}+A_{0}^{4} e_{4}^{0 \rightarrow 0} .
$$

Before taking into account the constraints coming from $\mathcal{N}$, the number of intertwiners we need to consider in the sector $\hat{\mathcal{H}}_{0}$ is therefore $19=4 \times 4+3$.

The intersection of $\mathcal{N}$ with $\hat{\mathcal{H}}_{0}$ is again generated by the relations of the form (2.13) involving intertwiners of degree 1 :

$$
\begin{aligned}
& \left(e_{1 ; \beta}^{a \rightarrow b} \otimes_{\mathbb{C}} \mathrm{id}^{b}-\mathrm{id}^{a} \otimes_{\mathbb{C}}\left(\overline{e_{1 ; \beta}^{a \rightarrow b}}\right)^{*}\right)\left(\mathcal{P}(a) \otimes_{\mathbb{C}} \overline{\mathcal{P}}(b)^{*}\right) \\
& \left(e_{1}^{0 \rightarrow d} \otimes_{\mathbb{C}} \mathrm{id}^{d}-\mathrm{id}^{0} \otimes_{\mathbb{C}}\left(\overline{e_{1}^{0 \rightarrow d}}\right)^{*}\right)\left(\mathcal{P}(0) \otimes_{\mathbb{C}} \overline{\mathcal{P}}(d)^{*}\right) \\
& \left(e_{1}^{d \rightarrow 0} \otimes_{\mathbb{C}} \mathrm{id}^{0}-\mathrm{id}^{d} \otimes_{\mathbb{C}}\left(\overline{e_{1}^{d \rightarrow 0}}\right)^{*}\right)\left(\mathcal{P}(d) \otimes_{\mathbb{C}} \overline{\mathcal{P}}(0)^{*}\right),
\end{aligned}
$$

where $(a, b)=(1,5),(1,7),(2,5),(2,7)$ or $(a, b)=(5,1),(7,1),(5,2),(7,2)$ and $d \in$ $\{1,2\}$. By (3.4), the intertwiner $\rho$ vanishes on (3.7) if and only if

$$
\begin{array}{ll}
\rho_{b} \circ e_{1 ; \beta}^{a \rightarrow b}-e_{1 ; \beta}^{a \rightarrow b} \circ \rho_{a}=0 & \text { on } \mathcal{P}(a), \\
\rho_{d} \circ e_{1}^{0 \rightarrow d}-e_{1}^{0 \rightarrow d} \circ \rho_{0}=0 & \text { on } \mathcal{P}(0), \\
\rho_{0} \circ e_{1}^{d \rightarrow 0}-e_{1}^{d \rightarrow 0} \circ \rho_{d}=0 & \text { on } \mathcal{P}(d) .
\end{array}
$$

Evaluating the first equation of (3.13) we get

$$
\begin{aligned}
0 & =\rho_{b} \circ e_{1 ; \beta}^{a \rightarrow b}-e_{1 ; \beta}^{a \rightarrow b} \circ \rho_{a} \\
& =\left(A_{b}^{\mathrm{id}} \mathrm{id}^{b}+\sum_{\eta} A_{b}^{\eta} e_{2 ; \eta}^{b \rightarrow b}+A_{b}^{4} e_{4}^{b \rightarrow b}\right) \circ e_{1 ; \beta}^{a \rightarrow b}-e_{1 ; \beta}^{a \rightarrow b} \circ\left(A_{a}^{\mathrm{id}} \mathrm{id}^{a}+\sum_{\eta} A_{a}^{\eta} e_{2 ; \eta}^{a \rightarrow a}+A_{a}^{4} e_{4}^{a \rightarrow a}\right) \\
& =\left(A_{b}^{\mathrm{id}}-A_{a}^{\mathrm{id}}\right) e_{1 ; \beta}^{a \rightarrow b}+\sum_{\gamma}\left(\sum_{\eta} A_{b}^{\eta} C_{\eta \beta}^{\gamma}-\sum_{\eta} A_{a}^{\eta} \hat{C}_{\beta \eta}^{\gamma}\right) e_{3 ; \gamma}^{a \rightarrow b},
\end{aligned}
$$

where we have used that $e_{4}^{b \rightarrow b} \circ e_{1 ; \beta}^{a \rightarrow b}=0=e_{1 ; \beta}^{a \rightarrow b} \circ e_{4}^{a \rightarrow a}$, as well as defined the structure constants via (see appendix A.1 for some examples):

$$
e_{2 ; \alpha}^{b \rightarrow b} \circ e_{1 ; \beta}^{a \rightarrow b}=\sum_{\gamma} C_{\alpha, \beta}^{\gamma} e_{3 ; \gamma}^{a \rightarrow b}, \quad e_{1 ; \beta}^{a \rightarrow b} \circ e_{2 ; \alpha}^{a \rightarrow a}=\sum_{\gamma} \hat{C}_{\beta, \alpha}^{\gamma} e_{3 ; \gamma}^{a \rightarrow b} .
$$

The second and third equations of (3.13) lead to equivalent relations. Concentrating on the former we obtain

$$
\begin{aligned}
0 & =\rho_{d} \circ e_{1}^{0 \rightarrow d}-e_{1}^{0 \rightarrow d} \circ \rho_{0} \\
& =\left(A_{d}^{\mathrm{id}} \mathrm{id}^{d}+A_{d}^{5} e_{2,5}^{d \rightarrow d}+A_{d}^{7} e_{2,7}^{d \rightarrow d}+A_{d}^{4} e_{4}^{d \rightarrow d}\right) \circ e_{1}^{0 \rightarrow d}-e_{1}^{0 \rightarrow d} \circ\left(A_{0}^{\mathrm{id}} \mathrm{id}^{0}+A_{0}^{2} e_{2}^{0 \rightarrow 0}+A_{0}^{4} e_{4}^{0 \rightarrow 0}\right) \\
& =\left(A_{d}^{\mathrm{id}}-A_{0}^{\mathrm{id}}\right) e_{1}^{0 \rightarrow d}+\left(A_{d}^{5}+A_{d}^{7}-A_{0}^{2}\right) e_{3}^{0 \rightarrow d},
\end{aligned}
$$

where we have again used that $e_{4}^{d \rightarrow d} \circ e_{1}^{0 \rightarrow d}=0=e_{1}^{0 \rightarrow d} \circ e_{4}^{0 \rightarrow 0}$. A basis for the space of solutions is given by the following nine Ishibashi morphisms ${ }^{8}$ :

$$
\begin{aligned}
& \rho^{(\mathrm{id})}=\mathrm{id}^{0}+\mathrm{id}^{1}+\mathrm{id}^{2}+\mathrm{id}^{5}+\mathrm{id}^{7} \\
& \rho^{(\mu)}=e_{2}^{0 \rightarrow 0}+e_{2,7}^{1 \rightarrow 1}+e_{2,5}^{2 \overrightarrow{5}^{2}}+e_{2,2}^{5 \rightarrow 5}+e_{2,1}^{7 \rightarrow 7} \\
& \rho^{(\nu)}=e_{2}^{0 \rightarrow 0}+e_{2,5}^{1 \rightarrow 1}+e_{2,7}^{2 \vec{P}^{2}}+e_{2,1}^{5 \rightarrow 5}+e_{2,2}^{7 \rightarrow 7} \\
& \rho^{(\delta)}=e_{2,5}^{2 \rightarrow^{2}}-e_{2,7}^{2 \rightarrow^{2}}+e_{2,2}^{5 \rightarrow 5}-e_{2,2}^{7 \rightarrow 7} \\
& \rho^{\left(\sigma_{i}\right)}=e_{4}^{i \rightarrow i} \quad i=0,1,2,5,7 .
\end{aligned}
$$

8 We believe that by an appropriate rescaling of the intertwiners, the coefficients $C_{\alpha, \beta}^{\gamma}$ and $\hat{C}_{\beta, \alpha}^{\gamma}$ can be chosen to be either 0 or 1 . This was used to arrive at (3.17). 


\subsection{Overlaps of Ishibashi states}

In order to identify the Ishibashi states (1.20) with linear combinations of the Ishibashi states corresponding to the intertwiners (3.17), we need to determine the different overlaps between the latter. Obviously, the overlaps between Ishibashi states from different sectors vanish, but since there is generically more than one Ishibashi state in each sector, the relative overlaps between them are more complicated. The first step in identifying the structure of these overlaps is to understand the relation between the Ishibashi morphisms $\rho$ and the corresponding Ishibashi states $|\rho\rangle\rangle$, thought of as elements in a completion of $\mathcal{H}_{\text {bulk }}$. Suppose that $w \in \mathcal{H}_{\text {bulk }}$ is an arbitrary bulk state, then we have

$$
\left.b_{\rho}(w, \Omega)=B(|\rho\rangle), w\right),
$$

where $B(-,-)$ is the bulk two-point function and $b_{\rho}$ is defined by (3.3). That $\left.|\rho\rangle\right\rangle$ is annihilated by $\left(W_{m}-(-1)^{h_{W}} \bar{W}_{-m}\right)$ is related to the fact that $b_{\rho}(-, \Omega)$ is a chiral two-point block on the sphere (see e.g. [53, appendix A]). Since the bulk two-point function is non-degenerate, it follows that $\rho$ determines uniquely the corresponding Ishibashi state $|\rho\rangle\rangle$, and vice versa. Given the knowledge of the corresponding Ishibashi state, we can then work out the cylinder overlaps since we have

$$
\left.\left.\left\langle\left\langle\rho_{1}\left|q^{L_{0}+\bar{L}_{0}}\right| \rho_{2}\right\rangle\right\rangle=B\left(\left|\rho_{1}\right\rangle\right\rangle, q^{L_{0}+\bar{L}_{0}}\left|\rho_{2}\right\rangle\right\rangle\right) .
$$

It follows from the definition of (3.3) that $b_{\rho}\left(u \otimes_{\mathbb{C}} \bar{v}, \Omega\right)$ is only non-zero provided that $\rho_{a}\left(u_{a}\right)$ and $\bar{v}_{a}$ contain summands that are conjugate to one another. Here, we have written $u \otimes_{\mathbb{C}} \bar{v}=\sum_{a} u_{a} \otimes_{\mathbb{C}} \bar{v}_{a}$ with $u_{a} \otimes_{\mathbb{C}} \bar{v}_{a} \in \mathcal{P}(a) \otimes_{\mathbb{C}} \overline{\mathcal{P}}(a)^{*}$.

As regards the bulk two-point function $B(-,-)$, we choose the following convention. Fixing a bulk two-point function is equivalent to giving an isomorphism $\mathcal{H}_{\text {bulk }} \rightarrow \mathcal{H}_{\text {bulk }}^{*}$, which one can then pre-compose with the canonical pairing $\mathcal{H}_{\text {bulk }} \times \mathcal{H}_{\text {bulk }}^{*} \rightarrow \mathbb{C}$. There is no unique choice, but there is a preferred such isomorphism: if we keep the vector space decomposition implicit in (1.19), the conjugation map flips the direction of all arrows, and effectively turns the composition diagram upside down. We pick the isomorphism which maps the level 0 states of $\mathcal{H}_{\text {bulk }}$ to states of $\mathcal{H}_{\text {bulk }}^{*}$ which have components only at level 0 (and not also at levels 2 and 4). With this choice, the bulk two-point function is non-vanishing only for combinations of states at opposite points in the bulk composition series.

Combining these two considerations we can then determine the components of the bulk composition series in which the Ishibashi state has non-trivial components, as we shall now see.

3.3.1. The corner of the Kac table. Let us begin with the Ishibashi states from the sectors $\mathcal{H}_{-1 / 24}$ and $\mathcal{H}_{35 / 24}$. For these sectors, the Ishibashi morphisms are proportional to the identity and the Ishibashi states are just those built upon the highest weight states. We can then normalize the two resulting Ishibashi states $\left.\left|\frac{-1}{24}\right\rangle\right\rangle$ and $\left.\left|\frac{35}{24}\right\rangle\right\rangle$ such that their overlaps are

$\left\langle\left\langle\frac{-1}{24}\left|q^{L_{0}+\bar{L}_{0}}\right| \frac{-1}{24}\right\rangle\right\rangle=\sqrt{3} \chi_{\mathcal{W}\left(\frac{-1}{24}\right)}(q) \quad$ and $\quad\left\langle\left\langle\frac{35}{24}\left|q^{L_{0}+\bar{L}_{0}}\right| \frac{35}{24}\right\rangle\right\rangle=-\sqrt{3} \chi_{\mathcal{W}\left(\frac{35}{24}\right)}(q)$,

in agreement with (1.21).

3.3.2. The edge of the Kac table. For the sectors along the 'edge of the Kac table', let us first work out the non-trivial components of the Ishibashi states. As in the previous sections, we shall do this explicitly in the $\mathcal{H}_{1 / 8}$ sector; the analysis in the other edge sectors is similar. The Ishibashi morphism $b_{\rho^{\text {id }}}$ is non-vanishing on $u \otimes_{\mathbb{C}} \bar{v} \in \mathcal{H}_{1 / 8}$ provided that the components 
$u_{a} \otimes_{\mathbb{C}} \bar{v}_{a}$ are conjugate to one another in $\mathcal{P}(a) \otimes_{\mathbb{C}} \overline{\mathcal{P}}(a)$. If we choose the representatives $u_{a} \otimes_{\mathbb{C}} \bar{v}_{a}$ according to (2.16), then $u_{a}$ lies in the top $\mathcal{W}(a)$ at level 0 in $\mathcal{P}(a)$. In order for $\bar{v}_{a}$ to be conjugate to $u_{a}$ it must then lie in the bottom $\mathcal{W}(a)$, i.e. at level 2 in $\overline{\mathcal{P}}(a)^{*}$. In terms of the analysis of section 2.3.1, it then follows that $u \otimes_{\mathbb{C}} \bar{v}$ must lie in the grey components of the composition series:

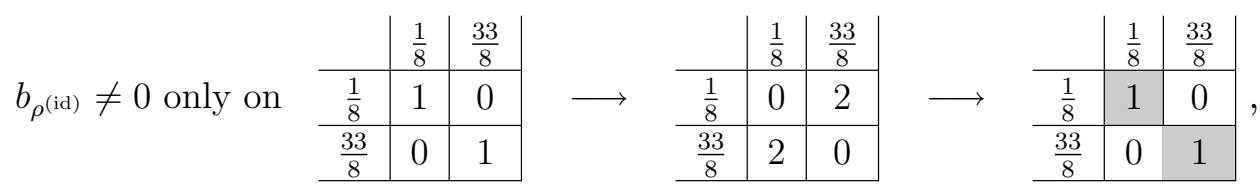

where we have used the same notation as in (1.18). The corresponding Ishibashi state (that we shall denote by $|\mathrm{id}\rangle\rangle$ ) then has non-zero components in the conjugate sectors:

\begin{tabular}{|c|c|c|c|c|c|c|c|c|c|c|c|}
\hline \multirow{3}{*}{$|\mathrm{id}\rangle\rangle \in$} & & $\frac{1}{8}$ & $\frac{33}{8}$ & \multirow{3}{*}{-} & & $\frac{1}{8}$ & $\frac{33}{8}$ & \multirow{3}{*}{$\longrightarrow$} & & $\frac{1}{8}$ & $\frac{33}{8}$ \\
\hline & $\frac{1}{8}$ & 1 & 0 & & $\frac{1}{8}$ & 0 & 2 & & $\frac{1}{8}$ & 1 & 0 \\
\hline & $\frac{33}{8}$ & 0 & 1 & & $\frac{33}{8}$ & 2 & 0 & & $\frac{33}{8}$ & 0 & 1 \\
\hline
\end{tabular}

The analysis for the Ishibashi morphism $\rho^{(a, 2)}$ is similar. Again, $\rho^{(a, 2)}$ is non-vanishing on $u \otimes_{\mathbb{C}} \bar{v} \in \mathcal{H}_{1 / 8}$ provided that $n_{a}(u)$ is conjugate to $\bar{v}$. Since $n_{a}(u)$ maps the top $\mathcal{W}(a)$ to the bottom $\mathcal{W}(a)$ in $\mathcal{P}(a)$, it follows that $u \otimes_{\mathbb{C}} \bar{v}$ has to have non-zero components in the sectors

$b_{\rho^{(1 / 8,2)}} \neq 0$ only on

\begin{tabular}{c|c|c|} 
& $\frac{1}{8}$ & $\frac{33}{8}$ \\
\hline$\frac{1}{8}$ & 1 & 0 \\
\hline$\frac{33}{8}$ & 0 & 1
\end{tabular}$\longrightarrow$\begin{tabular}{c|c|c|} 
& $\frac{1}{8}$ & $\frac{33}{8}$ \\
\hline$\frac{1}{8}$ & 0 & 2 \\
\hline$\frac{33}{8}$ & 2 & 0 \\
\hline
\end{tabular}$\longrightarrow$\begin{tabular}{c|c|c|} 
\\
\hline$\frac{1}{8}$ & 1 & 0 \\
\hline$\frac{33}{8}$ & 0 & 1 \\
\hline
\end{tabular}

and

$b_{\rho^{(33 / 8,2)}} \neq 0$ only on
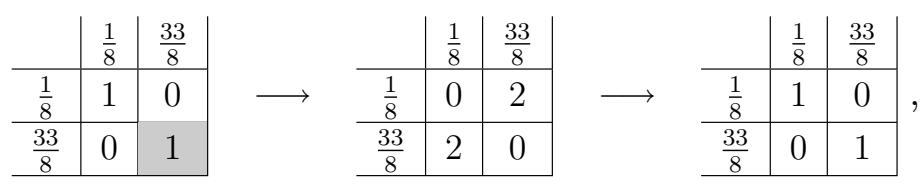

(3.24)

respectively. The corresponding Ishibashi states will be denoted by $\left.\left|n_{1 / 8}\right\rangle\right\rangle$ and $\left.\left|n_{33 / 8}\right\rangle\right\rangle$, and they must have non-trivial components in the conjugate sectors, i.e. in

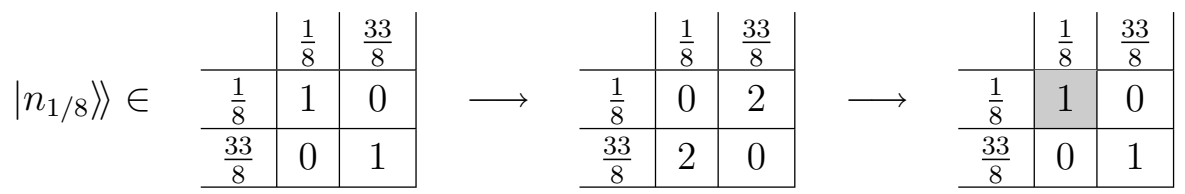

and

\begin{tabular}{|c|c|c|c|c|c|c|c|c|c|c|c|}
\hline \multirow{3}{*}{$\left.\left|n_{33 / 8}\right\rangle\right\rangle \in$} & & $\frac{1}{8}$ & $\frac{33}{8}$ & \multirow{3}{*}{$\longrightarrow$} & & $\frac{1}{8}$ & $\frac{33}{8}$ & \multirow{3}{*}{$\longrightarrow$} & & $\frac{1}{8}$ & $\frac{33}{8}$ \\
\hline & $\frac{1}{8}$ & 1 & 0 & & $\frac{1}{8}$ & 0 & 2 & & $\frac{1}{8}$ & 1 & 0 \\
\hline & $\frac{33}{8}$ & 0 & 1 & & $\frac{33}{8}$ & 2 & 0 & & $\frac{33}{8}$ & 0 & 1 \\
\hline
\end{tabular}


Given this information, we can then directly work out the non-trivial overlaps between these Ishibashi states. For example, for the overlaps of $\left.\left|n_{1 / 8}\right\rangle\right\rangle$ (or $\left.\left|n_{33 / 8}\right\rangle\right\rangle$ ) with themselves vanish, since both of these states only have a component in the 'bottom' factor, but none in the conjugate 'top' factor. On the other hand, the overlap with $|\mathrm{id}\rangle\rangle$ is non-zero, and we can choose the normalization of $\left.\left|n_{1 / 8}\right\rangle\right\rangle$ and $\left.\left|n_{33 / 8}\right\rangle\right\rangle$ such that

$$
\left\langle\left\langle\operatorname{id}\left|q^{L_{0}+\bar{L}_{0}}\right| n_{1 / 8}\right\rangle\right\rangle=\chi_{\mathcal{W}\left(\frac{1}{8}\right)}(q) \quad\left\langle\left\langle\operatorname{id}\left|q^{L_{0}+\bar{L}_{0}}\right| n_{33 / 8}\right\rangle=\chi_{\mathcal{W}\left(\frac{33}{8}\right)}(q) .\right.
$$

Let us decompose the action of $L_{0}$ on a representation $\mathcal{R}$ as $L_{0}=L_{0}^{\text {diag }}+L_{0}^{\text {nil }}$, where $L_{0}^{\text {nil }}$ denotes the nilpotent part. Since for a mode $W_{m}$ of a homogeneous generator $W$ of $\mathcal{W}_{2,3}$ we have $\left[L_{0}, W_{m}\right]=-m W_{m}$ as well as $\left[L_{0}^{\text {diag }}, W_{m}\right]=-m W_{m}$ (after all, $L_{0}^{\text {diag }}$ just gives the grading of $\mathcal{R}$ and $W_{m}$ is a map of degree $-m$ ), it follows that $\left[L_{0}^{\text {nil }}, W_{m}\right]=0$, i.e. the nilpotent part of $L_{0}$ is an intertwiner from $\mathcal{R}$ to itself. In particular, if $\left.|i\rangle\right\rangle$ is an Ishibashi state, so is $\left.\left(L_{0}^{\text {nil }}+\bar{L}_{0}^{\text {nil }}\right)^{n}|i\rangle\right\rangle$ for any $n \geqslant 0$.

The above discussion together with the observations in (3.22), (3.25) and (3.26) shows that $\left.\left(L_{0}^{\text {nil }}+\bar{L}_{0}^{\text {nil }}\right)|\mathrm{id}\rangle\right\rangle$ is again an Ishibashi state, and it has to be a linear combination of $\left.\left|n_{1 / 8}\right\rangle\right\rangle$ and $\left.\left|n_{33 / 8}\right\rangle\right\rangle$. We can choose the normalization of $\left.|\mathrm{id}\rangle\right\rangle$ such that

$$
\left.\left.\left.\frac{1}{2 \pi}\left(L_{0}^{\mathrm{nil}}+\bar{L}_{0}^{\mathrm{nil}}\right)|\mathrm{id}\rangle\right\rangle=\left|n_{1 / 8}\right\rangle\right\rangle+\alpha_{33 / 8}\left|n_{33 / 8}\right\rangle\right\rangle
$$

for some constant $\alpha_{33 / 8} \in \mathbb{C}$. We should note that $\alpha_{33 / 8}$ is in principle determined by the structure of $\mathcal{H}_{\text {bulk }}$, but that with our current limited understanding of the latter we cannot actually calculate it from first principles. In any case, it follows that

$$
\left\langle\left\langle\mathrm{id}\left|q^{L_{0}+\bar{L}_{0}}\right| \mathrm{id}\right\rangle\right\rangle=\mathrm{i} \tau\left(\chi_{\mathcal{W}\left(\frac{1}{8}\right)}(q)+\alpha_{33 / 8} \cdot \chi_{\mathcal{W}\left(\frac{33}{8}\right)}(q)\right) .
$$

If we now set

$\left.\left.\left.\left.\left.\left|\frac{1}{8}, A\right\rangle\right\rangle=\frac{\mathrm{i}}{\sqrt{3}}|\mathrm{id}\rangle\right\rangle, \quad\left|\frac{1}{8}, B\right\rangle\right\rangle=-2 \mathrm{i}\left(\left|n_{1 / 8}\right\rangle\right\rangle+\left|n_{33 / 8}\right\rangle\right\rangle\right), \quad \alpha_{33 / 8}=-2$,

we precisely reproduce the overlaps (1.21).

We should also mention that the two Ishibashi states in (3.30) are characterized by the property that their overlaps do not lead to any $\tilde{\tau}=-1 / \tau$ terms in the open string. Indeed, the additional linear combination, which we could take to be $\left.\left.\left.\left|\frac{1}{8}, C\right\rangle\right\rangle=\left|n_{1 / 8}\right\rangle\right\rangle-\left|n_{33 / 8}\right\rangle\right\rangle$ cannot enter the boundary state construction since it leads, in the open string loop diagram, to a term proportional to $\tilde{\tau}$. For example, we find

$$
\begin{aligned}
\left\langle\left\langle\frac{1}{8}, A\left|q^{L_{0}+\bar{L}_{0}}\right| \frac{1}{8}, C\right\rangle\right\rangle= & \frac{\mathrm{i}}{\sqrt{3}}\left(\chi_{\mathcal{W}\left(\frac{1}{8}\right)}(q)-\chi_{\mathcal{W}\left(\frac{33}{8}\right)}(q)\right) \\
= & -\frac{\mathrm{i}}{18}\left(\chi_{\mathcal{W}\left(\frac{1}{8}\right)}(\tilde{q})+\chi_{\mathcal{W}\left(\frac{33}{8}\right)}(\tilde{q})\right)+\frac{\tilde{\tau}}{3 \sqrt{3}}\left(\chi_{\mathcal{W}\left(\frac{1}{8}\right)}(\tilde{q})-2 \chi_{\mathcal{W}\left(\frac{33}{8}\right)}(\tilde{q})\right) \\
& +(\text { contributions from other sectors }),
\end{aligned}
$$

where $\tilde{\tau}=-1 / \tau$ is the modular parameter in the open string channel. The situation is therefore completely analogous to what was found in [53] for the $\mathcal{W}_{1, p}$-models.

The analysis in the other two sectors is essentially identical. Indeed, the analogues of (3.27) and (3.29) hold also for the Ishibashi states in $\mathcal{H}_{5 / 8}$ and $\mathcal{H}_{1 / 3}$, respectively, and this determines their overlaps up to the constants $\alpha_{21 / 8}$ and $\alpha_{10 / 3}$ as in (3.28). The Ishibashi states of (1.20) can then be identified with

$$
\begin{array}{ll}
\left.\left.\left|\frac{5}{8}, A\right\rangle\right\rangle=\mathrm{i} \sqrt{\frac{2}{3}}|\mathrm{id}\rangle\right\rangle & \left.\left.\left.\left|\frac{5}{8}, B\right\rangle\right\rangle=-\mathrm{i} \sqrt{2}\left(\left|n_{5 / 8}\right\rangle\right\rangle+\left|n_{21 / 8}\right\rangle\right\rangle\right) \\
\left.\left.\left|\frac{1}{3}, A\right\rangle\right\rangle=3^{\frac{1}{4}} \sqrt{2}|\mathrm{id}\rangle\right\rangle & \left.\left.\left.\left|\frac{1}{3}, B\right\rangle\right\rangle=3^{-\frac{3}{4}} \sqrt{2}\left(\left|n_{1 / 3}\right\rangle\right\rangle+\left|n_{10 / 3}\right\rangle\right\rangle\right),
\end{array}
$$


and we reproduce precisely the overlaps (1.21) provided that

$$
\alpha_{21 / 8}=-\frac{1}{2}, \quad \alpha_{10 / 3}=-1
$$

The third Ishibashi state in each of these sectors cannot contribute to the boundary states since it would lead to terms proportional to $\tilde{\tau}$ in the open string channel.

3.3.3. The interior of the Kac table. It therefore only remains to identify the Ishibashi states with components in $\mathcal{H}_{0}$, the 'interior of the Kac table'. The Ishibashi morphism $b_{\rho^{(\mathrm{did})}}$ is nonvanishing for $u \otimes_{\mathbb{C}} \bar{v} \in \mathcal{H}_{0}$ provided that the left and right tensor factors of the representatives in $\hat{\mathcal{H}}_{0}$ are conjugate to one another. By the same logic as above, this can only be the case provided that $u \otimes_{\mathbb{C}} \bar{v}$ lies in the grey components

$b_{\rho^{(\mathrm{id})}} \neq 0$ only on
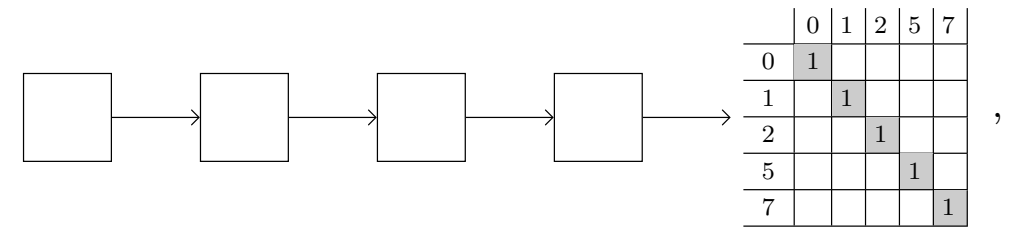

(3.34)

where the empty squares are the remaining composition factors of (1.19), which we have left blank to keep the notation compact. The corresponding Ishibashi state $|\mathrm{id}\rangle\rangle$ therefore has non-vanishing components in the conjugate sectors

$|\mathrm{id}\rangle\rangle \in$

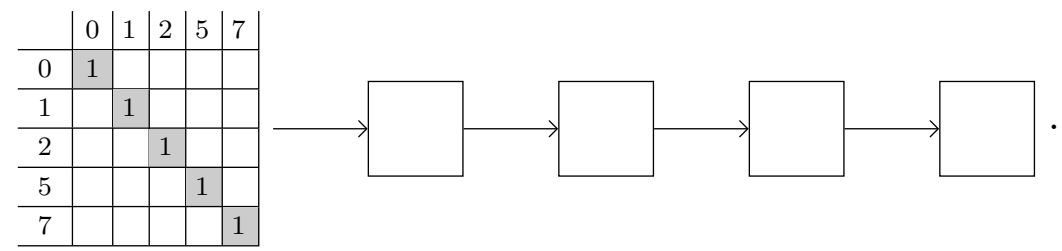

The Ishibashi morphisms $b_{\rho^{\left(\sigma_{i}\right)}}$ are non-vanishing for $u \otimes_{\mathbb{C}} \bar{v} \in \mathcal{H}_{0}$ provided that $\rho^{\left(\sigma_{i}\right)}(u)$ is conjugate to $\bar{v}$, i.e. provided that $u \otimes_{\mathbb{C}} \bar{v}$ lies in the $\mathcal{W}(i) \otimes_{\mathbb{C}} \overline{\mathcal{W}}(i)$ component at level 0 of the $\mathcal{H}_{0}$ composition series, e.g., for $i=2$ :

$b_{\rho^{\left(\sigma_{2}\right)}} \neq 0$ only on

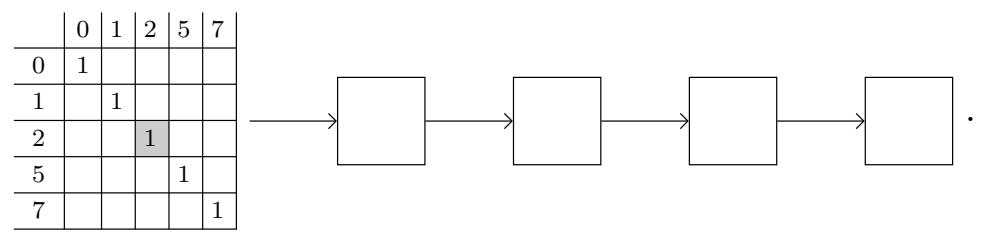

The corresponding Ishibashi state $\left.\left|\sigma_{2}\right\rangle\right\rangle$ must therefore have a non-vanishing component in the conjugate sector:

$\left.\left|\sigma_{2}\right\rangle\right\rangle \in$

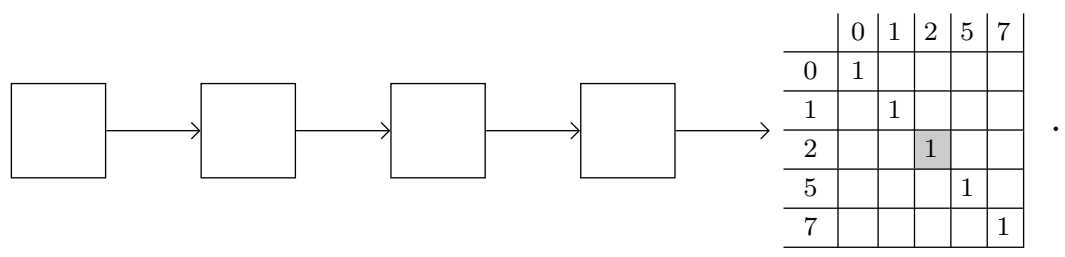


The analysis for the other Ishibashi states $\left.\left|\sigma_{i}\right\rangle\right\rangle$ for $i=0,1,5,7$ is similar, with $\mathcal{W}(2) \otimes_{\mathbb{C}} \overline{\mathcal{W}}(2)$ being replaced by $\mathcal{W}(i) \otimes_{\mathbb{C}} \overline{\mathcal{W}}(i)$.

Finally, we consider the Ishibashi morphisms $b_{\rho^{(\mu)}}, b_{\rho^{(v)}}$ and $b_{\rho^{(\delta)}}$. For them, the analysis is more complicated because of the multiplicities of 2 on the diagonal of the third factor of the composition series (1.19). In (2.16), these two components come from $\mathcal{W}(a) \otimes_{\mathbb{C}} \overline{\mathcal{W}}(a)$, where $\mathcal{W}(a)$ lies at level 0 , while $\overline{\mathcal{W}}(a)$ lies on the very left or very right at level 2 in $\overline{\mathcal{P}}(a)^{*}$; we shall therefore denote these sectors by $\ell$ and $r$, respectively. Note that the sector labelled by $\ell$ is conjugate to that labelled by $r$, and vice versa. With this notation, we have
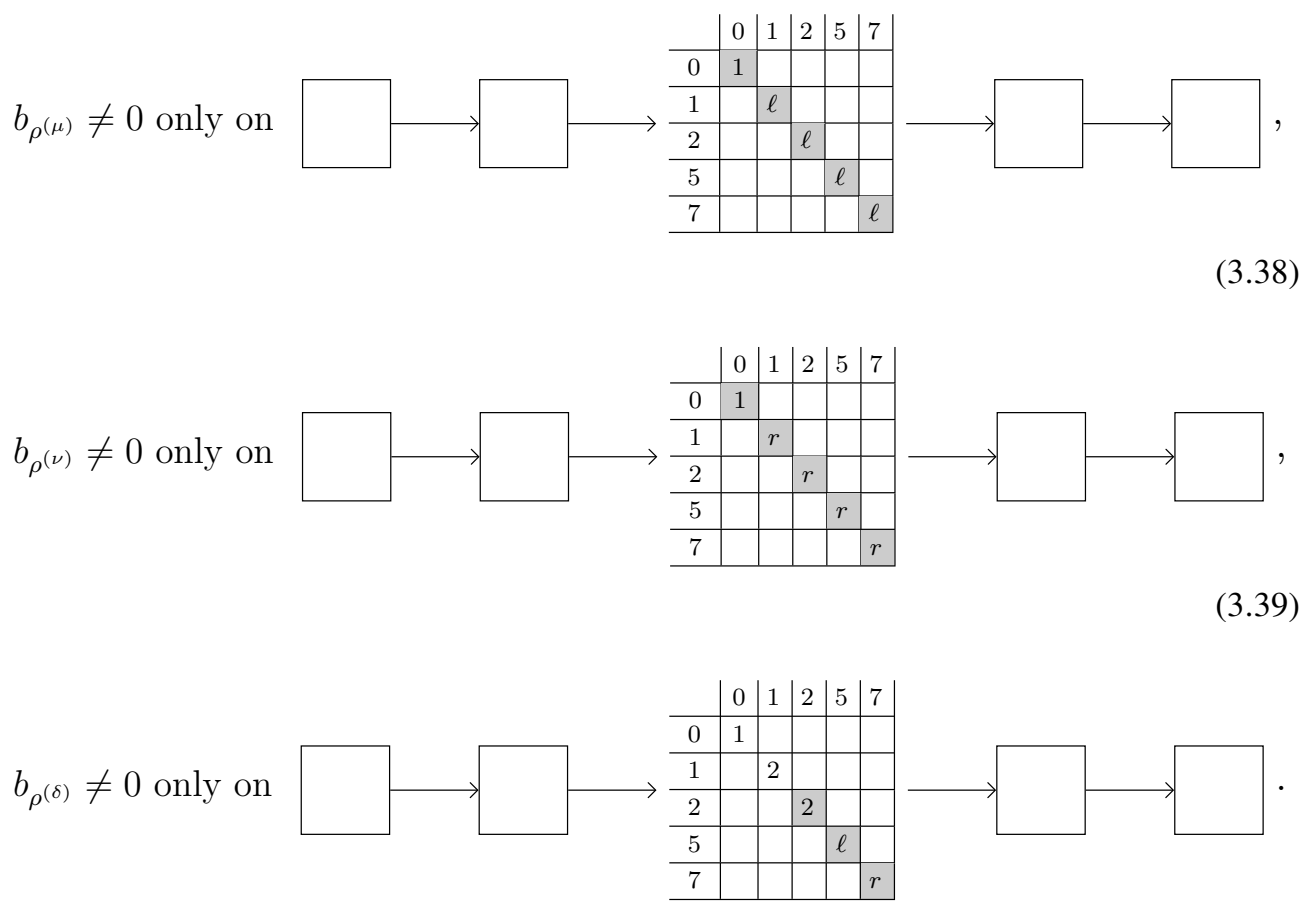

The above diagrams imply in turn that the corresponding Ishibashi states must have nonvanishing components in

$|\mu\rangle\rangle \in$

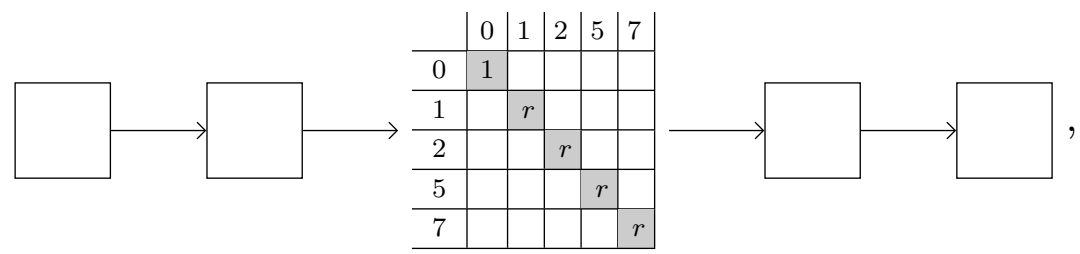

$|\nu\rangle\rangle \in$

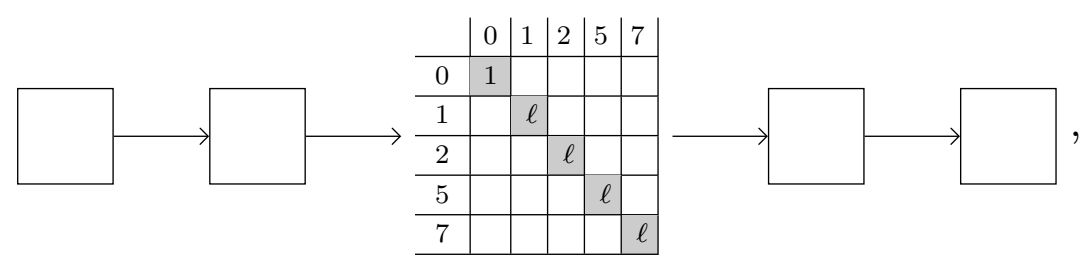




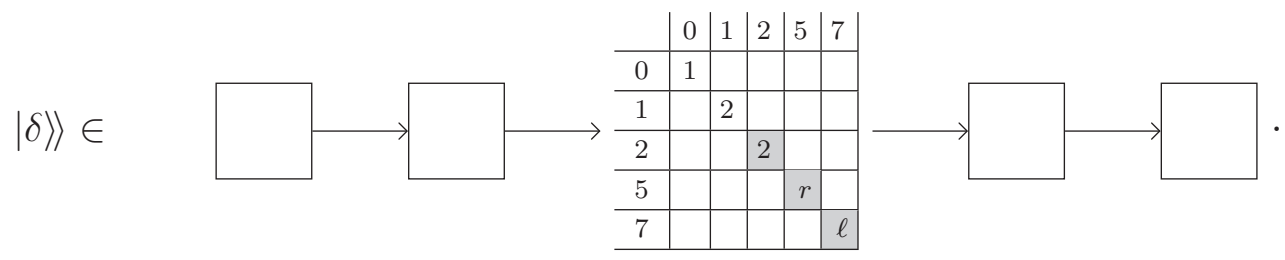

This predicts the following overlaps up to a number of constants that cannot be determined directly in this manner

$$
\begin{aligned}
& \left\langle\left\langle\mathrm{id}\left|q^{L_{0}+\bar{L}_{0}}\right| \mathrm{id}\right\rangle\right\rangle=\tau^{2}\left(\alpha_{0} \chi_{\mathcal{W}(0)}(q)+\alpha_{1} \chi_{\mathcal{W}(1)}(q)+\alpha_{2} \chi_{\mathcal{W}(2)}(q)+\alpha_{5} \chi_{\mathcal{W}(5)}(q)+\alpha_{7} \chi_{\mathcal{W}(7)}(q)\right) \\
& \left\langle\left\langle\operatorname{id}\left|q^{L_{0}+\bar{L}_{0}}\right| \mu\right\rangle\right\rangle=\tau\left(\beta_{0}^{\mu} \chi_{\mathcal{W}(0)}(q)+\beta_{1}^{\mu} \chi_{\mathcal{W}(1)}(q)+\beta_{2}^{\mu} \chi_{\mathcal{W}(2)}(q)+\beta_{5}^{\mu} \chi_{\mathcal{W}(5)}(q)+\beta_{7}^{\mu} \chi_{\mathcal{W}(7)}(q)\right) \\
& \left\langle\left\langle\operatorname{id}\left|q^{L_{0}+\bar{L}_{0}}\right| v\right\rangle\right\rangle=\tau\left(\beta_{0}^{v} \chi_{\mathcal{W}(0)}(q)+\beta_{1}^{v} \chi_{\mathcal{W}(1)}(q)+\beta_{2}^{v} \chi_{\mathcal{W}(2)}(q)+\beta_{5}^{v} \chi_{\mathcal{W}(5)}(q)+\beta_{7}^{v} \chi_{\mathcal{W}(7)}(q)\right) \\
& \left\langle\left\langle\operatorname{id}\left|q^{L_{0}+\bar{L}_{0}}\right| \delta\right\rangle=\tau\left(\beta_{2}^{\delta} \chi_{\mathcal{W}(2)}(q)+\beta_{5}^{\delta} \chi_{\mathcal{W}(5)}(q)+\beta_{7}^{\delta} \chi_{\mathcal{W}(7)}(q)\right)\right. \\
& \left\langle\left\langle\operatorname{id}\left|q^{L_{0}+\bar{L}_{0}}\right| \sigma_{i}\right\rangle\right\rangle=\chi_{\mathcal{W}(i)}(q) \\
& \left\langle\left\langle\mu\left|q^{L_{0}+\bar{L}_{0}}\right| \mu\right\rangle\right\rangle=\alpha^{\mu} \chi_{\mathcal{W}(0)}(q), \quad\left\langle\left\langle v\left|q^{L_{0}+\bar{L}_{0}}\right| v\right\rangle\right\rangle=\alpha^{v} \chi_{\mathcal{W}(0)}(q) \\
& \left\langle\left\langle\delta\left|q^{L_{0}+\bar{L}_{0}}\right| \delta\right\rangle\right\rangle=\alpha^{\delta} \chi_{\mathcal{W}(2)}(q) \\
& \left\langle\left\langle\mu\left|q^{L_{0}+\bar{L}_{0}}\right| v\right\rangle\right\rangle=\gamma_{0}^{\mu v} \chi_{\mathcal{W}(0)}(q)+\gamma_{1}^{\mu v} \chi_{\mathcal{W}(1)}(q)+\gamma_{2}^{\mu v} \chi_{\mathcal{W}(2)}(q)+\gamma_{5}^{\mu v} \chi_{\mathcal{W}(5)}(q)+\gamma_{7}^{\mu v} \chi_{\mathcal{W}(7)}(q) \\
& \left\langle\left\langle\mu\left|q^{L_{0}+\bar{L}_{0}}\right| \delta\right\rangle\right\rangle=\gamma_{2}^{\mu \delta} \chi_{\mathcal{W}(2)}(q)+\gamma_{7}^{\mu \delta} \chi_{\mathcal{W}(7)}(q) \\
& \left\langle\left\langle v\left|q^{L_{0}+\bar{L}_{0}}\right| \delta\right\rangle\right\rangle=\gamma_{2}^{\nu \delta} \chi_{\mathcal{W}(2)}(q)+\gamma_{5}^{v \delta} \chi_{\mathcal{W}(5)}(q) .
\end{aligned}
$$

Some of these constants can be fixed by rescaling the Ishibashi states appropriately. Note that $\left\langle\left\langle\delta\left|q^{L_{0}+\bar{L}_{0}}\right| \delta\right\rangle\right\rangle$ probably vanishes (i.e. $\alpha^{\delta}=0$ ) because the two contributions from the 2's in the middle sector appear to cancel against each other, see (3.17).

Comparing to (1.21), we see that the Ishibashi states that enter the boundary state analysis can be identified with

$$
|0+\rangle\rangle=|\mu\rangle\rangle \quad \text { and } \quad|0-\rangle\rangle=|v\rangle\rangle
$$

provided that $\alpha^{\mu}=\alpha^{\nu}=\frac{1}{2}, \gamma_{0}^{\mu \nu}=\frac{1}{2}$, and $\gamma_{j}^{\mu \nu}=1$ for $j=1,2,5,7$. Thus, we can again obtain all the relevant Ishibashi states within $\mathcal{H}_{\text {bulk }}$.

As before, we can also ask whether there are additional Ishibashi states that could contribute to consistent boundary states. By a straightforward calculation, using the modular transformation properties of appendix A.2, one can check that neither $\mid$ id $\rangle\rangle$ nor $|\delta\rangle\rangle$ can appear in boundary states since $\left\langle\left\langle\mathrm{id}\left|q^{L_{0}+\bar{L}_{0}}\right| \mathrm{id}\right\rangle\right\rangle$ and $\left\langle\left\langle\mu\left|q^{L_{0}+\bar{L}_{0}}\right| \delta\right\rangle\right\rangle$ both lead to $\tilde{\tau}$ terms in the open string loop diagrams. On the other hand, a similar argument does not apply to the Ishibashi states $\left.\left|\sigma_{i}\right\rangle\right\rangle$ since their relative overlaps, as well as their overlaps with $\left.|\mu\rangle\right\rangle$ and $\left.|v\rangle\right\rangle$ all vanish. Thus, these Ishibashi states do not contribute to the cylinder diagrams, and we cannot decide whether they appear in boundary states based on cylinder diagrams alone.

Given that the subgroup $\mathrm{K}_{0}^{b}$ of the Grothendieck group is 12 dimensional but only ten Ishibashi states are required for the description of the cylinder diagrams, we suspect that at least certain linear combinations of the $\left.\left|\sigma_{i}\right\rangle\right\rangle$ also contribute to the boundary states. In fact, this is even required in order for the bulk-boundary map to be non-degenerate in the bulk entry. To see this, we observe that the bulk states from the top level in $\mathcal{H}_{0}$ only contribute in overlaps with the $\left.\left|\sigma_{i}\right\rangle\right\rangle$ Ishibashi states, see in particular equation (3.36). Thus, if the $\left.\left|\sigma_{i}\right\rangle\right\rangle$ Ishibashi did not appear in any of the boundary states, all bulk-boundary maps would vanish on these top 
states, in contradiction with the assumed non-degeneracy. However, since the $\left.\left|\sigma_{i}\right\rangle\right\rangle$ Ishibashi states do not contribute to any cylinder diagrams, the above analysis does not determine their coefficients.

\section{Conclusion}

In this paper, we have made a proposal for the bulk space for the 'charge-conjugation' modular invariant of the logarithmic $\mathcal{W}_{2,3}$-triplet model. The basic idea of our construction is to obtain the bulk space from a given boundary condition as the largest space for which a suitable bulkboundary map is non-degenerate in the bulk entry. Unlike the situation for the logarithmic $\mathcal{W}_{1, p}$-models where the starting point of the analysis was taken to be the 'identity brane' whose space of boundary fields just consists of $\mathcal{W}_{1, p}$, no such brane exists for $\mathcal{W}_{2,3}$. We have therefore not been able to perform this analysis starting from an actual boundary condition. Instead, we have argued that for the purpose of identifying the 'charge-conjugation' bulk spectrum we may take the space of boundary fields to consist just of $\mathcal{W}^{*}$, the representation conjugate to $\mathcal{W}$. With this assumption the analysis could then be performed in a similar way to what was done for the $\mathcal{W}_{1, p}$-models. The resulting bulk space satisfies a number of non-trivial consistency conditions: the space of bulk states is self-conjugate (as has to be the case in order for the bulk two-point function to be non-degenerate), the partition function is modular invariant, and we could identify boundary states whose annulus partition functions reproduce the results of [41]. None of these consistency conditions are part of our ansatz, and we therefore regard these tests as very good evidence for the correctness of our proposal.

The resulting bulk space could be written as a quotient of the direct sum of tensor products of the projective covers of the irreducible representations. Furthermore, the representatives of the quotient space could be taken to be described by the direct sum of tensor products of each irreducible representation with its projective cover. The bulk spectrum has therefore exactly the same structure as for the $\mathcal{W}_{1, p}$-models [53], or the supergroup models of [28]. It is plausible that the 'charge-conjugation' theory of a generic (logarithmic) conformal field theory may therefore have this structure.

On the other hand, there are also important differences to what was found for the $\mathcal{W}_{1, p^{-}}$ models. One surprising property is that the bulk space does not contain $\mathcal{W}_{2,3} \otimes_{\mathbb{C}} \overline{\mathcal{W}}_{2,3}$ as a sub-representation, but only as a sub-quotient. The other unusual feature is that there are non-trivial Ishibashi states that do not contribute to annulus amplitudes. As a consequence, the boundary states of the various boundary conditions could not be fixed uniquely by these amplitudes alone. It would be interesting to see whether there are other (easily accessible) amplitudes that would allow one to determine the boundary states uniquely. One of us has recently studied the action of topological defects on these boundary states [62], but, unfortunately, this does not seem to constrain the boundary states any further. (However, it is encouraging that the defect analysis also seems to work nicely for this theory.)

On a technical level, probably the most tricky part of our analysis was the description of the projective covers of all irreducible representations, see appendix A.1 and in particular appendix B. Some aspects of the corresponding composition series could be deduced from the conjectures of [9], while for the determination of the projective cover $\mathcal{P}(0)$ of the trivial representation $\mathcal{W}(0)$ we had to use other arguments, in particular the recent calculation of Zhu's algebra [46]. The fact that everything fits together nicely makes us confident that the composition series of these projective covers are indeed correct, but a direct confirmation, for example by using the Coulomb gas description of [44, 63], is still missing. 
As far as we know, the proposal of this paper is the first example of an interesting 9 (seemingly) consistent bulk theory with a discrete spectrum at $c=0$ (there are earlier examples with continuous spectrum, see [27]). It would therefore be very instructive to study its properties further. For example, it would be interesting to determine the relevant critical exponents, and to understand which correlators are logarithmic, etc. In particular, this may give some insight into the structure of closely related bulk theories, such as those describing polymers or percolation.

\section{Acknowledgments}

We thank Alexei Davydov, Jürgen Fuchs, Azat Gainutdinov, Volker Schomerus, Christoph Schweigert and Akihiro Tsuchiya for useful conversations. The research of MRG and SW is partially supported by a grant from the Swiss National Science Foundation. The work of IR is supported by the German Science Foundation (DFG) within the Collaborative Research Center 676 'Particles, Strings and the Early Universe'. MRG and IR thank the ErwinSchrödinger-Institute in Vienna for hospitality during the workshop 'Quantum field theory in curved spacetimes and target-spaces', where part of this work was completed.

\section{Appendix A. $\mathcal{W}_{2,3}$-representations}

\section{A.1. Composition series}

Here, we list the embedding diagrams for the projective covers $\mathcal{P}(0), \mathcal{P}(1)=\mathcal{R}^{(3)}(0,0,1,1)$, $\mathcal{P}(2)=\mathcal{R}^{(3)}(0,0,2,2), \mathcal{P}(5)=\mathcal{R}^{(3)}(0,1,2,5)$ and $\mathcal{P}(7)=\mathcal{R}^{(3)}(0,1,2,7)$. The structure of $\mathcal{P}(0)$ is explained in appendix $\mathrm{B}$, while the structure of the other projective covers was deduced from the conjectures of [9] and from the analogy with quantum group representations described in [35, section 6.1] and [37, section 2.2].

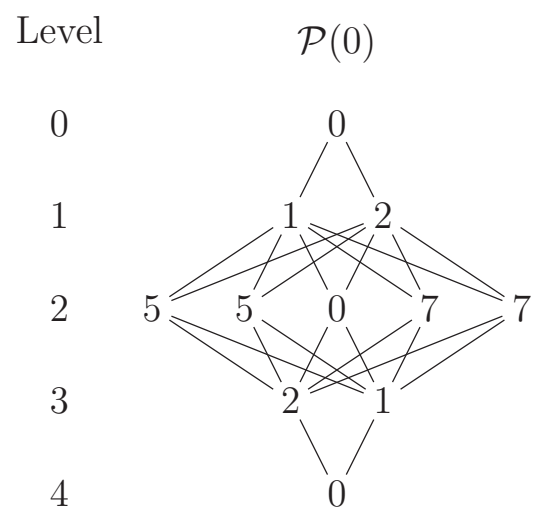

\footnotetext{
9 Here, 'interesting' refers to the fact that the theory is not just the tensor product of rational theories whose total central charge adds up to zero.
} 


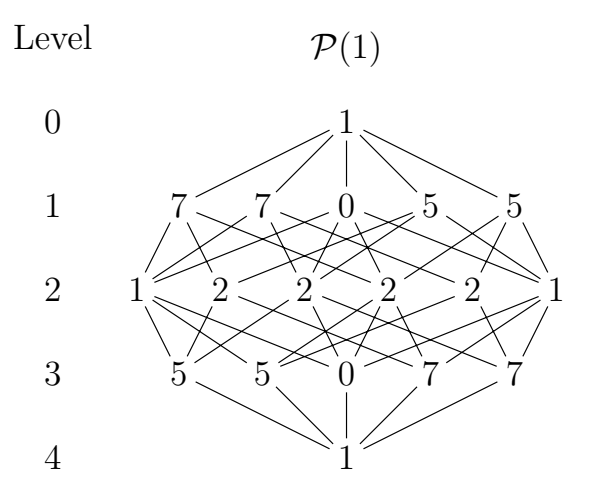

Level

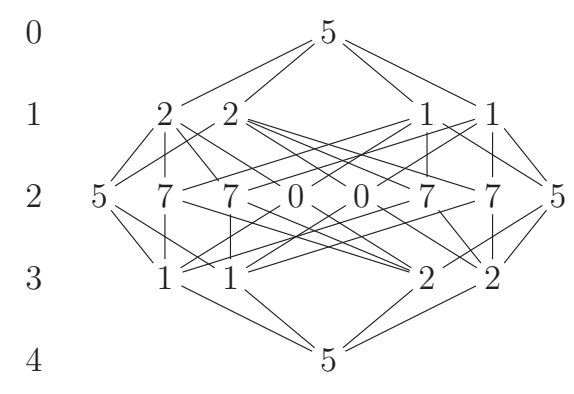

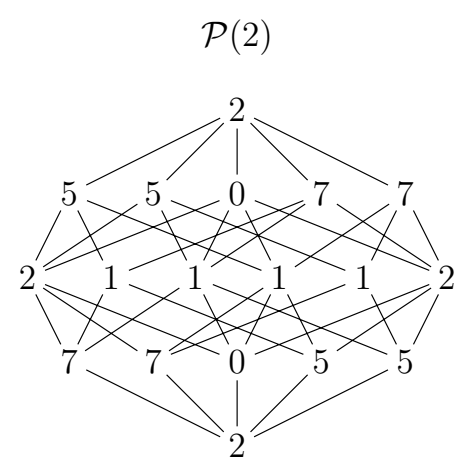

Looking at the embedding diagrams from top to bottom, we say that the representations at the top are at level zero, the representations in the second line from the top are at level one, etc. (This is indicated in the above diagrams by the small number written on the left.)

Let us explain the meaning of the lines in these diagrams by taking $\mathcal{P}(2)$ as an example. The embedding diagram for $\mathcal{P}(2)$ states that one can choose a direct sum decomposition of $\mathcal{P}(2)$ as a vector space as

$$
\mathcal{P}(2) \cong 2 \mathcal{W}(0) \oplus 4 \mathcal{W}(1) \oplus 4 \mathcal{W}(2) \oplus 4 \mathcal{W}(5) \oplus 4 \mathcal{W}(7)
$$

Furthermore, the action of a mode in $\mathcal{W}_{2,3}$ on a state in $\mathcal{W}(h)$ gives a vector in the direct sum of the given $\mathcal{W}(h)$ and all summands joined to it by downward directed lines (in one or more steps). In particular, these diagrams are therefore in general not unique. For example, there must also exist a different direct sum decomposition of $\mathcal{P}(2)$ as in (A.4) such that $\mathcal{W}(0)$ at level 1 is joined only to one $\mathcal{W}(1)$ and one $\mathcal{W}(2)$ at level 2 . (This has to be possible for there to be an intertwiner $\mathcal{P}(0) \rightarrow \mathcal{P}(2)$ whose image contains $\mathcal{W}(0) \subset \mathcal{P}(2)$ at level 1.)

For completeness, we also give the embedding diagrams for the other indecomposable representations that appear in our analysis:

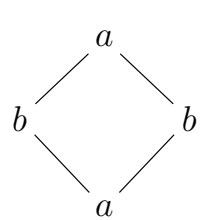

$$
\begin{array}{rlll}
\mathcal{R}^{(2)}\left(\frac{1}{3}, \frac{1}{3}\right): & a=\frac{1}{3}, b=\frac{10}{3} & \mathcal{R}^{(2)}(2,7): & a=7, b=2 \\
\mathcal{R}^{(2)}\left(\frac{1}{3}, \frac{10}{3}\right): & a=\frac{10}{3}, b=\frac{1}{3} & \mathcal{R}^{(2)}(2,5): & a=5, b=2 \\
\mathcal{R}^{(2)}\left(\frac{5}{8}, \frac{5}{8}\right): & a=\frac{5}{8}, b=\frac{21}{8} & \mathcal{R}^{(2)}(1,7): & a=7, b=1 \\
\mathcal{R}^{(2)}\left(\frac{5}{8}, \frac{21}{8}\right): & a=\frac{21}{8}, b=\frac{5}{8} & \mathcal{R}^{(2)}(1,5): & a=5, b=1 \\
\mathcal{R}^{(2)}\left(\frac{1}{8}, \frac{1}{8}\right): & a=\frac{1}{8}, b=\frac{33}{8} & & \\
\mathcal{R}^{(2)}\left(\frac{1}{8}, \frac{33}{8}\right): & a=\frac{33}{8}, b=\frac{1}{8} & &
\end{array}
$$


and<smiles>O1P2O[Te]12</smiles>

$$
\begin{array}{ll}
\mathcal{R}^{(2)}(0,2)_{7}: & a=2, b=7 \\
\mathcal{R}^{(2)}(0,2)_{5}: & a=2, b=5 \\
\mathcal{R}^{(2)}(0,1)_{7}: & a=1, b=7 \\
\mathcal{R}^{(2)}(0,1)_{5}: & a=1, b=5
\end{array}
$$

For the analysis of the Ishibashi states, we also need to introduce some notation for the various intertwiners. We say an intertwiner $e: \mathcal{P}(a) \rightarrow \mathcal{P}(b)$ has degree $l$ if it adds $l$ to the level of the states, i.e. if it maps the representation $\mathcal{W}(a)$ at level zero to a representation at level $l$ in $\mathcal{P}(b)$. We denote such an intertwiner by $e_{l}^{a \rightarrow b}$. Sometimes, this information does not specify an intertwiner uniquely; for example, there are two intertwiners of degree 1 corresponding to $e_{1}^{1 \rightarrow 5}$. Whenever an intertwiner is not uniquely specified in this manner, we introduce an additional label, i.e. we write $e_{1 ; \alpha}^{1 \rightarrow 5}$ where $\alpha$ takes two values. In the present context, it is natural to take $\alpha \in\{L, R\}$, where $e_{1 ; R}^{1 \rightarrow 5}$ is the intertwiner that maps the circled nodes in the following diagram to one another:

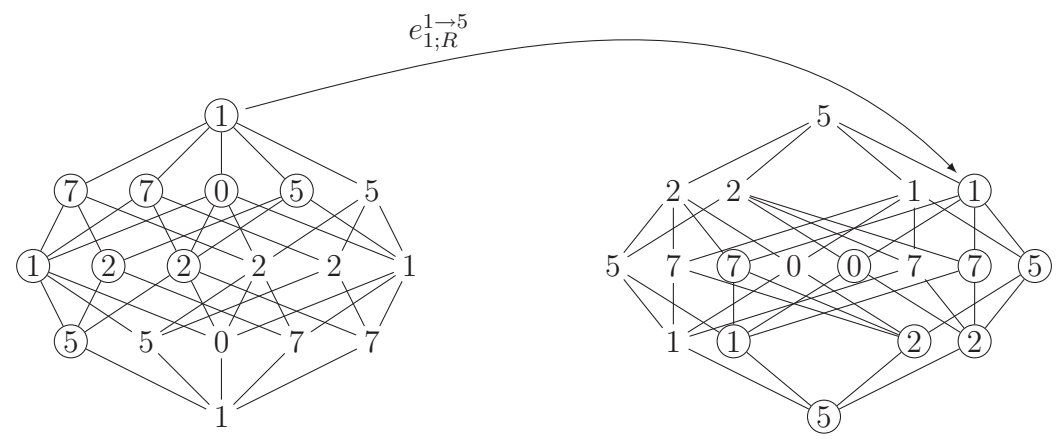

(A.7)

and similarly for $e_{1 ; L}^{1 \rightarrow 5}$. For the case of the intertwiners from $\mathcal{P}(h) \rightarrow \mathcal{P}(h)$ with $h \in\{1,2,5,7\}$, it is convenient to label the different intertwiners at level 2 by the representations to which the image node at level 2 is connected, e.g., the intertwiner $e_{2 ; 5}^{1 \rightarrow 1}$ maps the circled nodes to one another, etc.

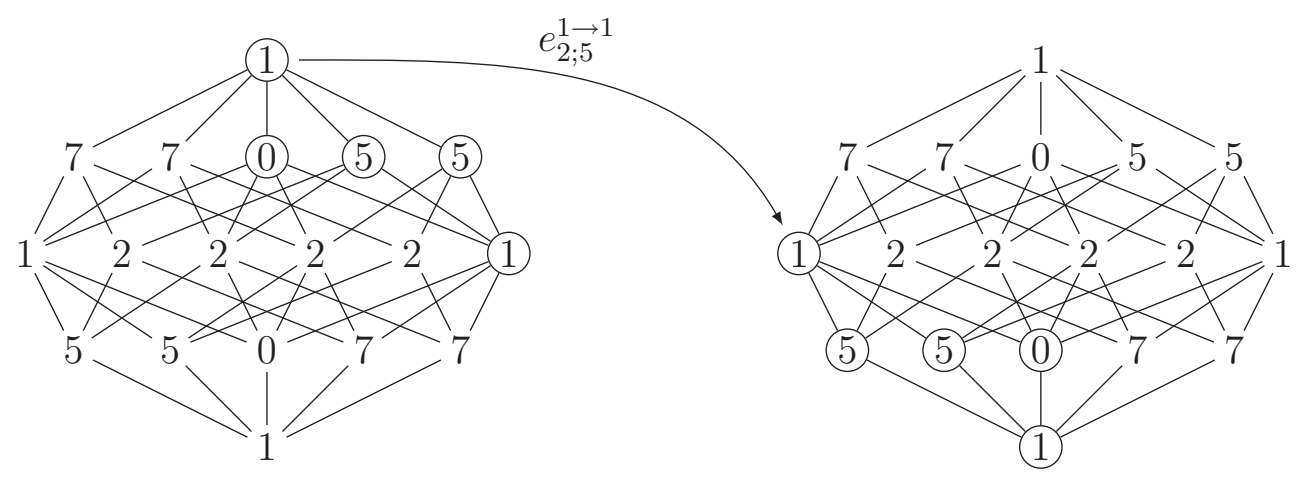

(A.8)

These diagrams then allow one to compute the composition of intertwiners. For example, we have $e_{1 ; R}^{1 \rightarrow 5} \circ e_{2 ; 5}^{1 \rightarrow 1}=e_{3 ; R}^{1 \rightarrow 5}$, where $e_{3 ; R}^{1 \rightarrow 5}$ maps the top $\mathcal{W}(1)$ of $\mathcal{P}(1)$ to the 'right' $\mathcal{W}(1)$ at level 3 of $\mathcal{P}(5)$. By the same reasoning, $e_{1 ; L / R}^{1 \rightarrow 7} \circ e_{2 ; 5}^{1 \rightarrow 1}=0$. 


\section{A.2. Modular transformations}

In this appendix, we briefly review the modular $S$-matrix of the $\mathcal{W}_{2,3}$-model following [38]. ${ }^{10}$ To keep the notation for the $S$-modular transformation of the characters of all irreducible representations compact, we will label each character by the position of its corresponding irreducible representation in the Kac table (1.4). Because each cell of the Kac table contains two or three entries, we will denote the character of the right most irreducible representation in the cell by $\chi_{(r, s,-)}$ and the second to right representations $\chi_{(r, s,+)}$. The two cells in the interior of the Kac table both contain an additional weight 0 representation; this is just $\mathcal{W}(0)$ and we will continue to refer to the character of $\mathcal{W}(0)$ by $\chi_{\mathcal{W}}(0)$.

The $S$-modular transformation of the characters is then, for $\varepsilon= \pm$,

$\chi_{\mathcal{W}(0)}\left(-\frac{1}{\tau}\right)=\chi_{\mathcal{W}(0)}(\tau)$

$\chi_{(\rho, \sigma, \varepsilon)}\left(-\frac{1}{\tau}\right)=\widetilde{\mathcal{S}}_{\rho, \sigma}^{\varepsilon}(\tau) \chi_{\mathcal{W}(0)}(\tau)+\sum_{r=1}^{2} \sum_{s=1}^{3} \varepsilon^{r} \mathcal{S}_{\rho, \sigma ; r, s}(\tau)\left(\chi_{(r, s,+)}(\tau)+(-1)^{\rho} \chi_{(r, s,-)}(\tau)\right)$

where $\rho \in\{1,2\}, \sigma \in\{1,2,3\}$ and the $\tau$-dependant $S$-matrix coefficients are given by

$$
\begin{aligned}
& \mathcal{S}_{\rho, \sigma ; 1, s}(\tau)= \frac{1}{3 \sqrt{3}}(-1)^{\rho s+\sigma}\left(\rho \cos \frac{\pi 3 \rho}{2}-\mathrm{i} \tau \sin \frac{\pi 3 \rho}{2}\right) \\
& \times\left(\sigma \cos \frac{\pi 2 \sigma s}{3}-\mathrm{i} \tau(3-s) \sin \frac{\pi 2 \sigma s}{3}\right), \quad s \in\{1,2\} \\
& \mathcal{S}_{\rho, \sigma ; 1,3}(\tau)= \frac{\sigma}{6 \sqrt{3}}(-1)^{\sigma+\rho}\left(\rho \cos \frac{\pi 3 \rho}{2}-\mathrm{i} \tau \sin \frac{\pi 3 \rho}{2}\right), \\
& \mathcal{S}_{\rho, \sigma ; 2, s}(\tau)= \frac{\rho}{6 \sqrt{3}}(-1)^{(s+1) \rho}\left(\sigma \cos \frac{\pi 2 \sigma s}{3}-\mathrm{i} \tau(3-s) \sin \frac{\pi 2 \sigma s}{3}\right), \quad s \in\{1,2\} \\
& \mathcal{S}_{\rho, \sigma ; 2,3}= \frac{\rho \sigma}{12 \sqrt{3}} \\
& \widetilde{\mathcal{S}}_{\rho, \sigma}^{+}(\tau)=(-1)^{\rho+\sigma} \frac{1}{36 \sqrt{3}}\left(6 \rho \sigma \cos \frac{\pi 3 \rho}{2} \cos \frac{\pi 2 \sigma}{3}-i 3 \rho \tau \cos \frac{\pi 3 \rho}{2} \sin \frac{\pi 2 \sigma}{3}\right. \\
&\left.\quad+\mathrm{i} 2 \sigma \tau \cos \frac{\pi 2 \sigma}{3} \sin \frac{\pi 3 \rho}{2}+\left(\frac{1}{2} \tau^{2}-\frac{\mathrm{i} 3 \tau}{\pi}+\frac{4 \sigma^{2}+9 \rho^{2}}{2}\right) \sin \frac{\pi 3 \rho}{2} \sin \frac{\pi 2 \sigma}{3}\right) \\
& \widetilde{\mathcal{S}}_{\rho, \sigma}^{-}=-\widetilde{\mathcal{S}}_{\rho, \sigma}^{+}(\tau)+(-1)^{\rho+\sigma} \frac{1}{\sqrt{12}} \sin \frac{\pi 3 \rho}{2} \sin \frac{\pi 2 \sigma}{3} .
\end{aligned}
$$

\section{Appendix B. Projective cover of $\mathcal{W}(0)$}

Given the results of [55, theorem 3.24] it seems plausible that $\mathcal{W}(0)$ must have a projective cover $\mathcal{P}(0)$. In this appendix, we want to deduce the structure of $\mathcal{P}(0)$, assuming its existence, and using input from a recent calculation of Zhu's algebra [46]. For the following, it will be convenient to introduce the abbreviation

$$
\langle U, V\rangle=\operatorname{dim} \operatorname{Hom}(U, V),
$$

where $U$ and $V$ are two $\mathcal{W}$-representations. As was argued in [41, section 3.1], we have isomorphisms $\pi_{U, V}: \operatorname{Hom}\left(U, V^{*}\right) \rightarrow \operatorname{Hom}\left(U \otimes_{f} V, \mathcal{W}^{*}\right)$ that are natural in $U$ and $V$ and thus

$$
\left\langle U, V^{*}\right\rangle=\left\langle U \otimes_{f} V, \mathcal{W}^{*}\right\rangle .
$$

${ }^{10}$ There are two small typos in [38] (arXiv v2): in (2.5) there should be an additional $(-1)^{p p^{\prime}}$ in front of $\chi_{s, s^{\prime}}^{-}$, and in the expression for $\widetilde{\mathcal{S}}_{r, r^{\prime} ; s, s^{\prime}}^{+}(\tau)$, the summand $-2 \mathrm{i} \pi p p^{\prime} \tau$ should read $-\mathrm{i} p p^{\prime} \tau /(2 \pi)$. 
Finally, we note that $\operatorname{dim} \operatorname{Hom}(\mathcal{P}(h), U)$ gives the number of occurrences of $\mathcal{W}(h)$ in the composition series of $U$, i.e.

$$
\langle\mathcal{P}(h), U\rangle=(\text { number of } \mathcal{W}(h) \text { in comp. series of } U) .
$$

\section{B.1. Fusion rules}

Even without knowledge of the structure of $\mathcal{P}(0)$, we can partially deduce its fusion rules.

Fusion with $\mathcal{W}(0) . \quad$ It was argued in [41, section 2], the fusion of $\mathcal{W}(0)$ with any $\mathcal{W}$ representation can only produce multiples of $\mathcal{W}(0)$. Thus, $\mathcal{P}(0) \otimes_{f} \mathcal{W}(0) \cong n \mathcal{W}(0)$ for some $n \geqslant 0$. Because of $\operatorname{dim} \operatorname{Hom}(\mathcal{P}(0), \mathcal{W}(0))=1$, it follows from (B.2) and (B.3) that

$$
1=\langle\mathcal{P}(0), \mathcal{W}(0)\rangle=\left\langle\mathcal{P}(0) \otimes_{f} \mathcal{W}(0), \mathcal{W}^{*}\right\rangle=n\left\langle\mathcal{W}(0), \mathcal{W}^{*}\right\rangle=n
$$

Thus, we conclude that

$$
\mathcal{P}(0) \otimes_{f} \mathcal{W}(0) \cong \mathcal{W}(0) .
$$

Fusion with representations $U$ that have a dual. Given an object $U$ in a monoidal category $\mathcal{C}$, we call an object $U^{\vee} \in \mathcal{C}$ a (left and right) dual of $U$ iff there are intertwiners

$$
\begin{array}{ll}
b_{U}: \mathbf{1} \rightarrow U \otimes_{f} U^{\vee}, & \tilde{b}_{U}: \mathbf{1} \rightarrow U^{\vee} \otimes_{f} U, \\
d_{U}: U^{\vee} \otimes_{f} U \rightarrow \mathbf{1}, & \tilde{d}_{U}: U \otimes_{f} U^{\vee} \rightarrow \mathbf{1},
\end{array}
$$

subject to the usual conditions, see e.g. [66, definition 2.1.1]. We want to deduce the fusion rules of $\mathcal{P}(0)$ with representations that have a dual. Our analysis is based on the following result (see e.g. [67, p 441, corollaries 1 and 2]).

Lemma B.1. Let $\mathcal{C}$ be a monoidal category and $U \in \mathcal{C}$ have (left and right) dual $U^{\vee}$.

(i) If $f: X \rightarrow Y$ is epi, so are $f \otimes \mathrm{id}_{U}$ and $\mathrm{id}_{U} \otimes f$.

(ii) If $P \in \mathcal{C}$ is projective, so are $P \otimes U$ and $U \otimes P$.

Proof. Part (i). We will show that $f \otimes \mathrm{id}_{U}$ is epi; the other case follows analogously. We need to check that for any two morphisms $a, b: Y \otimes U \rightarrow Z, a \circ\left(f \otimes \mathrm{id}_{U}\right)=b \circ\left(f \otimes \mathrm{id}_{U}\right)$ implies $a=b$. For $k: Y \otimes U \rightarrow Z$ define $C(k): Y \rightarrow Z \otimes U^{\vee}$ as (we do not write out associators and unit isomorphisms)

$$
C(k)=\left(k \otimes \mathrm{id}_{U^{\vee}}\right) \circ\left(\mathrm{id}_{Y} \otimes b_{U}\right) .
$$

From the properties of duality morphisms, it is easy to see that $a \circ\left(f \otimes \mathrm{id}_{U}\right)=b \circ\left(f \otimes \mathrm{id}_{U}\right)$ implies $C(a) \circ f=C(b) \circ f$. By assumption, $f$ is epi, so that $C(a)=C(b)$. Again by properties of duality maps, this in turn implies $a=b$.

Part (ii): We will show that $P \otimes U$ is projective, the other case follows analogously. We have to check that for any epi $f: X \rightarrow Y$ and any $h: P \otimes U \rightarrow Y$ there is a $h^{\prime}: P \otimes U \rightarrow X$ such that $h=f \circ h^{\prime}$. Note that since $U$ has left and right duals $U^{\vee}$, the left and right duals of $U^{\vee}$ can be chosen to be $U$. Applying part (i) to $U^{\vee}$ we conclude that $f \otimes \mathrm{id}_{U^{\vee}}$ is epi. Given the map $C(h): P \rightarrow Y \otimes U^{\vee}$, since $P$ is projective there exists a map $q: P \rightarrow X \otimes U^{\vee}$ such that $C(h)=\left(f \otimes \operatorname{id}_{U^{\vee}}\right) \circ q$. The map

$$
h^{\prime}=\left(\mathrm{id}_{X} \otimes d_{U}\right) \circ\left(q \otimes \mathrm{id}_{U}\right)
$$

satisfies $h=f \circ h^{\prime}$, as can again easily be seen using the properties of duality maps. 
The map $C$ used in the above proof also shows that

$$
U \text { has a dual } \Rightarrow\langle A \otimes U, B\rangle=\left\langle A, B \otimes U^{\vee}\right\rangle \text { for all } A, B .
$$

Of the 35 indecomposable representations listed in (1.4) and (1.5), all representations not in grey boxes have a dual, as do $\mathcal{W}$ and $\mathcal{Q}$. Let us collect these 28 representations in a set $\mathcal{D}$. By lemma appendix B.1, for any $U \in \mathcal{D}, \mathcal{P}(0) \otimes_{f} U$ is projective, that is, we can write

$$
\mathcal{P}(0) \otimes_{f} U \cong \bigoplus_{h} n_{h}(U) \mathcal{P}(h),
$$

where the sum runs over the 13 values that $h$ takes for the irreducible representations $\mathcal{W}(h)$ according to (1.4). The multiplicities $n_{h}(U)$ are obtained via

$$
n_{h}(U)=\left\langle\mathcal{P}(0) \otimes_{f} U, \mathcal{W}(h)\right\rangle=\left\langle\mathcal{P}(0), \mathcal{W}(h) \otimes_{f} U^{\vee}\right\rangle,
$$

where the right-hand side can be computed form the fusion rules in [41, appendix A.4] and the composition series given appendix A.1. Since $\mathcal{W}(0) \otimes_{f} U^{\vee}=0$ for all $U \in \mathcal{D}$, we conclude $n_{0}(U)=0$. The other multiplicities are obtained case by case, for example

$$
n_{1}\left(\mathcal{W}\left(\frac{1}{3}\right)\right)=\left\langle\mathcal{P}(0), \mathcal{W}(1) \otimes_{f} \mathcal{W}\left(\frac{1}{3}\right)\right\rangle=\left\langle\mathcal{P}(0), \mathcal{R}^{(2)}(0,1)_{7}\right\rangle=1
$$

Altogether, this gives

$$
\begin{aligned}
& \mathcal{W}\left(\frac{1}{3}\right) \otimes_{f} \mathcal{P}(0)=\mathcal{P}\left(\frac{1}{3}\right) \oplus \mathcal{P}(1) \\
& \mathcal{W}\left(\frac{10}{3}\right) \otimes_{f} \mathcal{P}(0)=\mathcal{P}\left(\frac{10}{3}\right) \oplus \mathcal{P}(5) \\
& \mathcal{W}\left(\frac{5}{8}\right) \otimes_{f} \mathcal{P}(0)=\mathcal{P}\left(\frac{1}{8}\right) \oplus \mathcal{P}\left(\frac{5}{8}\right) \\
& \mathcal{W}\left(\frac{33}{8}\right) \otimes_{f} \mathcal{P}(0)=\mathcal{P}\left(\frac{33}{8}\right) \oplus \mathcal{P}\left(\frac{21}{8}\right) \\
& \mathcal{W}\left(\frac{1}{8}\right) \otimes_{f} \mathcal{P}(0)=\mathcal{P}\left(\frac{1}{8}\right) \oplus \mathcal{P}\left(\frac{5}{8}\right) \oplus 2 \mathcal{W}\left(\frac{-1}{24}\right) \oplus 2 \mathcal{W}\left(\frac{35}{24}\right) \\
& \mathcal{W}\left(\frac{21}{8}\right) \otimes_{f} \mathcal{P}(0)=\mathcal{P}\left(\frac{33}{8}\right) \oplus \mathcal{P}\left(\frac{21}{8}\right) \oplus 2 \mathcal{W}\left(\frac{-1}{24}\right) \oplus 2 \mathcal{W}\left(\frac{35}{24}\right) \\
& \mathcal{W}\left(\frac{-1}{24}\right) \otimes_{f} \mathcal{P}(0)=2 \mathcal{P}\left(\frac{1}{8}\right) \oplus 2 \mathcal{P}\left(\frac{21}{8}\right) \oplus 2 \mathcal{W}\left(\frac{-1}{24}\right) \oplus 2 \mathcal{W}\left(\frac{35}{24}\right) \\
& \mathcal{W}\left(\frac{35}{24}\right) \otimes_{f} \mathcal{P}(0)=2 \mathcal{P}\left(\frac{1}{8}\right) \oplus 2 \mathcal{P}\left(\frac{21}{8}\right) \oplus 2 \mathcal{W}\left(\frac{-1}{24}\right) \oplus 2 \mathcal{W}\left(\frac{35}{24}\right) \\
& \mathcal{R}^{(2)}(0,2)_{7} \otimes_{f} \mathcal{P}(0)=\mathcal{P}(1) \oplus \mathcal{P}(2) \\
& \mathcal{R}^{(2)}(2,7) \otimes_{f} \mathcal{P}(0)=\mathcal{P}(5) \oplus \mathcal{P}(7) \\
& \mathcal{R}^{(2)}(0,1)_{5} \otimes_{f} \mathcal{P}(0)=2 \mathcal{P}\left(\frac{10}{3}\right) \oplus 2 \mathcal{P}\left(\frac{1}{3}\right) \oplus \mathcal{P}(1) \oplus \mathcal{P}(2) \\
& \mathcal{R}^{(2)}(1,5) \otimes_{f} \mathcal{P}(0)=2 \mathcal{P}\left(\frac{10}{3}\right) \oplus 2 \mathcal{P}\left(\frac{1}{3}\right) \oplus \mathcal{P}(5) \oplus \mathcal{P}(7) \\
& \mathcal{R}^{(2)}(0,2)_{5} \otimes_{f} \mathcal{P}(0)=2 \mathcal{P}\left(\frac{10}{3}\right) \oplus \mathcal{P}(1) \oplus \mathcal{P}(2) \\
& \mathcal{R}^{(2)}(2,5) \otimes_{f} \mathcal{P}(0)=2 \mathcal{P}\left(\frac{10}{3}\right) \oplus \mathcal{P}(5) \oplus \mathcal{P}(7) \\
& \mathcal{R}^{(2)}(0,1)_{7} \otimes_{f} \mathcal{P}(0)=2 \mathcal{P}\left(\frac{1}{3}\right) \oplus \mathcal{P}(1) \oplus \mathcal{P}(2) \\
& \mathcal{R}^{(2)}(1,7) \otimes_{f} \mathcal{P}(0)=2 \mathcal{P}\left(\frac{1}{3}\right) \oplus \mathcal{P}(5) \oplus \mathcal{P}(7) \\
& \mathcal{P}\left(\frac{1}{3}\right) \otimes_{f} \mathcal{P}(0)=2 \mathcal{P}\left(\frac{10}{3}\right) \oplus 2 \mathcal{P}\left(\frac{1}{3}\right) \oplus 2 \mathcal{P}(1) \oplus 2 \mathcal{P}(5) \\
& \mathcal{P}\left(\frac{10}{3}\right) \otimes_{f} \mathcal{P}(0)=2 \mathcal{P}\left(\frac{10}{3}\right) \oplus 2 \mathcal{P}\left(\frac{1}{3}\right) \oplus 2 \mathcal{P}(1) \oplus 2 \mathcal{P}(5) \\
& \mathcal{P}\left(\frac{5}{8}\right) \otimes_{f} \mathcal{P}(0)=2 \mathcal{P}\left(\frac{1}{8}\right) \oplus 2 \mathcal{P}\left(\frac{33}{8}\right) \oplus 2 \mathcal{P}\left(\frac{21}{8}\right) \oplus 2 \mathcal{P}\left(\frac{5}{8}\right) \oplus 4 \mathcal{W}\left(\frac{-1}{24}\right) \oplus 4 \mathcal{W}\left(\frac{35}{24}\right) \\
& \mathcal{P}\left(\frac{21}{8}\right) \otimes_{f} \mathcal{P}(0)=2 \mathcal{P}\left(\frac{1}{8}\right) \oplus 2 \mathcal{P}\left(\frac{33}{8}\right) \oplus 2 \mathcal{P}\left(\frac{21}{8}\right) \oplus 2 \mathcal{P}\left(\frac{5}{8}\right) \oplus 4 \mathcal{W}\left(\frac{-1}{24}\right) \oplus 4 \mathcal{W}\left(\frac{35}{24}\right) \\
& \mathcal{P}\left(\frac{1}{8}\right) \otimes_{f} \mathcal{P}(0)=2 \mathcal{P}\left(\frac{1}{8}\right) \oplus 2 \mathcal{P}\left(\frac{33}{8}\right) \oplus 2 \mathcal{P}\left(\frac{21}{8}\right) \oplus 2 \mathcal{P}\left(\frac{5}{8}\right) \oplus 4 \mathcal{W}\left(\frac{-1}{24}\right) \oplus 4 \mathcal{W}\left(\frac{35}{24}\right) \\
& \mathcal{P}\left(\frac{33}{8}\right) \otimes_{f} \mathcal{P}(0)=2 \mathcal{P}\left(\frac{1}{8}\right) \oplus 2 \mathcal{P}\left(\frac{33}{8}\right) \oplus 2 \mathcal{P}\left(\frac{21}{8}\right) \oplus 2 \mathcal{P}\left(\frac{5}{8}\right) \oplus 4 \mathcal{W}\left(\frac{-1}{24}\right) \oplus 4 \mathcal{W}\left(\frac{35}{24}\right) \\
& \mathcal{P}(1) \otimes_{f} \mathcal{P}(0)=4 \mathcal{P}\left(\frac{10}{3}\right) \oplus 4 \mathcal{P}\left(\frac{1}{3}\right) \oplus 2 \mathcal{P}(1) \oplus 2 \mathcal{P}(2) \oplus 2 \mathcal{P}(5) \oplus 2 \mathcal{P}(7)
\end{aligned}
$$




$$
\begin{aligned}
& \mathcal{P}(2) \otimes_{f} \mathcal{P}(0)=4 \mathcal{P}\left(\frac{10}{3}\right) \oplus 4 \mathcal{P}\left(\frac{1}{3}\right) \oplus 2 \mathcal{P}(1) \oplus 2 \mathcal{P}(2) \oplus 2 \mathcal{P}(5) \oplus 2 \mathcal{P}(7) \\
& \mathcal{P}(5) \otimes_{f} \mathcal{P}(0)=4 \mathcal{P}\left(\frac{10}{3}\right) \oplus 4 \mathcal{P}\left(\frac{1}{3}\right) \oplus 2 \mathcal{P}(1) \oplus 2 \mathcal{P}(2) \oplus 2 \mathcal{P}(5) \oplus 2 \mathcal{P}(7) \\
& \mathcal{P}(7) \otimes_{f} \mathcal{P}(0)=4 \mathcal{P}\left(\frac{10}{3}\right) \oplus 4 \mathcal{P}\left(\frac{1}{3}\right) \oplus 2 \mathcal{P}(1) \oplus 2 \mathcal{P}(2) \oplus 2 \mathcal{P}(5) \oplus 2 \mathcal{P}(7) \\
& \mathcal{W} \otimes_{f} \mathcal{P}(0)=\mathcal{P}(0) \\
& \mathcal{Q} \otimes_{f} \mathcal{P}(0)=\mathcal{P}(0) \oplus \mathcal{P}\left(\frac{1}{3}\right)
\end{aligned}
$$

This analysis also leads to a consistency check of the assumption that $\mathcal{W}(0)$ has a projective cover. We have arrived at the above list without making use of associativity of the fusion rules. However, it turns out that (B.5) and (B.13) extend the fusion rules in [41, appendix A.4] in an associative manner.

$\mathcal{P}(0)$ cannot have a dual. Unfortunately, the fusion rules of $\mathcal{P}(0)$ with itself cannot be determined by this approach. Indeed, as we shall show in the following, $\mathcal{P}(0) \otimes_{f} \mathcal{P}(0)$ cannot be isomorphic to a direct sum of projectives. Because of lemma appendix B.1 this therefore implies that $\mathcal{P}(0)$ does not have a dual.

Suppose that $\mathcal{P}(0) \otimes_{f} \mathcal{P}(0) \cong \bigoplus_{h} n_{h} \mathcal{P}(h)$, where the sum runs over the 13 values that $h$ takes for the irreducible representations $\mathcal{W}(h)$ according to (1.4). Then

$$
n_{0}=\left\langle\mathcal{P}(0) \otimes_{f} \mathcal{P}(0), \mathcal{W}(0)\right\rangle=\left\langle\mathcal{P}(0) \otimes_{f} \mathcal{P}(0) \otimes_{f} \mathcal{W}(0), \mathcal{W}^{*}\right\rangle=1,
$$

by (B.2) and (B.5), and similarly, using also (B.13),

$$
\begin{aligned}
& \left\langle\mathcal{P}(0) \otimes_{f} \mathcal{P}(0), \mathcal{R}^{(2)}(0,2)_{7}\right\rangle=\left\langle\mathcal{P}(0) \otimes_{f} \mathcal{P}(0) \otimes_{f} \mathcal{R}^{(2)}(0,2)_{7}, \mathcal{W}^{*}\right\rangle \\
& =\left\langle 4 \mathcal{P}(1) \oplus 4 \mathcal{P}(2) \oplus 4 \mathcal{P}(5) \oplus 4 \mathcal{P}(7) \oplus 8 \mathcal{P}\left(\frac{1}{3}\right) \oplus 8 \mathcal{P}\left(\frac{1}{3}\right), \mathcal{W}^{*}\right\rangle=4 .
\end{aligned}
$$

On the other hand,

$$
\left\langle\mathcal{P}(0) \otimes_{f} \mathcal{P}(0), \mathcal{R}^{(2)}(0,2)_{7}\right\rangle=2 n_{2}+2 n_{7}+1,
$$

which is always odd. Thus, $\mathcal{P}(0) \otimes_{f} \mathcal{P}(0)$ cannot be isomorphic to a direct sum of projectives.

\section{B.2. Composition series}

For any representation $R$, we have

$$
\langle\mathcal{P}(h), R\rangle=\left\langle\mathcal{P}(h), R^{*}\right\rangle
$$

since $R$ and $R^{*}$ have the same composition factors. Thus, it follows that

$$
\langle\mathcal{P}(h), \mathcal{P}(0)\rangle=\left\langle\mathcal{P}(h), \mathcal{P}(0)^{*}\right\rangle=\left\langle\mathcal{P}(0), \mathcal{P}(h)^{*}\right\rangle=\langle\mathcal{P}(0), \mathcal{P}(h)\rangle .
$$

We deduce that the composition series of $\mathcal{P}(0)$ only contains $\mathcal{W}(h)$ with $h \in\{0,1,2,5,7\}$ and for $h \in\{1,2,5,7\}$, the multiplicity is 2 .

Now consider the composition series of $\mathcal{P}(h)$ for $h=1,2$ as given in appendix A.1. Let us denote by $N_{h}$ the representation generated by $\mathcal{W}(0)$ at level 1 in $\mathcal{P}(h)$; for the two cases $h=1,2$, it has the composition series

$N_{2}: \mathcal{W}(0) \rightarrow a \mathcal{W}(1) \oplus b \mathcal{W}(2) \rightarrow \mathcal{W}(0) \oplus 2 \mathcal{W}(5) \oplus 2 \mathcal{W}(7) \rightarrow \mathcal{W}(2)$
$N_{1}: \mathcal{W}(0) \rightarrow c \mathcal{W}(1) \oplus d \mathcal{W}(2) \rightarrow \mathcal{W}(0) \oplus 2 \mathcal{W}(5) \oplus 2 \mathcal{W}(7) \rightarrow \mathcal{W}(1)$

for some $a, b, c, d \in \mathbb{Z}_{\geqslant 0}$. Independent of $a, b, c, d, N_{1}$ and $N_{2}$ have $\mathcal{W}(1)$ and $\mathcal{W}(2)$ at level 3 . Thus, $\mathcal{P}(0)$ can at most have one copy of $\mathcal{W}(1)$ and $\mathcal{W}(2)$ at level 1 . Thus, $a=b=c=d=1$ (as they have to be at least 1 ). But then $\mathcal{W}(0)$ in $N_{2}$ gets mapped to a linear combination of two of the $\mathcal{W}(1)$ at level 2 in $\mathcal{P}(2)$. Since each generates a different set of $\mathcal{W}(5)$ and $\mathcal{W}(7)$ at level 3, these have to appear with multiplicity 2 . This fixes the composition series of $\mathcal{P}(0)$ up 
to the appearances of $\mathcal{W}(0)$. Since $\mathcal{W}(0)$ does not allow non-trivial extensions by itself, the most general ansatz is

$\mathcal{W}(0) \rightarrow \mathcal{W}(1) \oplus \mathcal{W}(2) \rightarrow m \mathcal{W}(0) \oplus 2 \mathcal{W}(5) \oplus 2 \mathcal{W}(7) \rightarrow \mathcal{W}(1) \oplus \mathcal{W}(2) \rightarrow n \mathcal{W}(0)$

for some $m \geqslant 1$ and $n \geqslant 0$. In order to have maps $\mathcal{P}(0) \rightarrow N_{h}$ with $h=1,2$, we need $m=1$. Since $\mathcal{R}^{(2)}(0,2)_{7}$ is self-dual, we have

$\left\langle\mathcal{R}^{(2)}(0,2)_{7}, \mathcal{P}(0)\right\rangle=\left\langle\mathcal{W}, \mathcal{P}(0) \otimes_{f} \mathcal{R}^{(2)}(0,2)_{7}\right\rangle=\langle\mathcal{W}, \mathcal{P}(1) \oplus \mathcal{P}(2)\rangle=1$.

Hence $\mathcal{W}(2)$ at level 3 of $\mathcal{P}(0)$ can be joined to at most one $\mathcal{W}(0)$. Repeating the argument with $\mathcal{R}^{(2)}(0,1)_{7}$ gives the same result for $\mathcal{W}(1)$ at level 3 . Thus, $n \in\{0,1,2\}$. But $n=2$ is inconsistent with $n+m+1=\langle\mathcal{P}(0), \mathcal{P}(0)\rangle=\left\langle\mathcal{P}(0)^{*}, \mathcal{P}(0)^{*}\right\rangle=3$ (the identity, and mapping each of the top $\mathcal{W}(0)$ to the bottom $\mathcal{W}(0))$. The remaining two possibilities for the composition series of $\mathcal{P}(0)$ are therefore

$$
\begin{array}{ll}
\text { (a): } & \mathcal{W}(0) \rightarrow \mathcal{W}(1) \oplus \mathcal{W}(2) \rightarrow \mathcal{W}(0) \oplus 2 \mathcal{W}(5) \oplus 2 \mathcal{W}(7) \rightarrow \mathcal{W}(1) \oplus \mathcal{W}(2) \\
\text { (b): } & \mathcal{W}(0) \rightarrow \mathcal{W}(1) \oplus \mathcal{W}(2) \rightarrow \mathcal{W}(0) \oplus 2 \mathcal{W}(5) \oplus 2 \mathcal{W}(7) \rightarrow \mathcal{W}(1) \oplus \mathcal{W}(2) \rightarrow \mathcal{W}(0) .
\end{array}
$$

Very recently, in [46], Zhu's algebra was determined for the $\mathcal{W}_{2,3}$-model. It was found (see proposition 5.1) that Zhu's algebra possesses a representation for which $L_{0}$ has a Jordan block of rank 3 at $h=0$. Because of Zhu's theorem [64] this implies that $\mathcal{W}_{2,3}$ has a highest weight representation with the same property. Since the projective covers have surjections to all highest weight representations, $\mathcal{P}(0)$ must then have a Jordan block of at least this size. As a consequence, we deduce that the composition series (b) is correct, while (a) does not satisfy this condition.

We should mention in passing that the other findings of [46] also fit perfectly with our conjectured embedding diagrams for the other projective covers $\mathcal{P}(h)$. In particular, according to [46] there are rank 2 Jordan blocks for $h=1,2, \frac{1}{8}, \frac{5}{8}, \frac{1}{3}$, while for the remaining values of $h$ (namely $h=5,7, \frac{33}{8}, \frac{21}{8}, \frac{10}{3}$ as well as $h=\frac{-1}{24}, \frac{35}{24}$ ) there are no Jordan blocks. On the face of it this seems to disagree with our proposed structure for say $\mathcal{P}(1)$, since one would guess that the top ' 1 ' should be part of a Jordan block of rank 3. However, one has to recall that for the analysis of Zhu's algebra, only the proper highest weight states contribute, i.e. those states that are annihilated by all positive modes; see for example [65] for a physicist's explanation of this. Given our embedding diagram of $\mathcal{P}(1)$, it is clear that the top ' 1 ' of $\mathcal{P}(1)$ is not highest weight since there is an arrow to the ' 0 ' at level 1 . On the other hand, of the two ' 1 's at level 2 , there will be one linear combination that will not map to the ' 0 ' at level 3 , and that is thus highest weight. It follows that on the level of highest weight states $\mathcal{P}(1)$ only involves a Jordan block of rank 2 for $h=1$, in perfect agreement with the analysis of [46]. The other cases work similarly.

\section{References}

[1] Gurarie V 1993 Logarithmic operators in conformal field theory Nucl. Phys. B 410535 (arXiv:hep-th/9303160)

[2] Cardy J L 1999 Logarithmic correlations in quenched random magnets and polymers arXiv:cond-math/9911024

[3] Gurarie V and Ludwig A W W 2002 Conformal algebras of 2D disordered systems J. Phys. A: Math. Gen. 35 L377 (arXiv:cond-mat/9911392)

[4] Cardy J L 1992 Critical percolation in finite geometries J. Phys. A: Math. Gen. 25 L201 (arXiv:hep-th/9111026)

[5] Watts G M T 1996 A crossing probability for critical percolation in two dimensions J. Phys. A: Math. Gen. 29 L363 (arXiv:cond-mat/9603167)

[6] Flohr M A I and Müller-Lohmann A 2005 Conformal field theory properties of two-dimensional percolation J. Stat. Mech. P12004 (arXiv:hep-th/0507211) 
[7] Rasmussen J and Pearce P A 2007 Fusion algebra of critical percolation J. Stat. Mech. P09002 (arXiv:0706.2716 [hep-th])

[8] Mathieu P and Ridout D 2007 From percolation to logarithmic conformal field theory Phys. Lett. B 657120 (arXiv:0708.0802 [hep-th])

[9] Rasmussen J and Pearce P A $2008 \mathrm{~W}$-extended fusion algebra of critical percolation J. Phys. A: Math. Theor. 41295208 (arXiv:0804.4335 [hep-th])

[10] Ridout D 2009 On the percolation BCFT and the crossing probability of Watts Nucl. Phys. B 810503 (arXiv:0808.3530 [hep-th])

[11] Saleur H 1992 Polymers and percolation in two-dimensions and twisted $N=2$ supersymmetry Nucl. Phys. B 382486 (arXiv:hep-th/9111007)

[12] Read N and Saleur H 2001 Exact spectra of conformal supersymmetric nonlinear sigma models in two dimensions Nucl. Phys. B 613409 (arXiv:hep-th/0106124)

[13] Pearce P A and Rasmussen J 2007 Solvable critical dense polymers J. Stat. Mech. P02015 (arXiv:hep-th/0610273)

[14] Dubail J, Jacobsen J L and Saleur H 2010 Conformal field theory at central charge $c=0$ : A measure of the indecomposability (b) parameters Nucl. Phys. B 834399 (arXiv:1001.1151 [math-ph])

[15] Li W, Song W and Strominger A 2008 Chiral gravity in three dimensions J. High Energy Phys. JHEP04(2008)082 (arXiv:0801.4566 [hep-th])

[16] Grumiller D and Johansson N 2009 Consistent boundary conditions for cosmological topologically massive gravity at the chiral point Int. J. Mod. Phys. D 172367 (arXiv:0808.2575 [hep-th])

[17] Maloney A, Song W and Strominger A 2009 Chiral gravity, log gravity and extremal CFT arXiv:0903.4573 [hep-th]

[18] Kausch H G 1991 Extended conformal algebras generated by a multiplet of primary fields Phys. Lett. B 259448

[19] Gaberdiel M R and Kausch H G 1996 A rational logarithmic conformal field theory Phys. Lett. B 386131 (arXiv:hep-th/9606050)

[20] Gaberdiel M R and Kausch H G 1999 A local logarithmic conformal field theory Nucl. Phys. B 538631 (arXiv:hep-th/9807091)

[21] Kawai S and Wheater J F 2001 Modular transformation and boundary states in logarithmic conformal field theory Phys. Lett. B 508203 (arXiv:hep-th/0103197)

[22] Gaberdiel M R and Runkel I 2006 The logarithmic triplet theory with boundary J. Phys. A: Math. Gen. 3914745 (arXiv:hep-th/0608184)

[23] Rozansky L and Saleur H 1992 Quantum field theory for the multivariable Alexander-Conway polynomial Nucl. Phys. B 376461

[24] Zirnbauer M R 1999 Conformal field theory of the integer quantum Hall plateau transition arXiv:hep-th/9905054

[25] Schomerus V and Saleur H 2006 The Gl(11) WZW model: from supergeometry to logarithmic CFT Nucl. Phys. B 734221 (arXiv:hep-th/0510032)

[26] Götz G, Quella T and Schomerus V 2007 The WZNW model on psu(1,1|2) J. High Energy Phys. JHEP03(2007)003 (arXiv:hep-th/0610070)

[27] Saleur H and Schomerus V 2007 On the SU(2|1) WZNW model and its statistical mechanics applications Nucl. Phys. B 775312 (arXiv:hep-th/0611147)

[28] Quella T and Schomerus V 2007 Free fermion resolution of supergroup WZNW models J. High Energy Phys. JHEP09(2007)085 (arXiv:0706.0744 [hep-th])

[29] Gaberdiel M R 2001 Fusion rules and logarithmic representations of a WZW model at fractional level Nucl. Phys. B 618407 (arXiv:hep-th/0105046)

[30] Lesage F, Mathieu P, Rasmussen J and Saleur H 2002 The $\widehat{s u}(2)_{-1 / 2}$ WZW model and the $\beta \gamma$ system Nucl. Phys. B 647363 (arXiv:hep-th/0207201)

[31] Lesage F, Mathieu P, Rasmussen J and Saleur H 2004 Logarithmic lift of the $\widehat{s u}(2)_{-1 / 2}$ model Nucl. Phys. B 686313 (arXiv:hep-th/0311039)

[32] Ridout D 2010 sl(2) $-1 / 2$ and the triplet model Nucl. Phys. B 835314 (arXiv:1001.3960 [hep-th])

[33] Pearce P A, Rasmussen J and Zuber J-B 2006 Logarithmic minimal models J. Stat. Mech. P11017 (arXiv:hep-th/0607232)

[34] Kytölä K and Ridout D 2009 On staggered indecomposable Virasoro modules J. Math. Phys. 50123503 (arXiv:0905.0108 [math-ph])

[35] Feigin B L, Gainutdinov A M, Semikhatov A M and Tipunin I Y 2006 Logarithmic extensions of minimal models: Characters and modular transformations Nucl. Phys. B 757303 (arXiv:hep-th/0606196)

[36] Eberle H and Flohr M 2006 Virasoro representations and fusion for general augmented minimal models J. Phys. A: Math. Gen. 3915245 (arXiv:hep-th/0604097) 
[37] Feigin B L, Gainutdinov A M, Semikhatov A M and Tipunin I Y 2007 Kazhdan-Lusztig-dual quantum group for logarithmic extensions of Virasoro minimal models J. Math. Phys. 48032303 (arXiv:math.QA/0606506)

[38] Semikhatov A M 2007 A note on the logarithmic $\left(p, p^{\prime}\right)$ fusion arXiv:0710.5157 [hep-th]

[39] Rasmussen J 2009 W-extended logarithmic minimal models Nucl. Phys. B 807495 (arXiv:0805.2991 [hep-th])

[40] Rasmussen J 2009 Polynomial fusion rings of W-extended logarithmic minimal models J. Math. Phys. 50043512 (arXiv:0812.1070 [hep-th])

[41] Gaberdiel M R, Runkel I and Wood S 2009 Fusion rules and boundary conditions in the $c=0$ triplet model J. Phys. A: Math. Theor. 42325403 (arXiv:0905.0916 [hep-th])

[42] Rasmussen J 2010 Fusion of irreducible modules in WLM(p,p') J. Phys. A: Math. Theor. 43045210 (arXiv:0906.5414 [hep-th])

[43] Wood S 2010 Fusion rules of the $\mathcal{W}_{p, q}$ triplet models J. Phys. A: Math. Theor. 43045212 (arXiv:0907.4421 [hep-th])

[44] Adamovic D and Milas A 2009 On W-algebras associated to (2,p) minimal models and their representations arXiv:0908.4053 [math.QA]

[45] Rasmussen J 2010 Graph fusion algebras of WLM(p,p') Nucl. Phys. B 830493 (arXiv:0911.3229 [hep-th])

[46] Adamovic D and Milas A 2010 The structure of Zhu's algebras for certain W-algebras arXiv:1006.5134 [math.QA]

[47] Runkel I 1999 Boundary structure constants for the A-series Virasoro minimal models Nucl. Phys. B 549563 (arXiv:hep-th/9811178)

[48] Felder G, Fröhlich J, Fuchs J and Schweigert C 2002 Correlation functions and boundary conditions in RCFT and three-dimensional topology Compos. Math. 131189 (arXiv:hep-th/9912239)

[49] Fuchs J, Runkel I and Schweigert C 2002 TFT construction of RCFT correlators: 1. Partition functions Nucl. Phys. B 646353 (arXiv:hep-th/0204148)

[50] Petkova V B and Zuber J-B 2001 The many faces of Ocneanu cells Nucl. Phys. B 603449 (arXiv:hep-th/0101151)

[51] Fjelstad J, Fuchs J, Runkel I and Schweigert C 2008 Uniqueness of open/closed rational CFT with given algebra of open states Adv. Theor. Math. Phys. 121283 (arXiv:hep-th/0612306)

[52] Kong L and Runkel I 2009 Cardy algebras and sewing constraints: 1 Commun. Math. Phys. 292871 (arXiv:0807.3356 [math.QA])

[53] Gaberdiel M R and Runkel I 2008 From boundary to bulk in logarithmic CFT J. Phys. A: Math. Theor. 41075402 (arXiv:0707.0388 [hep-th])

[54] Huang Y Z, Lepowsky J and Zhang L 2007 Logarithmic tensor product theory for generalized modules for a conformal vertex algebra arXiv:0710.2687 [math.QA]

[55] Huang Y-Z 2009 Cofiniteness conditions, projective covers and the logarithmic tensor product theory $J$. Pure Appl. Algebra 213458 (arXiv:0712.4109 [math.QA])

[56] Huang Y-Z 2009 Representations of vertex operator algebras and braided finite tensor categories arXiv:0903.4233 [math.QA]

[57] Cardy J L 1989 Boundary conditions, fusion rules and the Verlinde formula Nucl. Phys. B 324581

[58] Joyal A and Street R 1993 Braided tensor categories Adv. Math. 10220

[59] Cardy J L and Lewellen D C 1991 Bulk and boundary operators in conformal field theory Phys. Lett. B 259274

[60] Lewellen D C 1992 Sewing constraints for conformal field theories on surfaces with boundaries Nucl. Phys. B 372654

[61] Kong L 2008 Open-closed field algebras Commun. Math. Phys. 280207 (arXiv:math.QA/0610293)

[62] Wood S, in preparation

[63] Nagatomo K and Tsuchiya A 2009 The triplet vertex operator algebra W(p) and the restricted quantum group at root of unity arXiv:0902.4607 [math.QA]

[64] Zhu Y 1996 Modular invariance of characters of vertex operator algebras J. Am. Math. Soc. 9 237-302

[65] Gaberdiel M R and Goddard P 2000 Axiomatic conformal field theory Commun. Math. Phys. 209549 (arXiv:hep-th/9810019)

[66] Bakalov B and Kirillov A A 2001 Lectures on Tensor Categories and Modular Functors (Providence, RI: American Mathematical Society)

[67] Kazhdan D and Lusztig G 1994 Tensor structures arising from affine Lie algebras: IV J. Am. Math. Soc. 7383 